\title{
Integral Geometric Tools for Stochastic Geometry
}

\author{
Rolf Schneider
}

Mathematisches Institut

Albert-Ludwigs-Universität, D-79104 Freiburg i. Br., Germany

rolf.schneider@math. uni-freiburg.de

\section{Introduction}

Integral geometry deals with measures on sets of geometric objects, and in particular with the determination of the total measure of various such sets having geometric significance. For example, given two convex bodies in Euclidean space, what is the total invariant measure of the set of all rigid motions which bring the first set into a position where it has nonempty intersection with the second one? Or, what is the total invariant measure of the set of all planes of a fixed dimension having nonempty intersection with a given convex body? Both questions have classical answers, known as the kinematic formula and the Crofton formula, respectively. Results of this type are useful in stochastic geometry. Basic random closed sets, the stationary and isotropic Boolean models with convex grains, are obtained by taking union sets of certain stochastic processes of convex bodies. Simple numerical parameters for the description of such Boolean models are functional densities related to the specific volume, surface area, or Euler characteristic. Kinematic formulae are indispensable tools for the investigation and estimation of such parameters. Weakening the hypotheses on Boolean models, requiring less invariance properties and admitting more general set classes, necessitates the generalization of integral geometric formulae in various directions. An introduction to the needed basic formulae and a discussion of their extensions, analogues and ramifications is the main purpose of the following. The section headings are:

1. From Hitting Probabilities to Kinematic Formulae

2. Localizations and Extensions

3. Translative Integral Geometry

4. Intersection Formulae Without Invariance

A simplifying aspect of our selection from the realm of integral geometry is, on the side of geometric operations, the restriction to intersections of fixed and variable sets and, on the side of measures, the restriction to Haar measures on 
groups of rigid motions or translations; only on spaces of flats do we consider non-invariant measures as well.

The following is meant as an introduction to the integral geometry that is relevant for stochastic geometry, with a few glimpses to more recent developments of independent interest. The character of the presentation varies from introductory to survey parts, and corresponding to this, proofs of the stated results are sometimes given in full, occasionally sketched, and often omitted.

\section{From Hitting Probabilities to Kinematic Formulae}

This chapter gives an introduction to the classical kinematic and Crofton formulae for convex bodies. We start with a deliberately vague question on certain hitting probabilities. This leads us in a natural way to the necessity of calculating certain kinematic measures, as well as to the embryonic idea of a Boolean model.

\subsection{A Heuristic Question on Hitting Probabilities}

The following question was posed and treated in [4]. Let $K$ and $L$ be two given convex bodies (nonempty compact convex sets) in Euclidean space $\mathbb{R}^{n}$. We use $K$ to generate a random field of congruent copies of $K$. That means, countably many congruent copies of $K$ are laid out randomly and independently in space. The bodies may overlap. It is assumed that the random system has a well defined number density, that is, an expected mean number of particles per unit volume. The body $L$ is used as a fixed test body. For a given number $j \in \mathbb{N}_{0}$, we ask for the probability, $p_{j}$, of the event that the test body $L$ is hit by (that is, has nonempty intersection with) exactly $j$ bodies of the random field.

So far, of course, this is only an imprecise heuristic question. It will require several steps to make the question precise. In a first step, we choose a large ball $B_{r}$, of radius $r$ and origin 0 , that contains $L$, and we consider only one randomly moving congruent copy of $K$, under the condition that it hits $B_{r}$. What is the probability that it hits also $L$ ? To make this a meaningful question, we have to specify the probability distribution of the randomly moving body. The geometrically most natural assumption is that this distribution be induced from the motion invariant measure $\mu$ on the group $G_{n}$ of rigid motions of $\mathbb{R}^{n}$. This means that we represent the congruent copies of $K$ in the form $g K$, where $g \in G_{n}$ is a rigid motion. We define a probability distribution $\mathbb{P}$ on the space $\mathcal{K}$ of convex bodies (equipped with the Hausdorff metric) in $\mathbb{R}^{n}$ by

$$
\mathbb{P}(g K \in \mathcal{A})=\frac{\mu\left(\left\{g \in G_{n}: g K \cap B_{r} \neq \emptyset, g K \in \mathcal{A}\right\}\right)}{\mu\left(\left\{g \in G_{n}: g K \cap B_{r} \neq \emptyset\right\}\right)}
$$

for Borel sets $\mathcal{A} \subset \mathcal{K}$. 
Now it makes sense to ask for the probability, $p$, of the event that $g K$ meets the body $L \subset B_{r}$, and this probability is given by

$$
p=\frac{\mu(K, L)}{\mu\left(K, B_{r}\right)},
$$

where we have put

$$
\mu(K, M):=\mu\left(\left\{g \in G_{n}: g K \cap M \neq \emptyset\right\}\right)
$$

for convex bodies $K$ and $M$.

How can we compute $\mu(K, M)$ ? Let us first suppose that $K$ is a ball of radius $\rho$. Then the measure of all motions $g$ that bring $K$ in a hitting position with $M$ is (under suitable normalization) equal to the measure of all translations which bring the centre of $K$ into the parallel body

$$
M+B_{\rho}:=\left\{m+b: m \in M, b \in B_{\rho}\right\},
$$

and hence to the volume of this body. By a fundamental result of convex geometry, this volume is a polynomial of degree at most $n$ in the parameter $\rho$, usually written as

$$
\lambda_{n}\left(M+B_{\rho}\right)=\sum_{i=0}^{n} \rho^{n-i} \kappa_{n-i} V_{i}(M)
$$

( $\lambda_{n}=$ Lebesgue measure in $\mathbb{R}^{n}, \kappa_{j}=$ volume of the $j$-dimensional unit ball). This result, known as the Steiner formula, defines important functionals, the intrinsic volumes $V_{0}, \ldots, V_{n}$.

We see already from this special case, $K=B_{\rho}$, that in the computation of the measure $\mu(K, M)$ the intrinsic volumes must play an essential role. It is a remarkable fact that no further functionals are needed for the general case. The principal kinematic formula of integral geometry, in its specialization to convex bodies, says that

$$
\mu(K, M)=\sum_{i=0}^{n} \alpha_{n i} V_{i}(K) V_{n-i}(M),
$$

with certain explicit constants $\alpha_{n i}$. For the moment, we take this formula for granted. A proof will be given in Subsection 1.4.

Recalling that the probability $p$, of the event that a randomly moving congruent copy of $K$ hitting $B_{r}$ also hits $L$, is given by (1), we have now found that

$$
p=\frac{\sum_{i=0}^{n} \alpha_{n i} V_{i}(K) V_{n-i}(L)}{\sum_{i=0}^{n} \alpha_{n i} V_{i}(K) r^{n-i} \kappa_{n}},
$$

which depends (for fixed $r$ ) only on the intrinsic volumes of $K$ and $L$.

In the second step, we consider $m$ randomly chosen congruent copies of $K$, given in the form $g_{1} K, \ldots, g_{m} K$ with random motions $g_{1}, \ldots, g_{m}$. We assume 
that these random copies are stochastically independent and that they all have the same distribution, as described above. For $j \in\{0,1, \ldots, m\}$, let $p_{j}$ denote the probability of the event that the test body $L$ is hit by exactly $j$ of the random congruent copies of $K$. The assumed independence leads to a binomial distribution, thus

$$
p_{j}=\left(\begin{array}{c}
m \\
j
\end{array}\right) p^{j}(1-p)^{m-j}
$$

with $p$ given by (4).

In the third step, we let the radius $r$ of the ball $B_{r}$ and the number $m$ of particles tend to $\infty$, but in such a way that

$$
\lim _{\substack{m \rightarrow \infty \\ r \rightarrow \infty}} \frac{m}{\lambda_{n}\left(B_{r}\right)}=\gamma
$$

with a positive constant $\gamma$. From

$$
m p=\frac{m}{\lambda_{n}\left(B_{r}\right)} \frac{\lambda_{n}\left(B_{r}\right)}{\mu\left(K, B_{r}\right)} \mu(K, L) \quad \text { and } \quad \lim _{r \rightarrow \infty} \frac{\mu\left(K, B_{r}\right)}{\lambda_{n}\left(B_{r}\right)}=1
$$

we get $m p \rightarrow \gamma \mu(K, L)=: \theta$ and hence

$$
\lim _{r \rightarrow \infty} p_{j}=\frac{\theta^{j}}{j !} \mathrm{e}^{-\theta}
$$

with

$$
\theta=\gamma \mu(K, L)=\gamma \sum_{i=0}^{n} \alpha_{n i} V_{i}(K) V_{n-i}(L) .
$$

We have found, not surprisingly, a Poisson distribution. Its parameter, $\theta$, is expressed explicitly in terms of the constant $\gamma$, which can be interpreted as the number density of our random system of convex bodies, and the intrinsic volumes of $K$ and $L$.

This is the answer given in [4]. The answer is explicit and elegant, but still not quite satisfactory. What the authors have computed is a limit of probabilities, and this turned out to be a Poisson distribution. However, this Poisson distribution is not yet interpreted as the distribution of a well-defined random variable. What we would prefer, and what is needed for applications, is a model that allows us to consider from the beginning countably infinite systems of randomly placed convex bodies, with suitable independence properties. This requirement leads us, inevitably and in a natural way, to the notion of a Poisson process of convex particles. More general versions of such particle processes and their union sets, the Boolean models, are one of the topics of Wolfgang Weil's lectures. Our task is now to provide the integral geometry that is needed for a quantitative treatment of Boolean models. We begin with the Steiner and kinematic formulae. 


\subsection{Steiner Formula and Intrinsic Volumes}

Our first aim is to prove the Steiner formula (2) and to use it for introducing the intrinsic volumes, which are basic functionals of convex bodies. First we collect some notation.

On $\mathbb{R}^{n}$, we use the standard scalar product $\langle\cdot, \cdot\rangle$ and the Euclidean norm $\|\cdot\|$. The unit ball of $\mathbb{R}^{n}$ is $B^{n}:=\left\{x \in \mathbb{R}^{n}:\|x\| \leq 1\right\}$, and its boundary is the unit sphere $S^{n-1}$. By $\lambda_{k}$ we denote the $k$-dimensional Lebesgue measure in $k$-dimensional flats (affine subspaces) of $\mathbb{R}^{n}$. For convex bodies $K$ we write $\lambda_{n}(K)=: V_{n}(K)$. The constant $\kappa_{n}=\pi^{n / 2} / \Gamma(1+n / 2)$ gives the volume of the unit ball $B^{n}$. Spherical Lebesgue measure on $S^{k}$ is denoted by $\sigma_{k}$.

The space $\mathcal{K}$ of convex bodies in $\mathbb{R}^{n}$ is equipped with the Hausdorff metric. The sum of convex bodies $K, L \in \mathcal{K}$ is defined by

$$
K+L:=\{x+y: x \in K, y \in L\} .
$$

A special case gives the parallel body of $K$ at distance $\rho \geq 0$,

$$
K_{\rho}:=K+\rho B^{n}=\left\{x \in \mathbb{R}^{n}: d(K, x) \leq \rho\right\},
$$

where

$$
d(K, x):=\min \{\|x-y\|: y \in K\}
$$

is the distance of $x$ from $K$. Let $K \in \mathcal{K}$ be a convex body. For $x \in \mathbb{R}^{n}$, there is a unique point $p(K, x)$ in $K$ nearest to $x$, thus

$$
\|p(K, x)-x\|=\min \{\|y-x\|: y \in K\}=d(K, x) .
$$

This defines a Lipschitz map $p(K, \cdot): \mathbb{R}^{n} \rightarrow K$, which is called the nearestpoint map of $K$, or the metric projection onto $K$.

For the case of a planar convex polygon $P$, the reader will easily convince herself, after drawing a picture of the parallel body $P_{\rho}$, that the area of this parallel body is a quadratic polynomial in $\rho$. The simple idea showing this extends to higher dimensions, as follows.

A polyhedral set in $\mathbb{R}^{n}$ is a set which can be represented as the intersection of finitely many closed halfspaces. A bounded non-empty polyhedral set is called a convex polytope or briefly a polytope. Let $P$ be a polytope. If $H$ is a supporting hyperplane of $P$, then $P \cap H$ is again a polytope. The set $F:=P \cap H$ is called a face of $P$, and an $m$-face if $\operatorname{dim} F=m, m \in\{0, \ldots, n-1\}$. If $\operatorname{dim} P=n$, we consider $P$ as an $n$ face of itself. By $\mathcal{F}_{m}(P)$ we denote the set of all $m$-faces of $P$, and we put $\mathcal{F}(P):=\bigcup_{m=0}^{n} \mathcal{F}_{m}(P)$. For $m \in\{0, \ldots, n-1\}, F \in \mathcal{F}_{m}(P)$, and a point $x \in$ relint $F$ (the relative interior of $F$ ), let $N(P, F)$ be the normal cone of $P$ at $F$; this is the closed convex cone of outer normal vectors of supporting hyperplanes to $P$ at $x$, together with the zero vector. It does not depend upon the choice of $x$. The number 


$$
\gamma(F, P):=\frac{\lambda_{n-m}\left(N(P, F) \cap B^{n}\right)}{\kappa_{n-m}}=\frac{\sigma_{n-m-1}\left(N(P, F) \cap S^{n-1}\right)}{(n-m) \kappa_{n-m}}
$$

is called the external angle of $P$ at its face $F$. We also put $\gamma(P, P)=1$ and $\gamma(F, P)=0$ if either $F=\emptyset$ or $F$ is not a face of $P$.

Now let a polytope $P$ and a number $\rho>0$ be given. For $x \in \mathbb{R}^{n}$, the nearest point $p(P, x)$ lies in the relative interior of a unique face of $P$. Therefore,

$$
P_{\rho}=\bigcup_{F \in \mathcal{F}(P)}\left[P_{\rho} \cap p(P, \cdot)^{-1}(\operatorname{relint} F)\right]
$$

is a disjoint decomposition of the parallel body $P_{\rho}$. For $m \in\{0, \ldots, n-1\}$ and $F \in \mathcal{F}_{m}$ it follows from the properties of the nearest point map that

$$
P_{\rho} \cap p(P, \cdot)^{-1}(\operatorname{relint} F)=\operatorname{relint} F \oplus\left(N(P, F) \cap \rho B^{n}\right),
$$

where $\oplus$ denotes a direct orthogonal sum. An application of Fubini's theorem gives

$$
\begin{aligned}
\lambda_{n}\left(P_{\rho} \cap p(P, \cdot)^{-1}(\operatorname{relint} F)\right) & =\lambda_{m}(F) \lambda_{n-m}\left(N(P, F) \cap \rho B^{n}\right) \\
& =\lambda_{m}(F) \rho^{n-m} \kappa_{n-m} \gamma(F, P) .
\end{aligned}
$$

Hence, if we define

$$
V_{m}(P):=\sum_{F \in \mathcal{F}_{m}(P)} \lambda_{m}(F) \gamma(F, P)
$$

it follows from (6) that

$$
V_{m}\left(P_{\rho}\right):=\sum_{m=0}^{n} \rho^{n-m} \kappa_{n-m} V_{m}(P) .
$$

This can be extended to general convex bodies:

Theorem 1.1 (Steiner formula). There are functionals $V_{m}: \mathcal{K} \rightarrow \mathbb{R}, m=$ $0, \ldots, n$, such that, for $K \in \mathcal{K}$ and $\rho \geq 0$,

$$
V_{n}\left(K_{\rho}\right)=\sum_{m=0}^{n} \rho^{n-m} \kappa_{n-m} V_{m}(K) .
$$

For the proof, we use (9) for $\rho=1, \ldots, n+1$ and solve a system of linear equations with a Vandermonde determinant, to obtain expressions

$$
V_{m}(P)=\sum_{\nu=1}^{n+1} a_{m \nu} V_{n}\left(P_{\nu}\right), \quad m=0, \ldots, n,
$$


with coefficients $a_{m \nu}$ independent of the polytope $P$. With these coefficients, we define

$$
V_{m}(K):=\sum_{\nu=1}^{n+1} a_{m \nu} V_{n}\left(K_{\nu}\right)
$$

for arbitrary convex bodies $K \in \mathcal{K}$. Since, for each fixed $\rho \geq 0$, the mapping $K \mapsto V_{n}\left(K+\rho B^{n}\right)$ is continuous with respect to the Hausdorff metric, the functional $V_{m}$ is continuous. Using this and approximating $K$ by a sequence of polytopes, we obtain the asserted result from (9).

The polynomial expansion (10) is known as the Steiner formula. The functionals $V_{0}, \ldots, V_{n}$, uniquely determined by (10), are called the intrinsic volumes (also, with different normalizations, the quermassintegrals or Minkowski functionals). About their geometric meaning, the following can be said. For polytopes, there are the explicit representations (8). They are particularly simple in the cases $m=n, n-1,0$, and the results carry over to general convex bodies: $V_{n}$ is the volume, $2 V_{n-1}$ is the surface area (the $(n-1)$-dimensional Hausdorff measure of the boundary, for bodies of dimension $n$ ), and $V_{0}$ is the constant 1 . The functional $V_{0}$, although trivial on convex bodies, has its own name and symbol: the Euler characteristic $\chi$; the reason will become clear when we consider an extension of $V_{0}$ to more general sets. Also the other intrinsic volumes have simple interpretations, either differential geometric, under smoothness assumptions (see Subsection 2.1), or integral geometric (see Subsection 1.4, formula (16)).

It is easily seen from the Steiner formula that the map $V_{m}: \mathcal{K} \rightarrow \mathbb{R}$, $m \in\{0, \ldots, n\}$, has the following properties:

- $V_{m}$ is invariant under rigid motions,

- $V_{m}$ is continuous with respect to the Hausdorff metric,

- $V_{m}$ is homogeneous of degree $m$,

that is, it satisfies

$$
V_{m}(\alpha K)=\alpha^{m} V_{m}(K) \quad \text { for } \alpha \geq 0 .
$$

Using (8) (and approximation), one shows without difficulty that

- $V_{m}$ does not depend on the dimension of the surrounding space,

that is, if $K$ lies in a Euclidean subspace of $\mathbb{R}^{n}$, then computation of $V_{m}(K)$ in that subspace leads to the same result as computation of $V_{m}(K)$ in $\mathbb{R}^{n}$. From the integral geometric representation (16) to be proved later it is seen that

- $V_{m}$ is increasing under set inclusion,

which means that $K \subset L$ for convex bodies $K, L$ implies $V_{m}(K) \leq V_{m}(L)$. A very important property is the additivity. A functional $\varphi: \mathcal{K} \rightarrow A$ with values in an abelian group $A$ is called additive or a valuation if 


$$
\varphi(K \cup L)+\varphi(K \cap L)=\varphi(K)+\varphi(L)
$$

whenever $K, L$ are convex bodies such that $K \cup L$ is convex (which implies that $K \cap L$ is not empty). If $\varphi$ is an additive functional, one extends its definition by putting $\varphi(\emptyset):=0$. As announced, we have:

- $V_{m}$ is additive.

For a proof, one shows that, for fixed $\rho \geq 0$, the function $\mathbf{1}_{\rho}$ defined by

$$
\mathbf{1}_{\rho}(K, x):=\left\{\begin{array}{l}
1 \text { if } x \in K_{\rho}, \\
0 \text { if } x \in \mathbb{R}^{n} \backslash K_{\rho}
\end{array}\right.
$$

for $K \in \mathcal{K}$ and $x \in \mathbb{R}^{n}$ satisfies

$$
\mathbf{1}_{\rho}(K \cup L, x)+\mathbf{1}_{\rho}(K \cap L, x)=\mathbf{1}_{\rho}(K, x)+\mathbf{1}_{\rho}(L, x) .
$$

Integration over $x$ with respect to the Lebesgue measure yields

$$
V_{n}\left((K \cup L)_{\rho}\right)+V_{n}\left((K \cap L)_{\rho}\right)=V_{n}\left(K_{\rho}\right)+V_{n}\left(L_{\rho}\right)
$$

for $\rho \geq 0$. Now an application of the Steiner formula and comparison of the coefficients shows that each $V_{m}$ is additive.

Hints to the literature. For the fundamental facts about convex bodies, we refer to [39], where details of the foregoing can be found.

\subsection{Hadwiger's Characterization Theorem for Intrinsic Volumes}

Of the properties established above for the intrinsic volumes, already a suitable selection is sufficient for an axiomatic characterization. This is the content of Hadwiger's celebrated characterization theorem:

Theorem 1.2 (Hadwiger's characterization theorem). Suppose that $\psi$ : $\mathcal{K} \rightarrow \mathbb{R}$ is an additive, continuous, motion invariant function. Then there are constants $c_{0}, \ldots, c_{n}$ so that

$$
\psi(K)=\sum_{i=0}^{n} c_{i} V_{i}(K)
$$

for all $K \in \mathcal{K}$.

This result not only throws light on the importance of the intrinsic volumes, showing that they are essentially the only functionals on convex bodies sharing some very natural geometric properties with the volume, it is also useful. Following Hadwiger, we employ it to prove some integral geometric formulae in an elegant way. Whereas Hadwiger's original proof of his characterization 
theorem was quite long, a shorter proof was published in 1995 by Daniel Klain. It will be presented here, except that a certain extension theorem for additive functionals is postponed to Subsection 2.2 and a certain analytical result will be taken for granted.

The crucial step for a proof of Hadwiger's characterization theorem is the following result.

Proposition 1.1. Suppose that $\psi: \mathcal{K} \rightarrow \mathbb{R}$ is an additive, continuous, motion invariant function satisfying $\psi(K)=0$ whenever either $\operatorname{dim} K<n$ or $K$ is a unit cube. Then $\psi=0$.

Proof. The proof proceeds by induction with respect to the dimension. For $n=0$, there is nothing to prove. If $n=1, \psi$ vanishes on (closed) segments of unit length, hence on segments of length $1 / k$ for $k \in \mathbb{N}$ and therefore on segments of rational length. By continuity, $\psi$ vanishes on all segments and thus on $\mathcal{K}$.

Now let $n>1$ and suppose that the assertion has been proved in dimensions less than $n$. Let $H \subset \mathbb{R}^{n}$ be a hyperplane and $I$ a closed line segment of length 1 , orthogonal to $H$. For convex bodies $K \subset H$ define $\varphi(K):=\psi(K+I)$. Clearly $\varphi$ has, relative to $H$, the properties of $\psi$ in the assertion, hence the induction hypothesis yields $\varphi=0$. For fixed $K \subset H$, we thus have $\psi(K+I)=0$, and a similar argument as used above for $n=1$ shows that $\psi(K+S)=0$ for any closed segment $S$ orthogonal to $H$. Thus $\psi$ vanishes on right convex cylinders.

Let $K \subset H$ be a convex body again, and let $S=\operatorname{conv}\{0, s\}$ be a segment not parallel to $H$. If $m \in \mathbb{N}$ is sufficiently large, the cylinder $Z:=K+$ $m S$ can be cut by a hyperplane $H^{\prime}$ orthogonal to $S$ so that the two closed halfspaces $H^{-}, H^{+}$bounded by $H^{\prime}$ satisfy $K \subset H^{-}$and $K+m s \subset H^{+}$. Then $\bar{Z}:=\left[\left(Z \cap H^{-}\right)+m s\right] \cup\left(Z \cap H^{+}\right)$is a right cylinder, and we deduce that $m \mu(K+S)=\mu(Z)=\mu(\bar{Z})=0$. Thus $\psi$ vanishes on arbitrary convex cylinders.

It can be shown that the continuous additive function $\psi$ on $\mathcal{K}$ satisfies the more general additivity property

$$
\psi\left(\bigcup_{i=1}^{k} K_{i}\right)=\sum_{i=1}^{k} \psi\left(K_{i}\right)
$$

whenever $K_{1}, \ldots, K_{k}$ are convex bodies such that $\operatorname{dim}\left(K_{i} \cap K_{j}\right)<n$ for $i \neq j$ and that $\bigcup_{i=1}^{k} K_{i}$ is convex. This follows from Theorem 2.2 and (35) below and the fact that $\psi$ has been assumed to vanish on convex bodies of dimension less than $n$.

Let $P$ be a polytope and $S$ a segment. The sum $P+S$ has a decomposition

$$
P+S=\bigcup_{i=1}^{k} P_{i},
$$


where $P_{1}=P$, the polytope $P_{i}$ is a convex cylinder for $i>1$, and $\operatorname{dim}\left(P_{i} \cap\right.$ $\left.P_{j}\right)<n$ for $i \neq j$. It follows that $\psi(P+S)=\psi(P)$. By induction, we obtain $\psi(P+Z)=\psi(P)$ if $Z$ is a finite sum of segments. Such a body $Z$ is called a zonotope, and a convex body which can be approximated by zonotopes is called a zonoid. Since the function $\psi$ is continuous, it follows that $\psi(K+Z)=\psi(K)$ for arbitrary convex bodies $K$ and zonoids $Z$.

We have to use an analytic result for which we do not give a proof. Let $K$ be a centrally symmetric convex body which is sufficiently smooth (say, its support function is of class $C^{\infty}$ ). Then there exist zonoids $Z_{1}, Z_{2}$ so that $K+Z_{1}=Z_{2}$ (this can be seen from Section 3.5 in the book [39], especially Theorem 3.5.3). We conclude that $\psi(K)=\psi\left(K+Z_{1}\right)=\psi\left(Z_{2}\right)=0$. Since every centrally symmetric convex body $K$ can be approximated by bodies which are centrally symmetric and sufficiently smooth, it follows from the continuity of $\psi$ that $\psi(K)=0$ for all centrally symmetric convex bodies.

Now let $\Delta$ be a simplex, say $\Delta=\operatorname{conv}\left\{0, v_{1}, \ldots, v_{n}\right\}$, without loss of generality. Let $v:=v_{1}+\cdots+v_{n}$ and $\Delta^{\prime}:=\operatorname{conv}\left\{v, v-v_{1}, \ldots, v-v_{n}\right\}$, then $\Delta^{\prime}=-\Delta+v$. The vectors $v_{1}, \ldots, v_{n}$ span a parallelotope $P$. It is the union of $\Delta, \Delta^{\prime}$ and the part of $P$, denoted by $Q$, that lies between the hyperplanes spanned by $v_{1}, \ldots, v_{n}$ and $v-v_{1}, \ldots, v-v_{n}$, respectively. Now $Q$ is a centrally symmetric polytope, and $\Delta \cap Q, \Delta^{\prime} \cap Q$ are of dimension $n-1$. We deduce that $0=\psi(P)=\psi(\Delta)+\psi(Q)+\psi\left(\Delta^{\prime}\right)$, thus $\psi(-\Delta)=-\psi(\Delta)$. If the dimension $n$ is even, then $-\Delta$ is obtained from $\Delta$ by a proper rigid motion, and the motion invariance of $\psi$ yields $\psi(\Delta)=0$. If the dimension $n>1$ is odd, we decompose $\Delta$ as follows. Let $z$ be the centre of the inscribed ball of $\Delta$, and let $p_{i}$ be the point where this ball touches the facet $F_{i}$ of $\Delta(i=1, \ldots, n+1)$. For $i \neq j$, let $Q_{i j}$ be the convex hull of the face $F_{i} \cap F_{j}$ and the points $z, p_{i}, p_{j}$. The polytope $Q_{i j}$ is invariant under reflection in the hyperplane spanned by $F_{i} \cap F_{j}$ and $z$. If $Q_{1}, \ldots, Q_{m}$ are the polytopes $Q_{i j}$ for $1 \leq i<j \leq n+1$ in any order, then $P=\bigcup_{r=1}^{m} Q_{r}$ and $\operatorname{dim}\left(Q_{r} \cap Q_{s}\right)<n$ for $r \neq s$. Since $-Q_{r}$ is the image of $Q_{r}$ under a proper rigid motion, we have $\psi(-\Delta)=\sum \psi\left(-Q_{r}\right)=$ $\sum \psi\left(Q_{r}\right)=\psi(\Delta)$. Thus $\psi(\Delta)=0$ for every simplex $\Delta$.

Decomposing a polytope $P$ into simplices, we obtain $\psi(P)=0$. The continuity of $\psi$ now implies $\psi(K)=0$ for all convex bodies $K$. This finishes the induction and hence the proof of the proposition.

Proof of Theorem 1.2. We use induction on the dimension. For $n=0$, the assertion is trivial. Suppose that $n>0$ and the assertion has been proved in dimensions less than $n$. Let $H \subset \mathbb{R}^{n}$ be a hyperplane. The restriction of $\psi$ to the convex bodies lying in $H$ is additive, continuous and invariant under motions of $H$ into itself. By the induction hypothesis, there are constants $c_{0}, \ldots, c_{n-1}$ so that $\psi(K)=\sum_{i=0}^{n-1} c_{i} V_{i}(K)$ holds for convex bodies $K \subset$ $H$ (here we use the fact that the intrinsic volumes do not depend on the dimension of the surrounding space). By the motion invariance of $\psi$ and $V_{i}$, this holds for all $K \in \mathcal{K}$ of dimension less than $n$. It follows that the function $\psi^{\prime}$ defined by 


$$
\psi^{\prime}(K):=\psi(K)-\sum_{i=0}^{n} c_{i} V_{i}(K)
$$

for $K \in \mathcal{K}$, where $c_{n}$ is chosen so that $\psi^{\prime}$ vanishes at a fixed unit cube, satisfies the assumptions of Theorem 1.1. Hence $\psi^{\prime}=0$, which completes the proof of Theorem 2.

Hints to the literature. Hadwiger's characterization theorem was first proved for dimension three in [13] and for general dimension in [14]; the proof is reproduced in [16]. The simpler proof as presented here appears in [24]; see also $[25]$.

\subsection{Integral Geometric Formulae}

We use Hadwiger's characterization theorem to prove some basic integral geometric results for convex bodies. They involve invariant measures on groups of motions or on spaces of flats, where these groups and homogeneous spaces are equipped with their usual topologies. In the following, a measure on a topological space $X$ is always defined on $\mathcal{B}(X)$, its $\sigma$-algebra of Borel sets. Let $S O_{n}$ be the group of proper (i.e., orientation preserving) rotations of $\mathbb{R}^{n}$. It is a compact group and carries a unique rotation invariant probability measure, which we denote by $\nu$. Let $G_{n}$ be the group of rigid motions of $\mathbb{R}^{n}$ and $\mu$ its invariant (or Haar) measure, normalized so that $\mu\left(\left\{g \in G_{n}: g x \in B^{n}\right\}\right)=\kappa_{n}$ for $x \in \mathbb{R}^{n}$. More explicitly, the mapping $\gamma: \mathbb{R}^{n} \times S O_{n} \rightarrow G_{n}$ defined by $\gamma(x, \vartheta) y:=\vartheta y+x$ for $y \in \mathbb{R}^{n}$ is a homeomorphism, and $\mu$ is the image measure of the product measure $\lambda_{n} \otimes \nu$ under $\gamma$.

By $\mathcal{L}_{q}^{n}$ we denote the Grassmannian of $q$-dimensional linear subspaces of $\mathbb{R}^{n}$, for $q \in\{0, \ldots, n\}$, and by $\nu_{q}$ its rotation invariant probability measure. Similarly, $\mathcal{E}_{q}^{n}$ is the space of $q$-flats in $\mathbb{R}^{n}$, and $\mu_{q}$ is its motion invariant measure, normalized so that $\mu_{q}\left(\left\{E \in \mathcal{E}_{q}^{n}: E \cap B^{n} \neq \emptyset\right\}\right)=\kappa_{n-q}$. This, too, we make more explicit. We choose a fixed subspace $L \in \mathcal{L}_{q}^{n}$ and denote by $L^{\perp}$ its orthogonal complement. The mappings $\beta_{q}: S O_{n} \rightarrow \mathcal{L}_{q}^{n}, \vartheta \mapsto \vartheta L$, and $\gamma_{q}: L^{\perp} \times S O_{n} \rightarrow \mathcal{E}_{q}^{n},(x, \vartheta) \mapsto \vartheta(L+x)$, are continuous and surjective. Now $\nu_{q}$ is the image measure of the invariant measure $\nu$ under $\beta_{q}$, and $\mu_{q}$ is the image measure of the product measure $\lambda_{n-q}^{L^{\perp}} \otimes \nu$ under $\gamma_{q}$, where $\lambda_{n-q}^{L^{\perp}}$ denotes Lebesgue measure on $L^{\perp}$.

Once the invariant measures $\mu$ and $\mu_{k}$ are available, it is of interest to determine, for convex bodies $K, M \in \mathcal{K}$, the total measures $\mu\left(\left\{g \in G_{n}\right.\right.$ : $K \cap g M \neq \emptyset\})$ and $\mu_{k}\left(\left\{E \in \mathcal{E}_{k}^{n}: K \cap E \neq \emptyset\right\}\right)$. We write these as the integrals

$$
\int_{G_{n}} \chi(K \cap g M) \mu(\mathrm{d} g) \quad \text { and } \quad \int_{\mathcal{E}_{k}^{n}} \chi(K \cap E) \mu_{k}(\mathrm{~d} E) \text {, }
$$

recalling that $\chi(K)=1$ for a convex body $K$ and $\chi(\emptyset)=0$. Since $\chi=V_{0}$, a more general task is to determine the integrals 


$$
\int_{G_{n}} V_{j}(K \cap g M) \mu(\mathrm{d} g) \quad \text { and } \quad \int_{\mathcal{E}_{k}^{n}} V_{j}(K \cap E) \mu_{k}(\mathrm{~d} E),
$$

for $j=0, \ldots, n$. For that, we use Hadwiger's characterization theorem. We begin with the latter integral. By

$$
\psi(K):=\int_{\mathcal{E}_{k}^{n}} V_{j}(K \cap E) \mu_{k}(\mathrm{~d} E) \quad \text { for } K \in \mathcal{K}
$$

we define a functional $\psi: \mathcal{K} \rightarrow \mathbb{R}$. It is not difficult to show that this functional is additive, motion invariant and continuous (for the continuity, compare the argument used in the proof of Theorem 1.4). Hadwiger's characterization theorem yields a representation

$$
\psi(K)=\sum_{r=0}^{n} c_{r} V_{r}(K)
$$

Here only one coefficient is different from zero. In fact, from

$$
\psi(K)=\int_{\mathcal{L}_{k}^{n}} \int_{L^{\perp}} V_{j}(K \cap(L+y)) \lambda_{n-k}(\mathrm{~d} y) \nu_{k}(\mathrm{~d} L)
$$

one sees that $\psi$ has the homogeneity property

$$
\psi(\alpha K)=\alpha^{n-k+j} \psi(K)
$$

for $\alpha>0$. Since $V_{k}$ is homogeneous of degree $k$, we deduce that $c_{r}=0$ for $r \neq n-k+j$. Thus, we have obtained

$$
\int_{\mathcal{E}_{k}^{n}} V_{j}(K \cap E) \mu_{k}(\mathrm{~d} E)=c V_{n-k+j}(K)
$$

with some constant $c$. In order to determine this constant, we choose for $K$ the unit ball $B^{n}$. For $\epsilon \geq 0$, the Steiner formula gives

$$
\sum_{j=0}^{n} \epsilon^{n-j} \kappa_{n-j} V_{j}\left(B^{n}\right)=V_{n}\left(B^{n}+\epsilon B^{n}\right)=(1+\epsilon)^{n} \kappa_{n}=\sum_{j=0}^{n} \epsilon^{n-j}\left(\begin{array}{l}
n \\
j
\end{array}\right) \kappa_{n},
$$

hence

$$
V_{j}\left(B^{n}\right)=\frac{\left(\begin{array}{c}
n \\
j
\end{array}\right) \kappa_{n}}{\kappa_{n-j}} \quad \text { for } j=0, \ldots, n
$$

Choosing $L \in \mathcal{L}_{k}^{n}$, we obtain

$$
c V_{n-k+j}\left(B^{n}\right)=\int_{\mathcal{E}_{k}^{n}} V_{j}\left(B^{n} \cap E\right) \mu_{k}(\mathrm{~d} E)
$$




$$
\begin{aligned}
& =\int_{S O_{n}} \int_{L^{\perp}} V_{j}\left(B^{n} \cap \vartheta(L+x)\right) \lambda_{n-k}(\mathrm{~d} x) \nu(\mathrm{d} \vartheta) \\
& =\int_{L^{\perp} \cap B^{n}}\left(1-\|x\|^{2}\right)^{j / 2} V_{j}\left(B^{n} \cap L\right) \lambda_{n-k}(\mathrm{~d} x) \\
& =\frac{\left(\begin{array}{c}
k \\
j
\end{array}\right) \kappa_{k}}{\kappa_{k-j}} \int_{L^{\perp} \cap B^{n}}\left(1-\|x\|^{2}\right)^{j / 2} \lambda_{n-k}(\mathrm{~d} x) .
\end{aligned}
$$

Introducing polar coordinates, we transform the latter integral into a Beta integral and finally obtain

$$
c=\frac{\left(\begin{array}{l}
k \\
j
\end{array}\right) \kappa_{k} \kappa_{n-k+j}}{V_{n-k+j}\left(B^{n}\right) \kappa_{k-j} \kappa_{j}}=c_{j, n}^{k, n-k+j},
$$

where we denote by

$$
c_{k, l}^{i, j}:=\frac{i ! \kappa_{i} j ! \kappa_{j}}{k ! \kappa_{k} l ! \kappa_{l}}
$$

a frequently occurring constant. By using the identity

$$
m ! \kappa_{m}=2^{m} \pi^{\frac{m-1}{2}} \Gamma\left(\frac{m+1}{2}\right),
$$

this can also be put in the form

$$
c_{k, l}^{i, j}=\frac{\Gamma\left(\frac{i+1}{2}\right) \Gamma\left(\frac{j+1}{2}\right)}{\Gamma\left(\frac{k+1}{2}\right) \Gamma\left(\frac{l+1}{2}\right)} .
$$

More generally, we define

$$
c_{s_{1}, \ldots, s_{k}}^{r_{1}, \ldots, r_{k}}:=\prod_{i=1}^{k} \frac{r_{i} ! \kappa_{r_{i}}}{s_{i} ! \kappa_{s_{i}}}
$$

This notation is only defined with the same number of upper and lower indices; hence, when $c_{s, n, \ldots, n}^{r_{1}, \ldots, r_{k}}$ appears, it is clear that the index $n$ is repeated $k-1$ times.

We have obtained the following result.

Theorem 1.3. Let $K \in \mathcal{K}$ be a convex body. For $k \in\{1, \ldots, n-1\}$ and $j \leq k$ the Crofton formula

$$
\int_{\mathcal{E}_{k}^{n}} V_{j}(K \cap E) \mu_{k}(\mathrm{~d} E)=c_{j, n}^{k, n-k+j} V_{n-k+j}(K)
$$

holds. 
The special case $j=0$ of (15) gives

$$
V_{m}(K)=c_{m, n-m}^{0, n} \int_{\mathcal{E}_{n-m}^{n}} \chi(K \cap E) \mu_{n-m}(\mathrm{~d} E)
$$

and thus provides an integral geometric interpretation of the intrinsic volumes: $V_{m}(K)$ is, up to a normalizing factor, the invariant measure of the set of $(n-m)$-flats intersecting $K$.

Using the explicit representation of the measure $\mu_{n-m}$, we can transform the representation (16) into

$$
V_{m}(K)=c_{m, n-m}^{0, n} \int_{\mathcal{L}_{m}^{n}} \lambda_{m}(K \mid L) \nu_{m}(\mathrm{~d} L)
$$

where $K \mid L$ denotes the image of $K$ under orthogonal projection to the subspace $L$. The special case $m=1$ shows that $V_{1}$, up to a factor, is the mean width.

From Hadwiger's characterization theorem, we now deduce a general kinematic formula, involving a functional on convex bodies that need not have any invariance property.

Theorem 1.4 (Hadwiger's general integral geometric theorem). If $\varphi$ : $\mathcal{K} \rightarrow \mathbb{R}$ is an additive continuous function, then

$$
\int_{G_{n}} \varphi(K \cap g M) \mu(\mathrm{d} g)=\sum_{k=0}^{n} \varphi_{n-k}(K) V_{k}(M)
$$

for $K, M \in \mathcal{K}$, where the coefficients $\varphi_{n-k}(K)$ are given by

$$
\varphi_{n-k}(K)=\int_{\mathcal{E}_{k}^{n}} \varphi(K \cap E) \mu_{k}(\mathrm{~d} E) .
$$

Proof. The $\mu$-integrability of the integrand in (18) can be shown without difficulty. Now we fix a convex body $K \in \mathcal{K}$ and define

$$
\psi(M):=\int_{G_{n}} \varphi(K \cap g M) \mu(\mathrm{d} g) \quad \text { for } M \in \mathcal{K} .
$$

Then $\psi: \mathcal{K} \rightarrow \mathbb{R}$ is obviously additive and motion invariant. To show the continuity of $\psi$, let $M \in \mathcal{K}$ be given. Let $G_{n}(K, M)$ be the set of all motions $g \in G_{n}$ for which $K \cap g M \neq \emptyset$ and $K$ and $g M$ can be separated weakly by a hyperplane. Let $\left(M_{j}\right)_{j \in \mathbb{N}}$ be a sequence in $\mathcal{K}$ converging to $M$, and let $g \in G_{n} \backslash G_{n}(K, M)$. Then $g M_{j} \rightarrow g M$ and hence $K \cap g M_{j} \rightarrow K \cap g M$ (see [51, Hilfssatz 2.1.3]), thus $\varphi\left(K \cap g M_{j}\right) \rightarrow \varphi(K \cap g M)$, for $j \rightarrow \infty$. 
Since $\mu\left(G_{n}(K, M)\right)=0$ ([51, Hilfssatz 2.1.4]), it follows from the bounded convergence theorem that $\psi\left(M_{j}\right) \rightarrow \psi(M)$. Thus, $\psi$ is continuous.

Now Theorem 1.2 yields the existence of constants $\varphi_{0}(K), \ldots, \varphi_{n}(K)$ so that

$$
\psi(M)=\sum_{i=0}^{n} \varphi_{n-i}(K) V_{i}(M)
$$

for all $M \in \mathcal{K}$. The constants depend, of course, on the given body $K$, and we now have to determine them.

Let $k \in\{0, \ldots, n\}$, and choose $L_{k} \in \mathcal{L}_{k}^{n}$. Let $B_{k}^{n}:=B^{n} \cap L_{k}$ and $M_{r}:=r B_{k}^{n}$ for $r>0$. Then

$$
\psi\left(M_{r}\right)=\sum_{i=0}^{n} \varphi_{n-i}(K) V_{i}\left(M_{r}\right)=\sum_{i=0}^{k} \varphi_{n-i}(K) r^{i} V_{i}\left(B_{k}^{n}\right) .
$$

On the other hand,

$$
\begin{aligned}
\psi\left(M_{r}\right) & =\int_{G_{n}} \varphi\left(K \cap g r B_{k}^{n}\right) \mu(\mathrm{d} g) \\
& =\int_{S O_{n}} \int_{\mathbb{R}^{n}} \varphi\left(K \cap\left(\vartheta r B_{k}^{n}+x\right)\right) \lambda_{n}(\mathrm{~d} x) \nu(\mathrm{d} \vartheta) \\
& =\int_{S O_{n}} \int_{L_{k}^{\perp}} \int_{L_{k}} \varphi\left(K \cap\left(\vartheta r B_{k}^{n}+\vartheta x_{1}+\vartheta x_{2}\right)\right) \lambda_{k}\left(\mathrm{~d} x_{1}\right) \lambda_{n-k}\left(\mathrm{~d} x_{2}\right) \nu(\mathrm{d} \vartheta) \\
& =\int_{S O_{n}} \int_{L_{k}} \int_{L_{k}} \varphi\left(K \cap\left[\vartheta r\left(B_{k}^{n}+x_{1}\right)+\vartheta x_{2}\right]\right) r^{k} \lambda_{k}\left(\mathrm{~d} x_{1}\right) \lambda_{n-k}\left(\mathrm{~d} x_{2}\right) \nu(\mathrm{d} \vartheta)
\end{aligned}
$$

Comparison gives

$$
\begin{aligned}
& \kappa_{k} \varphi_{n-k}(K) \\
& =\lim _{r \rightarrow \infty} \int_{S O_{n}} \int_{L_{k}^{\perp}} \int_{L_{k}} \varphi\left(K \cap\left[\vartheta r\left(B_{k}^{n}+x_{1}\right)+\vartheta x_{2}\right]\right) \lambda_{k}\left(\mathrm{~d} x_{1}\right) \lambda_{n-k}\left(\mathrm{~d} x_{2}\right) \nu(\mathrm{d} \vartheta) .
\end{aligned}
$$

For $r \rightarrow \infty$, we have

$$
\varphi\left(K \cap\left[\vartheta r\left(B_{k}^{n}+x_{1}\right)+\vartheta x_{2}\right]\right) \rightarrow\left\{\begin{array}{ll}
\varphi\left(K \cap \vartheta\left(L_{k}+x_{2}\right)\right) & \text { if } 0 \in \operatorname{relint} \vartheta\left(B_{k}^{n}+x_{1}\right) \\
0 & \text { if } 0 \notin \vartheta\left(B_{k}^{n}+x_{1}\right)
\end{array} .\right.
$$

Hence, the bounded convergence theorem gives

$$
\begin{aligned}
\kappa_{k} \varphi_{n-k}(K) & =\int_{S O_{n}} \int_{L_{k}^{\perp}} \varphi\left(K \cap \vartheta\left(L_{k}+x_{2}\right)\right) \lambda_{k}\left(\vartheta B_{k}^{n}\right) \lambda_{n-k}\left(\mathrm{~d} x_{2}\right) \nu(\mathrm{d} \vartheta) \\
& =\kappa_{k} \int_{\mathcal{E}_{k}^{n}} \varphi(K \cap E) \mu_{k}(\mathrm{~d} E),
\end{aligned}
$$


as asserted.

Hadwiger's general formula can be iterated, that is, extended to a finite number of moving convex bodies. For this, we need the following lemma.

Lemma 1.1. Let $r, s \in\{1, \ldots, n-1\}, r+s \geq n$, and let $f: \mathcal{E}_{r+s-n}^{n} \rightarrow \mathbb{R}$ be a nonnegative measurable function. Then

$$
\int_{\mathcal{E}_{r}^{n}} \int_{\mathcal{E}_{s}^{n}} f\left(E_{1} \cap E_{2}\right) \mu_{r}\left(\mathrm{~d} E_{1}\right) \mu_{s}\left(\mathrm{~d} E_{2}\right)=c_{r+s-n, n}^{r, s} \int_{\mathcal{E}_{r+s-n}^{n}} f(E) \mu_{r+s-n}(\mathrm{~d} E) .
$$

Proof. It follows from the uniqueness, up to a constant, of the invariant measure $\mu_{r+s-n}$ that such a result must be true with some constant, independent of $f$, in front of the second integral. The value of the constant is then determined by choosing $f(E):=V_{0}(K \cap E)$ for some $n$-dimensional convex body $K$ and applying formula (15) three times.

Theorem 1.5. Let $\varphi: \mathcal{K} \rightarrow \mathbb{R}$ be an additive continuous function, and let $K_{1}, \ldots, K_{k} \in \mathcal{K}, k \geq 2$, be convex bodies. Then

$$
\begin{aligned}
& \int_{G_{n}} \cdots \int_{G_{n}} \varphi\left(K_{1} \cap g_{2} K_{2} \cap \cdots \cap g_{k} K_{k}\right) \mu\left(\mathrm{d} g_{2}\right) \cdots \mu\left(\mathrm{d} g_{k}\right) \\
& =\sum_{\substack{r_{1}, \ldots, r_{k}=0 \\
r_{1}+\cdots+r_{k}=(k-1) n}}^{n} c_{n-r_{1}, n, \ldots, n}^{r_{2}, \ldots, r_{k}} \varphi_{r_{1}}\left(K_{1}\right) V_{r_{2}}\left(K_{2}\right) \cdots V_{r_{k}}\left(K_{k}\right),
\end{aligned}
$$

where the coefficients are given by (14).

Proof. The proof proceeds by induction with respect to $k$. Theorem 1.4 is the case $k=2$; the induction step uses Theorem 1.4 and Lemma 1.1 with $f(E)=\varphi\left(K_{1} \cap E\right)$, as well as Fubini's theorem.

In Theorems 1.4 and 1.5, we can choose for $\varphi$, in particular, the intrinsic volume $V_{j}$. In this case, the Crofton formula (15) tells us that

$$
\left(V_{j}\right)_{r}(K)=\int_{\mathcal{E}_{n-r}^{n}} V_{j}(K \cap E) \mu_{n-r}(\mathrm{~d} E)=c_{j, n}^{n-r, r+j} V_{r+j}(K) .
$$

Hence, we obtain the following results, which for greater clarity we formulate separately.

Theorem 1.6. Let $K, M \in \mathcal{K}$ be convex bodies, and let $j \in\{0, \ldots, n\}$. Then the principal kinematic formula

$$
\int_{G_{n}} V_{j}(K \cap g M) \mu(\mathrm{d} g)=\sum_{k=j}^{n} c_{j, n}^{k, n-k+j} V_{k}(K) V_{n-k+j}(M)
$$

holds. 
We note that the special case $j=0$, or

$$
\int_{G_{n}} \chi(K \cap g M) \mu(\mathrm{d} g)=\sum_{k=0}^{n} c_{0, n}^{k, n-k} V_{k}(K) V_{n-k}(M),
$$

gives the formula (3) stated in the introduction.

Theorem 1.7 (Iterated Kinematic Formula). Let $K_{1}, \ldots, K_{k} \in \mathcal{K}, k \geq$ 2 , be convex bodies, and let $j \in\{0, \ldots, n\}$. Then

$$
\begin{aligned}
& \int_{G_{n}} \cdots \int_{G_{n}} V_{j}\left(K_{1} \cap g_{2} K_{2} \cap \cdots \cap g_{k} K_{k}\right) \mu\left(\mathrm{d} g_{2}\right) \cdots \mu\left(\mathrm{d} g_{k}\right) \\
& =\sum_{\substack{m_{1}, \ldots, m_{k}=j \\
m_{1}+\cdots+m_{k}=(k-1) n+j}}^{n} c_{j, n, \ldots, n}^{m_{1}, \ldots, m_{k}} V_{m_{1}}\left(K_{1}\right) \cdots V_{m_{k}}\left(K_{k}\right) .
\end{aligned}
$$

Hints to the literature. The idea of deducing integral geometric formulae for convex bodies from a characterization of the intrinsic volumes essentially goes back to W. Blaschke. It was put on a solid basis by Hadwiger [12]. Hadwiger's general integral geometric theorem and its deduction from the characterization theorem appear in [15] and [16].

The standard source for integral geometry is [37]. An introduction to integral geometry in the spirit of these lectures, with a special view to applications in stochastic geometry, is given in [51].

\section{Localizations and extensions}

The envisaged applications of the kinematic formula (20) to stochastic geometry require its extension in several directions. In this section, we first treat a local version. It involves local versions of the intrinsic volumes, in the form of curvature measures and their generalizations. The rest of the section deals with extensions to non-convex sets.

\subsection{The kinematic formula for curvature measures}

The notion of the parallel body of a convex body $K$ can be generalized, by taking only those points $x$ into account for which the nearest point $p(K, x)$ in $K$ belongs to some specified set of points, and/or the normalized vector from $p(K, x)$ to $x$ belongs to some specified set of directions. Again there is a Steiner formula, and the coefficients define the curvature measures. In this section we show how the kinematic and Crofton formulae can be extended to these curvature measures. First we introduce a general version of curvature measures. 
By a support element of the convex body $K \in \mathcal{K}$ we understand a pair $(x, u)$ where $x$ is a boundary point of $K$ and $u$ is an outer unit normal vector of $K$ at $x$. The set of all support elements of $K$ is called the generalized normal bundle of $K$ and is denoted by Nor $K$. It is a subset of the product space $\Sigma:=\mathbb{R}^{n} \times S^{n-1}$.

Recall that $p(K, x)$ is the point in the convex body $K$ nearest to $x \in \mathbb{R}^{n}$ and that $d(K, x):=\|x-p(K, x)\|$ is the distance of $x$ from $K$. For $x \notin K$ we have $d(K, x)>0$, and we put $u(K, x):=(x-p(K, x)) / d(K, x)$; then $(p(K, x), u(K, x)) \in$ Nor $K$. For $\rho>0$ and each Borel set $S \subset \Sigma$, a local parallel set is now defined by

$$
M_{\rho}(K, S):=\left\{x \in K_{\rho} \backslash K:(p(K, x), u(K, x)) \in S\right\} .
$$

The Steiner formula extends as follows:

Theorem 2.1 (Local Steiner formula). For $K \in \mathcal{K}$, there are finite measures $\Xi_{0}(K, \cdot), \ldots, \Xi_{n-1}(K, \cdot)$ on $\Sigma$ such that, for $\rho \geq 0$ and every $S \in \mathcal{B}(\Sigma)$,

$$
\lambda_{n}\left(M_{\rho}(K, S)\right)=\sum_{m=0}^{n-1} \rho^{n-m} \kappa_{n-m} \Xi_{m}(K, S) .
$$

The measures $\Xi_{0}(K, \cdot), \ldots, \Xi_{n-1}(K, \cdot)$ are called the support measures or generalized curvature measures of $K$.

The principle of the proof is clear from the proof of Theorem 1.1, the Steiner formula; only the details are slightly more technical. We sketch here the main ideas. We write $\mu_{\rho}(K, S):=\lambda_{n}\left(M_{\rho}(K, S)\right)$ for $S \in \mathcal{B}(\Sigma)$. First, for a polytope $P$ one obtains

$$
\mu_{\rho}(K, S)=\sum_{m=0}^{n-1} \rho^{n-m} \kappa_{n-m} \Xi_{m}(P, S)
$$

if one puts

$\Xi_{m}(P, S):=\sum_{F \in \mathcal{F}_{m}(P)} \int_{F} \frac{\sigma_{n-1-m}\left(N(P, F) \cap\left\{u \in S^{n-1}:(y, u) \in S\right\}\right)}{(n-m) \kappa_{n-m}} \lambda_{m}(\mathrm{~d} y)$.

Next, one shows that $\mu_{\rho}(K, \cdot)$ is a measure on $\Sigma$ and that $\lim _{j \rightarrow \infty} K_{j}=K$ for convex bodies $K_{j}, K$ implies that the sequence $\left(\mu_{\rho}\left(K_{j}, \cdot\right)\right)_{j \in \mathbb{N}}$ converges weakly to $\mu_{\rho}(K, \cdot)$. Thus, the map $K \mapsto \mu_{\rho}(K, \cdot)$ is weakly continuous. Moreover, this map is additive. For each fixed $S \in \mathcal{B}(\Sigma)$, the function $\mu_{\rho}(\cdot, S)$ is measurable. One can now follow the arguments of Section 1.2 and establish the existence of the measures $\Xi_{0}(K, \cdot), \ldots, \Xi_{n-1}(K, \cdot)$ for general $K \in \mathcal{K}$ so that (22) holds. Proceeding essentially as for the intrinsic volumes (which correspond to the case $S=\Sigma$ ), one then shows that the maps $\Xi_{m}: \mathcal{K} \times \mathcal{B}(\Sigma) \rightarrow \mathbb{R}$ have the following properties: 
- Motion covariance: $\Xi_{m}(g K, g . S)=\Xi_{m}(K, S)$ for $g \in G_{n}$, where $g . S:=$ $\left\{\left(g x, g_{0} u\right):(x, u) \in S\right\}, g_{0}$ denoting the rotation part of $g$,

- Homogeneity: $\Xi_{m}(\alpha K, \alpha \cdot S)=\Xi_{m}(K, S)$ for $\alpha \geq 0$, where $\alpha \cdot S:=$ $\{(\alpha x, u):(x, u) \in S\}$,

- Weak continuity: $K_{j} \rightarrow K$ implies $\Xi_{m}\left(K_{j}, \cdot\right) \rightarrow \Xi_{m}(K, \cdot)$ weakly,

- $\Xi_{m}(\cdot, S)$ is additive, for each fixed $S \in \mathcal{B}(\Sigma)$,

- $\Xi_{m}(\cdot, S)$ is measurable, for each fixed $S \in \mathcal{B}(\Sigma)$.

In the following, we will mainly use the first of two natural specializations of the support measures, which are defined by

$$
\begin{aligned}
& \Phi_{m}(K, A):=\Xi_{m}\left(K, A \times S^{n-1}\right) \quad \text { for } A \in \mathcal{B}\left(\mathbb{R}^{n}\right), \\
& \Psi_{m}(K, B):=\Xi_{m}\left(K, \mathbb{R}^{n} \times B\right) \quad \text { for } B \in \mathcal{B}\left(S^{n-1}\right) .
\end{aligned}
$$

Thus, $\Phi_{m}(K, \cdot)$ is the image measure of $\Xi_{m}(K, \cdot)$ under the projection $(x, u) \mapsto x$, and $\Psi_{m}(K, \cdot)$ is the image measure of $\Xi_{m}(K, \cdot)$ under the projection $(x, u) \mapsto u$. The measure $\Phi_{m}(K, \cdot)$ is called the $m$ th curvature measure of $K$, and $\Psi_{m}(K, \cdot)$ is called the $m$ th area measure of $K$, but the reader should be warned that often the same terminology is used for differently normalized measures. In particular, the measure $S_{n-1}(K, \cdot)=2 \Psi(K, \cdot)$ is commonly known as the area measure of $K$.

The defining Steiner formula for the curvature measures can be written in the form

$$
\lambda_{n}\left(\left\{x \in K_{\rho}: p(K, x) \in A\right\}\right)=\sum_{m=0}^{n} \rho^{n-m} \kappa_{n-m} \Phi_{m}(K, A)
$$

for $A \in \mathcal{B}\left(\mathbb{R}^{n}\right)$. Here we have admitted all $x \in K_{\rho}$ with $p(K, x) \in A$ on the left side; therefore, the right side contains the term

$$
\Phi_{n}(K, A):=\lambda_{n}(K \cap A) .
$$

The reason for the name 'curvature measure' becomes clear if one considers a convex body $K$ whose boundary $\partial K$ is a regular hypersurface of class $C^{2}$ with positive positive Gauss-Kronecker curvature. In that case, the local parallel volume can be computed by differential-geometric means, and one obtains for $m=0, \ldots, n-1$ the representation

$$
\Phi_{m}(K, A)=\frac{\left(\begin{array}{c}
n \\
m
\end{array}\right)}{n \kappa_{n-m}} \int_{A \cap \partial K} H_{n-1-m} \mathrm{~d} S .
$$

Here, $H_{k}$ denotes the $k$ th normalized elementary symmetric function of the principal curvatures of $\partial K$, and $\mathrm{d} S$ is the volume form on $\partial K$. Thus the curvature measures are (up to normalizing factors) indefinite integrals of curvature functions, and they replace the latter in the non-smooth case. The corresponding representation for the area measures is 


$$
\Psi_{m}(K, B)=\frac{\left(\begin{array}{c}
n \\
m
\end{array}\right)}{n \kappa_{n-m}} \int_{B} s_{m} \mathrm{~d} \sigma_{n-1}
$$

for $B \in \mathcal{B}\left(S^{n-1}\right)$. Here, $s_{m}$ is the $m$ th normalized elementary symmetric function of the principal radii of curvature of $\partial K$, as a function of the outer unit normal vector.

For a polytope $P$, the explicit representation given above for the support measure $\Xi_{m}(P, \cdot)$ specializes to

$$
\Phi_{m}(P, A)=\sum_{F \in \mathcal{F}_{m}(P)} \gamma(F, P) \lambda_{m}(F \cap A)
$$

for $A \in \mathcal{B}\left(\mathbb{R}^{n}\right)$ and

$$
\Psi_{m}(P, B)=\sum_{F \in \mathcal{F}_{m}(P)} \frac{\sigma_{n-1-m}(N(P, F) \cap B) \lambda_{m}(F)}{(n-m) \kappa_{n-m}}
$$

for $B \in \mathcal{B}\left(S^{n-1}\right)$.

For arbitrary $K \in \mathcal{K}$, it is clear from (23) that the curvature measures $\Phi_{0}(K, \cdot), \ldots, \Phi_{n-1}(K, \cdot)$ are concentrated on the boundary of $K$. We mention without proof that the measures $\Phi_{0}(K, \cdot)$ and $\Phi_{n-1}(K, \cdot)$ have simple intuitive interpretations. Let $\mathcal{H}^{n-1}$ denote $(n-1)$-dimensional Hausdorff measure. If $\operatorname{dim} K \neq n-1$, then

$$
\Phi_{n-1}(K, A)=\frac{1}{2} \mathcal{H}^{n-1}(A \cap \partial K) .
$$

For $\operatorname{dim} K=n-1$, one trivially has $\Phi_{n-1}(K, A)=\mathcal{H}^{n-1}(A \cap \partial K)$. The measure $\Phi_{0}$ is the normalized area of the spherical image. Let $\sigma(K, A) \subset S^{n-1}$ denote the set of all outer unit normal vectors of $K$ at points of $A \cap \partial K$, then

$$
\Phi_{0}(K, A)=\frac{1}{n \kappa_{n}} \mathcal{H}^{n-1}(\sigma(K, A)) .
$$

Now we state the local version of Theorem 1.6.

Theorem 2.2. Let $K, M \in \mathcal{K}$ be convex bodies, let $j \in\{0, \ldots, n\}$, and let $A, B \in \mathcal{B}\left(\mathbb{R}^{n}\right)$ be Borel sets. Then the local principal kinematic formula

$$
\int_{G_{n}} \Phi_{j}(K \cap g M, A \cap g B) \mu(\mathrm{d} g)=\sum_{k=j}^{n} c_{j, n}^{k, n-k+j} \Phi_{k}(K, A) \Phi_{n-k+j}(M, B)
$$

holds. For $k \in\{1, \ldots, n-1\}$ and $j \leq k$ the local Crofton formula

$$
\int_{\mathcal{E}_{k}^{n}} \Phi_{j}(K \cap E, A \cap E) \mu_{k}(\mathrm{~d} E)=c_{j, n}^{k, n-k+j} \Phi_{n-k+j}(K, A)
$$

holds. The coefficients $c_{j, n}^{k, n-k+j}$ are those given by (12). 
We will describe the main ideas of a proof for the case of polytopes. The result for general convex bodies is then obtained by approximation, using the weak continuity of the curvature measures. We omit the details of this approximation, as well as all arguments concerning measurability, null sets, and integration techniques. Due to the explicit representation of the Haar measure $\mu$, the integral over $G_{n}$ in (28) can be split in the form

$$
\int_{G_{n}} f(g M, g B) \mu(\mathrm{d} g)=\int_{S O_{n}} \int_{\mathbb{R}^{n}} f(\vartheta M+x, \vartheta B+x) \lambda_{n}(\mathrm{~d} x) \nu(\mathrm{d} \vartheta) .
$$

First we treat only the inner integral. This gives us the opportunity to introduce some notions and results of translative integral geometry, which will be elaborated upon in Section 3.

Let $K, M \in \mathcal{K}$ be $n$-dimensional polytopes, and let $A, B \in \mathcal{B}\left(\mathbb{R}^{n}\right)$ be Borel sets. We have to investigate the integral

$$
I:=\int_{\mathbb{R}^{n}} \Phi_{j}(K \cap(M+x), A \cap(B+x)) \lambda_{n}(\mathrm{~d} x) .
$$

By (26), the $j$ th curvature measure of a polytope $P$ is given by

$$
\Phi_{j}(P, \cdot)=\sum_{F \in \mathcal{F}_{j}(P)} \gamma(F, P) \lambda_{F},
$$

where we have introduced the abbreviation

$$
\lambda_{F}(\cdot):=\lambda_{\operatorname{dim} F}(F \cap \cdot) .
$$

It follows that

$$
I=\int_{\mathbb{R}^{n}} \sum_{F^{\prime} \in \mathcal{F}_{j}(K \cap(M+x))} \gamma\left(F^{\prime}, K \cap(M+x)\right) \lambda_{F^{\prime}}(A \cap(B+x)) \lambda_{n}(\mathrm{~d} x) .
$$

The faces $F^{\prime} \in \mathcal{F}_{j}(K \cap(M+x))$ are precisely the $j$-dimensional sets of the form $F^{\prime}=F \cap(G+x)$ with a face $F \in \mathcal{F}_{k}(K)$ and a face $G \in \mathcal{F}_{i}(M)$, where $k, i \in\{j, \ldots, n\}$. We may assume that $k+i=n+j$, since only such pairs $F, G$ contribute to the integral. Therefore, we obtain

$$
\begin{aligned}
I= & \sum_{k=j}^{n} \sum_{F \in \mathcal{F}_{k}(K)} \sum_{G \in \mathcal{F}_{n-k+j}(M)} \\
& \int_{\mathbb{R}^{n}} \gamma(F \cap(G+x), K \cap(M+x)) \lambda_{F \cap(G+x)}(A \cap(B+x)) \lambda_{n}(\mathrm{~d} x) .
\end{aligned}
$$

In the integrand, we may assume that relint $F \cap \operatorname{relint}(G+x) \neq \emptyset$, since other vectors $x$ do not contribute to the integral, and in this case the common exterior angle 


$$
\gamma(F, G ; K, M):=\gamma(F \cap(G+x), K \cap(M+x))
$$

does not depend on $x$. Putting

$$
J(F, G):=\int_{\mathbb{R}^{n}} \lambda_{F \cap(G+x)}(A \cap(B+x)) \lambda_{n}(\mathrm{~d} x),
$$

we thus have

$$
I=\sum_{k=j}^{n} \sum_{F \in \mathcal{F}_{k}(K)} \sum_{G \in \mathcal{F}_{n-k+j}(M)} \gamma(F, G ; K, M) J(F, G) .
$$

To compute the integral $J(F, G)$ for given faces $F \in \mathcal{F}_{k}(K)$ and $G \in$ $\mathcal{F}_{n-k+j}(M)$, we decompose the space $\mathbb{R}^{n}$ in a way adapted to these faces and apply Fubini's theorem. The result is

$$
J(F, G)=[F, G] \lambda_{F}(A) \lambda_{G}(B),
$$

where the 'generalized sine function' $[F, G]$ is defined as follows.

Let $L, L^{\prime} \subset \mathbb{R}^{n}$ be two linear subspaces. We choose an orthonormal basis of $L \cap L^{\prime}$ and extend it to an orthonormal basis of $L$ and also to an orthonormal basis of $L^{\prime}$. Let $P$ denote the parallelepiped that is spanned by the vectors obtained in this way. We define $\left[L, L^{\prime}\right]:=\lambda_{n}(P)$. Then $\left[L, L^{\prime}\right]$ depends only on the subspaces $L$ and $L^{\prime}$. If $L+L^{\prime} \neq \mathbb{R}^{n}$, then $\left[L, L^{\prime}\right]=0$. We extend this definition to faces $F, G$ of polytopes by putting $[F, G]:=\left[L, L^{\prime}\right]$, where $L$ and $L^{\prime}$ are the linear subspaces which are translates of the affine hulls of $F$ and $G$, respectively.

Inserting the expression for $J(F, G)$ in the integral $I$, we end up with the following principal translative formula for polytopes.

Theorem 2.3. If $K, M \in \mathcal{K}$ are polytopes and $A, B \in \mathcal{B}\left(\mathbb{R}^{n}\right)$, then for $j \in$ $\{0, \ldots, n\}$,

$$
\begin{aligned}
\int_{\mathbb{R}^{n}} & \Phi_{j}(K \cap(M+x), A \cap(B+x)) \lambda_{n}(\mathrm{~d} x) \\
= & \sum_{k=j}^{n} \sum_{F \in \mathcal{F}_{k}(K)} \sum_{G \in \mathcal{F}_{n-k+j}(M)} \gamma(F, G ; K, M)[F, G] \lambda_{F}(A) \lambda_{G}(B) .
\end{aligned}
$$

The kinematic formula at which we are aiming requires, for polytopes, the computation of

$$
\begin{aligned}
& \int_{G_{n}} \Phi_{j}(K \cap g M, A \cap g B) \mu(\mathrm{d} g) \\
& \quad=\int_{S O_{n}} \int_{\mathbb{R}^{n}} \Phi_{j}(K \cap(\vartheta M+x), A \cap(\vartheta B+x)) \lambda_{n}(\mathrm{~d} x) \nu(\mathrm{d} \vartheta)
\end{aligned}
$$




$$
\begin{aligned}
= & \sum_{k=j}^{n} \sum_{F \in \mathcal{F}_{k}(K)} \sum_{G \in \mathcal{F}_{n-k+j}(M)} \lambda_{F}(A) \lambda_{G}(B) \\
& \int_{S O_{n}} \gamma(F, \vartheta G ; K, \vartheta M)[F, \vartheta G] \nu(\mathrm{d} \vartheta) .
\end{aligned}
$$

Here we have used the fact that $\lambda_{\vartheta G}(\vartheta B)=\lambda_{G}(B)$. The summands with $k=j$ or $k=n$ are easily determined; we get $\Phi_{j}(K, A) \Phi_{n}(M, B)$ for $k=j$ and $\Phi_{n}(K, A) \Phi_{j}(M, B)$ for $k=n$. The remaining integrals over the rotation group are determined in the following theorem.

Theorem 2.4. Let $K, M \in \mathcal{K}$ be polytopes, let $j \in\{0, \ldots, n-2\}, k \in\{j+$ $1, \ldots, n-1\}, F \in \mathcal{F}_{k}(K)$ and $G \in \mathcal{F}_{n-k+j}(M)$. Then

$$
\int_{S O_{n}} \gamma(F, \vartheta G ; K, \vartheta M)[F, \vartheta G] \nu(\mathrm{d} \vartheta)=c_{j, n}^{k, n-k+j} \gamma(F, K) \gamma(G, M),
$$

where $c_{j, n}^{k, n-k+j}$ is as in (12).

For this formula, a proof can be given which uses the fact that the spherical Lebesgue measure is, up to a constant factor, the only rotation invariant finite Borel measure on the sphere. In this way, one obtains (28), but with unknown coefficients instead of $c_{j, n}^{k, n-k+j}$. The values of these coefficients then follow from the fact that in the case $A=B=\mathbb{R}^{n}$ the result must coincide with (20). In this way, (28) is obtained. The local Crofton formula (29) can be deduced from (28) by an argument similar to that used in the proof of Theorem 1.4.

There is also a version of the local kinematic and Crofton formulae for support measures. Such a variant, which we mention only briefly, is possible if the intersection of Borel sets in $\mathbb{R}^{n}$ is replaced by a suitable law of composition for subsets of $\Sigma$, which is adapted to intersections of convex bodies. For $S, S^{\prime} \subset \Sigma$, let

$$
\begin{aligned}
S \wedge S^{\prime}:= & \left\{(x, u) \in \Sigma: \text { there are } u_{1}, u_{2} \in S^{n-1}\right. \text { with } \\
& \left.\left(x, u_{1}\right) \in S,\left(x, u_{2}\right) \in S^{\prime}, u \in \operatorname{pos}\left\{u_{1}, u_{2}\right\}\right\},
\end{aligned}
$$

where $\operatorname{pos}\left\{u_{1}, u_{2}\right\}:=\left\{\lambda_{1} u_{1}+\lambda_{2} u_{2}: \lambda_{1}, \lambda_{2} \geq 0\right\}$ is the positive hull of $\left\{u_{1}, u_{2}\right\}$. For a $q$-flat $E \in \mathcal{E}_{q}^{n}, q \in\{1, \ldots, n-1\}$, one defines

$$
\begin{aligned}
& S \wedge E:=\left\{(x, u) \in \Sigma: \text { there are } u_{1}, u_{2} \in S^{n-1}\right. \text { with } \\
&\left.\left(x, u_{1}\right) \in S, x \in E, u_{2} \in E^{\perp}, u \in \operatorname{pos}\left\{u_{1}, u_{2}\right\}\right\},
\end{aligned}
$$

where $E^{\perp}$ is the linear subspace totally orthogonal to $E$. Now for given convex bodies $K, K^{\prime} \in \mathcal{K}$, Borel sets $S \subset$ Nor $K$ and $S^{\prime} \subset$ Nor $K^{\prime}$, and for $j \in$ $\{0, \ldots, n-2\}$, the formula 


$$
\int_{G_{n}} \Xi_{j}\left(K \cap g K^{\prime}, S \wedge g S^{\prime}\right) \mu(\mathrm{d} g)=\sum_{k=j+1}^{n-1} c_{j, n}^{k, n-k+j} \Xi_{k}(K, S) \Xi_{n-k+j}\left(K^{\prime}, S^{\prime}\right)
$$

holds (for $j=n-1$, both sides would give 0 ). The local Crofton formula has the following extension. Let $K \in \mathcal{K}$ be a convex body, $k \in\{1, \ldots, n-1\}$, $j \in\{0, \ldots, k-1\}$, and let $S \subset$ Nor $K$ be a Borel set. Then

$$
\int_{\mathcal{E}_{k}^{n}} \Xi_{j}(K \cap E, S \wedge E) \mu_{k}(\mathrm{~d} E)=c_{j, n}^{k, n-k+j} \Xi_{n-k+j}(K, S) .
$$

Hints to the literature. For a more thorough introduction to support and curvature measures we refer to [39]. Detailed proofs of the kinematic and Crofton formulae for curvature measures of convex bodies are found in [39] and [51]. Formulae (33) and (34) are due to Glasauer [6], under an additional assumption. This assumption was removed by Schneider [42]. An analogue of Hadwiger's general integral geometric theorem for measure valued valuations on convex bodies was proved in [40]. A simpler proof was given in [5], and a generalization in [7].

\subsection{Additive extension to polyconvex sets}

So far, our integral geometric investigations were confined to convex bodies. In view of applications, this is a too narrow class of sets. The additivity of the intrinsic volumes and curvature measures permits us to extend these and the pertinent integral geometric intersection formulae to finite unions of convex bodies. This additive extension will be achieved in the present section.

By $\mathcal{R}$ we denote the system of all finite unions of convex bodies in $\mathbb{R}^{n}$ (including the empty set). The system $\mathcal{R}$, which is closed under finite unions and intersections, is called the convex ring (a questionable translation of the German 'Konvexring'). The elements of $\mathcal{R}$ will be called polyconvex sets.

Let $\varphi$ be a function on $\mathcal{R}$ with values in some abelian group. The function $\varphi$ is called additive or a valuation if

$$
\varphi(K \cup L)+\varphi(K \cap L)=\varphi(K)+\varphi(L)
$$

for $K, L \in \mathcal{R}$ and $\varphi(\emptyset)=0$. Every such valuation satisfies the inclusionexclusion principle

$$
\varphi\left(K_{1} \cup \cdots \cup K_{m}\right)=\sum_{r=1}^{m}(-1)^{r-1} \sum_{i_{1}<\cdots<i_{r}} \varphi\left(K_{i_{1}} \cap \cdots \cap K_{i_{r}}\right)
$$

for $K_{1}, \ldots, K_{m} \in \mathcal{R}$, as follows by induction.

If $\varphi$ is a valuation on $\mathcal{K}$, one may ask whether it has an extension to a valuation on $\mathcal{R}$. If such an extension exists and is also denoted by $\varphi$, then 
its values on $\mathcal{R}$ are given by (35), thus the extension is unique. Conversely, however, one cannot just employ (35) for the definition of such an extension, since the representation of an element of $\mathcal{R}$ in the form $K_{1} \cup \cdots \cup K_{m}$ with $K_{i} \in \mathcal{K}$ is in general not unique. Hence, the existence of an additive extension, if there is one, must be proved in a different way.

A simple example of a valuation on $\mathcal{R}$ is given by the indicator function. For $K \in \mathcal{R}$, let

$$
\mathbf{1}_{K}(x):= \begin{cases}1 & \text { for } x \in K, \\ 0 & \text { for } x \in \mathbb{R}^{n} \backslash K .\end{cases}
$$

For $K, L \in \mathcal{R}$ we trivially have $\mathbf{1}_{K \cup L}(x)+\mathbf{1}_{K \cap L}(x)=\mathbf{1}_{K}(x)+\mathbf{1}_{L}(x)$ for $x \in \mathbb{R}^{n}$. Hence, the mapping $\varphi: \mathcal{R} \rightarrow V, K \mapsto \mathbf{1}_{K}$, is an additive function on $\mathcal{R}$ with values in the vector space $V$ of finite linear combinations of indicator functions of polyconvex sets. By (35), $V$ consists of all linear combinations of indicator functions of convex bodies.

We will now prove a general extension theorem for valuations on $\mathcal{K}$.

Theorem 2.5. Let $X$ be a topological vector space, and let $\varphi: \mathcal{K} \rightarrow X$ be a continuous valuation. Then $\varphi$ has an additive extension to the convex ring $\mathcal{R}$.

Proof. Let $\varphi: \mathcal{K} \rightarrow X$ be additive and continuous. An essential part of the proof is the following

Proposition 2.1. The equality

$$
\sum_{i=1}^{m} \alpha_{i} \mathbf{1}_{K_{i}}=0
$$

with $m \in \mathbb{N}, \alpha_{i} \in \mathbb{R}, K_{i} \in \mathcal{K}$ implies

$$
\sum_{i=1}^{m} \alpha_{i} \varphi\left(K_{i}\right)=0 .
$$

Assume this proposition were false. Then there is a smallest number $m \in \mathbb{N}$, necessarily $m \geq 2$, for which there exist numbers $\alpha_{1}, \ldots, \alpha_{m} \in \mathbb{R}$ and convex bodies $K_{1}, \ldots, K_{m} \in \mathcal{K}$ such that

$$
\sum_{i=1}^{m} \alpha_{i} \mathbf{1}_{K_{i}}=0
$$

but

$$
\sum_{i=1}^{m} \alpha_{i} \varphi\left(K_{i}\right)=: a \neq 0
$$


Let $H \subset \mathbb{R}^{n}$ be a hyperplane with $K_{1} \subset \operatorname{int} H^{+}$, where $H^{+}, H^{-}$are the two closed halfspaces bounded by $H$. By (36) we have

$$
\sum_{i=1}^{m} \alpha_{i} \mathbf{1}_{K_{i} \cap H^{-}}=0, \quad \sum_{i=1}^{m} \alpha_{i} \mathbf{1}_{K_{i} \cap H}=0 .
$$

Since $K_{1} \cap H^{-}=\emptyset$ and $K_{1} \cap H=\emptyset$, each of these two sums has at most $m-1$ non-zero summands. From the minimality of $m$ (and from $\varphi(\emptyset)=0$ ) we get

$$
\sum_{i=1}^{m} \alpha_{i} \varphi\left(K_{i} \cap H^{-}\right)=0, \quad \sum_{i=1}^{m} \alpha_{i} \varphi\left(K_{i} \cap H\right)=0 .
$$

The additivity of $\varphi$ on $\mathcal{K}$ yields

$$
\sum_{i=1}^{m} \alpha_{i} \varphi\left(K_{i} \cap H^{+}\right)=a,
$$

whereas (36) gives

$$
\sum_{i=1}^{m} \alpha_{i} \mathbf{1}_{K_{i} \cap H^{+}}=0 .
$$

A standard separation theorem for convex bodies implies the existence of a sequence $\left(H_{j}\right)_{j \in \mathbb{N}}$ of hyperplanes with $K_{1} \subset \operatorname{int} H_{j}^{+}$for $j \in \mathbb{N}$ and

$$
K_{1}=\bigcap_{j=1}^{\infty} H_{j}^{+} .
$$

If the argument that has led us from (36), (37) to (39), (38) is applied $k$-times, we obtain

$$
\sum_{i=1}^{m} \alpha_{i} \varphi\left(K_{i} \cap \bigcap_{j=1}^{k} H_{j}^{+}\right)=a .
$$

For $k \rightarrow \infty$ this yields

$$
\sum_{i=1}^{m} \alpha_{i} \varphi\left(K_{i} \cap K_{1}\right)=a
$$

since

$$
\lim _{k \rightarrow \infty} K_{i} \cap \bigcap_{j=1}^{k} H_{j}^{+}=K_{i} \cap K_{1}
$$

in the sense of the Hausdorff metric (if $K_{i} \cap K_{1} \neq \emptyset$, otherwise use $\varphi(\emptyset)=0$ ) and $\varphi$ is continuous. Equality (36) implies

$$
\sum_{i=1}^{m} \alpha_{i} \mathbf{1}_{K_{i} \cap K_{1}}=0 .
$$


The procedure leading from (36) and (37) to (41) and (40) can be repeated, replacing the bodies $K_{i}$ and $K_{1}$ by $K_{i} \cap K_{1}$ and $K_{2}$, then by $K_{i} \cap K_{1} \cap K_{2}$ and $K_{3}$, and so on. Finally one obtains

$$
\sum_{i=1}^{m} \alpha_{i} \mathbf{1}_{K_{1} \cap \cdots \cap K_{m}}=0
$$

and

$$
\sum_{i=1}^{m} \alpha_{i} \varphi\left(K_{1} \cap \cdots \cap K_{m}\right)=a
$$

(because of $\left.K_{i} \cap K_{1} \cap \cdots \cap K_{m}=K_{1} \cap \cdots \cap K_{m}\right)$. Now $a \neq 0$ implies $\sum_{i=1}^{m} \alpha_{i} \neq 0$ and hence $\mathbf{1}_{K_{1} \cap \cdots \cap K_{m}}=0$ by the first relation, but this yields $\varphi\left(K_{1} \cap \cdots \cap\right.$ $\left.K_{m}\right)=0$, contradicting the second relation. This completes the proof of the proposition.

Now we consider the real vector space $V$ of all finite linear combinations of indicator functions of elements of $\mathcal{K}$. For $K \in \mathcal{R}$ we have $\mathbf{1}_{K} \in V$, as noted earlier. For fixed $f \in V$ we choose a representation

$$
f=\sum_{i=1}^{m} \alpha_{i} \mathbf{1}_{K_{i}}
$$

with $m \in \mathbb{N}, \alpha_{i} \in \mathbb{R}, K_{i} \in \mathcal{K}^{n}$ and define

$$
\tilde{\varphi}(f):=\sum_{i=1}^{m} \alpha_{i} \varphi\left(K_{i}\right)
$$

The proposition proved above shows that this definition is possible, since the right-hand side does not depend on the special representation chosen for $f$. Evidently, $\tilde{\varphi}: V \rightarrow X$ is a linear map satisfying $\tilde{\varphi}\left(\mathbf{1}_{K}\right)=\varphi(K)$ for $K \in \mathcal{K}$. We can now extend $\varphi$ from $\mathcal{K}^{n}$ to $\mathcal{R}$ by defining

$$
\varphi(K):=\tilde{\varphi}\left(\mathbf{1}_{K}\right) \quad \text { for } K \in \mathcal{R} .
$$

By the linearity of $\tilde{\varphi}$ and the additivity of the map $K \mapsto \mathbf{1}_{K}$ we obtain, for $K, M \in \mathcal{R}$,

$$
\begin{aligned}
\varphi(K \cup M)+\varphi(K \cap M) & =\tilde{\varphi}\left(\mathbf{1}_{K \cup M}\right)+\tilde{\varphi}\left(\mathbf{1}_{K \cap M}\right)=\tilde{\varphi}\left(\mathbf{1}_{K \cup M}+\mathbf{1}_{K \cap M}\right) \\
& =\tilde{\varphi}\left(\mathbf{1}_{K}+\mathbf{1}_{M}\right)=\tilde{\varphi}\left(\mathbf{1}_{K}\right)+\tilde{\varphi}\left(\mathbf{1}_{M}\right) \\
& =\varphi(K)+\varphi(M) .
\end{aligned}
$$

Thus $\varphi$ is additive on $\mathcal{R}$.

The extension theorem can be applied to the map $K \mapsto \Phi_{m}(K, \cdot)$ from $\mathcal{K}$ into the vector space of finite signed measures on $\mathcal{B}\left(\mathbb{R}^{n}\right)$ with the weak topology, since this map is additive and continuous. Hence, the curvature measures 
have unique additive extensions, as finite signed measures, to the convex ring $\mathcal{R}$. The values of the extension can be obtained from the inclusion-exclusion principle (35), which we now write in a more concise form.

For $m \in \mathbb{N}$, let $S(m)$ denote the set of all non-empty subsets of $\{1, \ldots, m\}$. For $v \in S(m)$, let $|v|:=\operatorname{card} v$. If $K_{1}, \ldots, K_{m}$ are given, we write

$$
K_{v}:=K_{i_{1}} \cap \cdots \cap K_{i_{m}} \quad \text { for } v=\left\{i_{1}, \ldots, i_{r}\right\} \in S(m) .
$$

With these conventions, the inclusion-exclusion principle (35) for an additive function $\varphi$ can be written in the form

$$
\varphi\left(K_{1} \cup \cdots \cup K_{m}\right)=\sum_{v \in S(m)}(-1)^{|v|-1} \varphi\left(K_{v}\right) .
$$

We can now easily extend our integral geometric formulae for curvature measures (which includes the case of intrinsic volumes) to polyconvex sets. Let $K \in \mathcal{R}$. We choose a representation

$$
K=\bigcup_{i=1}^{m} K_{i}
$$

with convex bodies $K_{1}, \ldots, K_{m}$. Since $\Phi_{k}$ is additive on $\mathcal{R}$, the inclusionexclusion principle gives

$$
\Phi_{k}(K, \cdot)=\sum_{v \in S(m)}(-1)^{|v|-1} \Phi_{k}\left(K_{v}, \cdot\right) .
$$

Now let $M \in \mathcal{K}$ be a convex body, and let $A, B \in \mathcal{B}\left(\mathbb{R}^{n}\right)$. Since the principal kinematic formula holds for convex bodies, we obtain

$$
\begin{aligned}
& \int_{G_{n}} \Phi_{j}(K \cap g M, A \cap g B) \mu(\mathrm{d} g) \\
& =\int_{G_{n}} \Phi_{j}\left(\bigcup_{i=1}^{m}\left(K_{i} \cap g M\right), A \cap g B\right) \mu(\mathrm{d} g) \\
& =\int_{G_{n}} \sum_{v \in S(m)}(-1)^{|v|-1} \Phi_{j}\left(K_{v} \cap g M, A \cap g B\right) \mu(\mathrm{d} g) \\
& =\sum_{v \in S(m)}(-1)^{|v|-1} \sum_{k=j}^{n} c_{j, n}^{k, n-k+j} \Phi_{k}\left(K_{v}, A\right) \Phi_{n-k+j}(M, B) \\
& =\sum_{k=j}^{n} c_{j, n}^{k, n-k+j} \Phi_{k}(K, A) \Phi_{n-k+j}(M, B) .
\end{aligned}
$$

Hence, the kinematic formula holds for $K \in \mathcal{R}$ and $M \in \mathcal{K}$. In a similar way, it can now be extended to $K \in \mathcal{R}$ and $M \in \mathcal{R}$. An analogous extension is possible for the Crofton formulae. 
Hints to the literature. In adopting the name 'polyconvex' for the elements of the convex ring, we followed Klain and Rota [25], who in turn followed E. de Giorgi. The extension theorem 2.5 and its proof reproduced here are due to Groemer [10]. For the support measures, and thus for the curvature measures, a more explicit construction of an additive extension to polyconvex sets is found in Section 4.4 of [39]. It is based on an extension of the Steiner formula for polyconvex sets, with the Lebesgue measure replaced by the integral of the multiplicity function that arises from additive extension of the indicator function of a parallel set. An application of this extension in stochastic geometry appears in [38].

\subsection{Curvature Measures for More General Sets}

The class of sets for which the curvature measures $\Phi_{j}$ have been defined, and the local principal kinematic formula (28) has been proved is, up to now, the convex ring, which consists of the finite unions of convex bodies. These polyconvex sets may seem sufficiently general for the simpler purposes of applied stochastic geometry, since sets consisting of very many very small convex bodies can be considered as sufficiently good models for real materials. Nevertheless, from theoretical as well as practical viewpoints, it seems desirable to extend the definition of curvature measures and the validity of kinematic formulae beyond the domain of the convex ring. In this subsection we briefly describe such extensions.

Our approach to the curvature measures involved two steps: the definition via the local Steiner formula (23) (or (22), more generally) and additive extension. The local Steiner formula expresses the Lebesgue measure of a local parallel set, and the definition of the latter makes essential use of the nearest point map $p(K, \cdot)$ of a convex body $K$, and thus of the fact that to each point $x \in \mathbb{R}^{n}$ there is a unique nearest point in $K$. It can be proved that a closed set $A \subset \mathbb{R}^{n}$ with the property that to each point of $\mathbb{R}^{n}$ there is a unique nearest point in $A$, must necessarily be convex ([39], Theorem 1.2.4). At first glance, this seems to indicate that the Steiner formula approach is restricted to convex sets. However, this is not the case. In fact, it is sufficient to have the Steiner formula (23) for small positive values of the distance $\rho$. This leads us to the sets with positive reach.

Let $K \subset \mathbb{R}^{n}$ be a nonempty closed set. The reach of $K$, denoted by $\operatorname{reach}(K)$, is the largest number $\rho$ (or $\infty$ ) such that to each $x \in \mathbb{R}^{n}$ with distance $d(K, x)$ from $x$ to $K$ smaller than $\rho$, there is a unique point in $K$ nearest to $x$; this point is then denoted by $p(K, x)$. If $\operatorname{reach}(K)>0$, then $K$ is called a set with positive reach.

Let $K \subset \mathbb{R}^{n}$ be a compact set with positive reach. For every Borel set $A \subset \mathbb{R}^{n}$ and for $0 \leq \rho<\operatorname{reach}(K)$ one has a polynomial expansion

$$
\lambda_{n}\left(\left\{x \in \mathbb{R}^{n}: d(K, x) \leq \rho \text { and } p(K, x) \in A\right\}\right)=\sum_{m=0}^{n} \rho^{n-m} \kappa_{n-m} \Phi_{m}(K, A),
$$


and this defines the curvature measures $\Phi_{0}(K, \cdot), \ldots, \Phi_{n}(K, \cdot)$ of $K$. If $K$ is convex, these are the known positive measures; in the general case, they are finite signed measures on $\mathcal{B}\left(\mathbb{R}^{n}\right)$. Several of the properties of the curvature measures of convex bodies carry over to the curvature measures of compact sets with positive reach. We mention here only the extension of the principal kinematic formula: If $K, M \subset \mathbb{R}^{n}$ are compact sets with positive reach, then $K \cap g M$ is a set with positive reach for $\mu$-almost all rigid motions $g \in G_{n}$, and the kinematic formula (28) holds for Borel sets $A, B$.

A Steiner type formula for arbitrary closed sets, involving a generalized version of the support measures, is treated in [19]. Since it is applicable in stochastic geometry, we will describe it here, without proofs. To explain the principal ideas, we start with a convex body $K$ which has a regular boundary of class $C^{2}$ with positive Gauss-Kronecker curvature. First we state a more general version of the local Steiner formula. Instead of the volume of a local parallel set, we consider the integral $\int_{\mathbb{R}^{n} \backslash K} f \mathrm{~d} \lambda_{n}$ of a bounded measurable function $f$ with compact support. It is intuitively clear, and can be proved, that

$$
\int_{\mathbb{R}^{n} \backslash K} f \mathrm{~d} \lambda_{n}=\int_{0}^{\infty} \int_{\partial K_{t}} f \mathrm{~d} S_{t} \mathrm{~d} t,
$$

where $\mathrm{d} S_{t}$ denotes the volume form of the boundary of the parallel body $K_{t}$. We transform the inner integral into an integral over the sphere $S^{n-1}$. For $u \in S^{n-1}$, let $x_{u}$ be the (unique) boundary point of $K$ with outer unit normal vector $u$. Then

$$
\int_{\partial K_{t}} f \mathrm{~d} S_{t}=\int_{S^{n-1}} f\left(x_{u}+t u\right) \prod_{i=1}^{n-1} r_{i}^{(t)}(u) \sigma_{n-1}(\mathrm{~d} u)
$$

where $r_{i}^{(t)}(u), i=1, \ldots, n-1$, are the principal radii of curvature of $\partial K_{t}$ at $x_{u}+t u$. They are given by $r_{i}^{(t)}(u)=r_{i}^{(0)}(u)+t$, hence

$$
\prod_{i=1}^{n-1} r_{i}^{(t)}(u)=\sum_{i=0}^{n-1}\left(\begin{array}{c}
n-1 \\
i
\end{array}\right) t^{n-i-1} s_{i}(u)
$$

with $s_{i}$ as in (25). The result

$$
\int_{\mathbb{R}^{n} \backslash K} f \mathrm{~d} \lambda_{n}=\sum_{i=0}^{n-1}\left(\begin{array}{c}
n-1 \\
i
\end{array}\right) \int_{0}^{\infty} \int_{S^{n-1}} t^{n-i-1} f\left(x_{u}+t u\right) s_{i}(u) \sigma_{n-1}(\mathrm{~d} u) \mathrm{d} t
$$

can be written as an integral over the generalized normal bundle Nor $K$ with respect to the support measures, using (25). Setting $\omega_{m}:=m \kappa_{m}$, we get

$$
\int_{\mathbb{R}^{n} \backslash K} f \mathrm{~d} \lambda_{n}=\sum_{i=0}^{n-1} \omega_{n-i} \int_{0}^{\infty} \int_{\text {Nor } K} t^{n-i-1} f(x+t u) \Xi_{i}(K, \mathrm{~d}(x, u)) \mathrm{d} t .
$$


There is nothing in this formula which refers to the smoothness assumptions made for $K$. In fact, (44) can be extended to general convex bodies. The special choice

$$
f(x):=\mathbf{1}_{S}(p(K, x), u(K, x)) \mathbf{1}_{K_{\rho} \backslash K}(x)
$$

then gives the local Steiner formula (22).

Heuristically, one would expect that (44) remains true for a non-convex closed set $A$, provided that the function $f$ has its support in a region where the nearest point in $A$ is uniquely determined. Fortunately, the points where the latter does not hold can be neglected. So we assume now only that $A \subset \mathbb{R}^{n}$ is a nonempty closed set. As for convex bodies, we define $d(A, x):=\min \{\|a-x\|$ : $a \in A\}$, and we let $p(A, x):=a$ whenever $a$ is a uniquely determined point in $A$ nearest to $x$. If $d(A, x)>0$ and $p(A, x)$ exists, we define $u(A, x):=$ $(x-p(A, x)) / d(A, x)$. The exoskeleton $\operatorname{exo}(A)$ of $A$ is defined as the set of all points $x$ of $\mathbb{R}^{n} \backslash A$ for which $p(A, x)$ does not exist (because there is more than one point in $A$ nearest to $x$ ). One can prove that $\operatorname{exo}(A)$ has $n$ dimensional Lebesgue measure zero. The generalized normal bundle of $A$ is defined by

$$
\text { Nor } A:=\{(p(A, x), u(A, x)): x \notin A \cup \operatorname{exo}(A)\},
$$

and the reach function $\delta(A, \cdot): \mathbb{R}^{n} \times S^{n-1} \rightarrow[0, \infty]$ of $A$ by

$$
\delta(A, x, u):=\inf \{t \geq 0: x+t u \in \operatorname{exo}(A)\} \quad \text { if }(x, u) \in \operatorname{Nor} A,
$$

(with $\inf \emptyset=\infty)$ and $\delta(A, x, u):=0$ if $(x, u) \notin \operatorname{Nor} A$.

In [19], the support measures $\Xi_{0}(A, \cdot), \ldots, \Xi_{n-1}(A, \cdot)$ of $A$ are introduced as real-valued, $\sigma$-additive set functions on the system of all Borel sets in $\Sigma$ which are contained in

$$
(\Sigma \backslash \operatorname{Nor} A) \cup\{(x, u): x \in B, \delta(A, x, u) \geq s\}
$$

for some $s>0$ and some compact $B \subset \mathbb{R}^{n}$. These signed measures vanish on every Borel subset of $\Sigma \backslash$ Nor $A$. Denoting by $\left|\Xi_{i}\right|$ the total variation measure of $\Xi_{i}$ and putting $a \wedge b:=\min \{a, b\}$ for $a, b \in \mathbb{R}$, we formulate the following result from [19], which is a far-reaching generalization of the Steiner formula.

Theorem 2.6. The support measures $\Xi_{0}(A, \cdot), \ldots, \Xi_{n-1}(A, \cdot)$ of a nonempty closed set $A \subset \mathbb{R}^{n}$ satisfy

$$
\int_{\text {Nor } A} \mathbf{1}_{B}(x)(\delta(A, x, u) \wedge r)^{n-j}\left|\Xi_{j}\right|(A, \mathrm{~d}(x, u))<\infty
$$

for all compact sets $B \subset \mathbb{R}^{n}$ and all $r>0(j=0, \ldots, n-1)$, and

$$
\begin{aligned}
\int_{\mathbb{R}^{n} \backslash A} f \mathrm{~d} \lambda_{n}= & \sum_{i=0}^{n-1} \omega_{n-i} \int_{0}^{\infty} \int_{\operatorname{Nor} A} t^{n-i-1} \mathbf{1}\{t<\delta(A, x, u)\} \\
& \times f(x+t u) \Xi_{i}(A, \mathrm{~d}(x, u)) \mathrm{d} t
\end{aligned}
$$

for every measurable bounded function $f: \mathbb{R}^{n} \rightarrow \mathbb{R}$ with compact support. 
The proof, for which we must refer to [19], makes essential use of the fact that for a given compact set $A$ there exists a sequence $\left(A_{k}\right)_{k \in \mathbb{N}}$ of sets of positive reach such that

$$
\text { Nor } A \subset \bigcup_{k \in \mathbb{N}} \operatorname{Nor} A_{k}
$$

and, for $(x, u) \in \operatorname{Nor} A$,

$$
\delta(A, x, u) \leq \sup \left\{\operatorname{reach}\left(A_{k}\right):(x, u) \in \operatorname{Nor} A_{k}, k \in \mathbb{N}\right\} .
$$

In particular, Nor $A$ is countably $(n-1)$-rectifiable, so that generalized curvatures, as in [60], can be defined.

Hints to the literature. Sets with positive reach and their curvature measures were introduced by Federer [3], who also obtained kinematic and Crofton formulae for these measures. His theory has been further developed in the work of Martina Zähle and co-authors. This work treats current representations of Federer's curvature measures in [60], a short proof of the principal kinematic formula for sets with positive reach in [36], and extensions to certain finite unions of sets with positive reach in [61], [34].

There have been a number of successful attempts to define curvature measures, and to obtain kinematic formulae, for very general and quite abstract classes of sets. We refer to the brief survey in [22] (Subsection 2.1) and the references given there.

\section{Translative Integral Geometry}

The simple and elegant form of the kinematic formulae (20) and (28), in particular the separation of the two involved convex bodies on the right-hand sides, is due to the fact that the integrals are with respect to the invariant measure on the motion group. The stochastic geometry of stationary but not necessarily isotropic random sets requires analogous investigations with respect to the translation group, for example, the determination of the integrals

$$
\int_{\mathbb{R}^{n}} V_{j}(K \cap(M+t)) \lambda_{n}(\mathrm{~d} t) .
$$

In order to achieve a more concise form of translative formulae, we use in the following an operator notation for translations, namely

$$
x M:=M+x \quad \text { for } M \subset \mathbb{R}^{n} \text { and } x \in \mathbb{R}^{n} .
$$

Recall that the indicator function of a set $A \subset \mathbb{R}^{n}$ is denoted by $\mathbf{1}_{A}$. We write $M^{*}:=\left\{y \in \mathbb{R}^{n}:-y \in M\right\}$ for the reflection of a set $M$ in 0 .

The cases $j=n$ and $j=n-1$ of the integral (45) are still simple. We have $\mathbf{1}_{t M}(x)=\mathbf{1}_{x M^{*}}(t)$ and hence, by Fubini's theorem, 


$$
\begin{aligned}
& \int_{\mathbb{R}^{n}} V_{n}(K \cap t M) \lambda_{n}(\mathrm{~d} t)=\int_{\mathbb{R}^{n}} \int_{\mathbb{R}^{n}} \mathbf{1}_{K \cap t M}(x) \lambda_{n}(\mathrm{~d} x) \lambda_{n}(\mathrm{~d} t) \\
& \quad=\int_{\mathbb{R}^{n}} \int_{\mathbb{R}^{n}} \mathbf{1}_{K}(x) \mathbf{1}_{x M^{*}}(t) \lambda_{n}(\mathrm{~d} t) \lambda_{n}(\mathrm{~d} x)=\int_{\mathbb{R}^{n}} \mathbf{1}_{K}(x) V_{n}\left(x M^{*}\right) \lambda_{n}(\mathrm{~d} x) \\
& =V_{n}\left(M^{*}\right) \int_{\mathbb{R}^{n}} \mathbf{1}_{K}(x) \lambda_{n}(\mathrm{~d} x),
\end{aligned}
$$

thus

$$
\int_{\mathbb{R}^{n}} V_{n}(K \cap t M) \lambda_{n}(\mathrm{~d} t)=V_{n}(K) V_{n}(M) .
$$

With only slightly more effort one can show that

$$
\int_{\mathbb{R}^{n}} V_{n-1}(K \cap t M) \lambda_{n}(\mathrm{~d} t)=V_{n-1}(K) V_{n}(M)+V_{n}(K) V_{n-1}(M) .
$$

However, for the functional $V_{0}=\chi$, a separation of $K$ and $M$ on the righthand side does not take place. Since $\chi(K \cap t M)=\mathbf{1}_{K+M^{*}}(t)$, we have

$$
\int_{\mathbb{R}^{n}} \chi(K \cap t M) \lambda_{n}(\mathrm{~d} t)=V_{n}\left(K+M^{*}\right) .
$$

Convex geometry tells us that

$$
V_{n}\left(K+M^{*}\right)=\sum_{i=0}^{n}\left(\begin{array}{c}
n \\
i
\end{array}\right) V_{i}\left(K, M^{*}\right)
$$

with

$$
V_{i}\left(K, M^{*}\right):=V(\underbrace{K, \ldots, K}_{i}, \underbrace{M^{*}, \ldots, M^{*}}_{n-i})
$$

where the function $V: \mathcal{K}^{n} \rightarrow \mathbb{R}$ is the so-called mixed volume. The essential observation for us is that the obtained expression cannot be simplified further. Thus, in translative integral geometry we must live with more complicated functionals, depending on several convex bodies simultaneously. A translative formula for curvature measures, which we will now study, necessarily involves new measures depending on several convex bodies.

\subsection{The Principal Translative Formula for Curvature Measures}

We have already obtained a translative formula for the curvature measures of polytopes, namely Theorem 2.3. We rewrite this result in a form that is convenient for the following. Let $K, M \in \mathcal{K}$ be polytopes, let $A, B \subset \mathbb{R}^{n}$ be Borel sets, and let $j \in\{0, \ldots, n\}$; then

$$
\int_{\mathbb{R}^{n}} \Phi_{j}(K \cap x M, A \cap x B) \lambda_{n}(\mathrm{~d} x)=\sum_{m=j}^{n} \Phi_{m, n+j-m}^{(j)}(K, M ; A \times B),
$$


where for $m, k \in\{j, \ldots, n\}$ with $m+k=n+j$ we have introduced measures $\Phi_{m, k}^{(j)}(K, M ; \cdot)$ on $\left(\mathbb{R}^{n}\right)^{2}$ by

$$
\Phi_{m, k}^{(j)}(K, M ; \cdot):=\sum_{F \in \mathcal{F}_{m}(K)} \sum_{G \in \mathcal{F}_{k}(M)} \gamma(F, G ; K, M)[F, G] \lambda_{F} \otimes \lambda_{G} .
$$

To extend this to more than two polytopes, we first extend the notation. For a polyhedral set $P$ (a nonempty intersection of finitely many closed halfspaces) in $\mathbb{R}^{n}$ and for a face $F$ of $P$, let $\gamma(F, P)$ be the (normalized) exterior angle of $P$ at $F$, defined by (5). If $K_{1}, \ldots, K_{k}$ are polyhedral sets and $F_{i}$ is a face of $K_{i}$ for $i=1, \ldots, k$, we choose points $x_{i} \in \operatorname{relint} F_{i}$ for $i=1, \ldots, k$ and define the common exterior angle $\gamma\left(F_{1}, \ldots, F_{k} ; K_{1}, \ldots, K_{k}\right)$ by

$$
\gamma\left(F_{1}, \ldots, F_{k} ; K_{1}, \ldots, K_{k}\right):=\gamma\left(\bigcap_{i=1}^{k}\left(F_{i}-x_{i}\right), \bigcap_{i=1}^{k}\left(K_{i}-x_{i}\right)\right) .
$$

This definition does not depend on the choice of the points $x_{i}$.

Further, we need the notion of the determinant of subspaces, extending the definition of the generalized sine function $[\cdot, \cdot]$ given in Subsection 2.1. Let $L_{1}, \ldots, L_{k}$ be linear subspaces of $\mathbb{R}^{n}$ with $\sum_{i=1}^{k} \operatorname{dim} L_{i}=: m \leq n$. Choose an orthonormal basis in each $L_{i}$ (the empty set if $\operatorname{dim} L_{i}=0$ ) and let $\operatorname{det}\left(L_{1}, \ldots, L_{k}\right)$ be the $m$-dimensional volume of the parallelepiped spanned by the union of these bases ( 1 , by definition, if $\operatorname{dim} L_{i}=0$ for $\left.i=1, \ldots, k\right)$. Then one defines

$$
\left[L_{1}, \ldots, L_{k}\right]:=\operatorname{det}\left(L_{1}^{\perp}, \ldots, L_{k}^{\perp}\right) \quad \text { if } \sum_{i=1}^{k} \operatorname{dim} L_{i} \geq(k-1) n
$$

and $\left[L_{1}, \ldots, L_{k}\right]:=0$ if $\sum_{i=1}^{k} \operatorname{dim} L_{i}<(k-1) n$. Obviously, any $n$-dimensional argument of $\left[L_{1}, \ldots, L_{k}\right]$ can be deleted without changing the value. We also note that $[L]=1$ and that $\left[L_{1}, \ldots, L_{k}\right]=0$ if $L_{1}, \ldots, L_{k}$ are not in general relative position (the subspaces $L_{1}, \ldots, L_{k}$ are in general relative position if $L_{1} \cap \cdots \cap L_{k}$ has dimension $\left.\max \left\{0, \operatorname{dim} L_{1}+\cdots+\operatorname{dim} L_{k}-(k-1) n\right\}\right)$.

For nonempty subsets $F_{1}, \ldots, F_{k} \subset \mathbb{R}^{n}$ we set

$$
\left[F_{1}, \ldots, F_{k}\right]:=\left[L\left(F_{1}\right), \ldots, L\left(F_{k}\right)\right],
$$

where $L\left(F_{i}\right)$ is the linear subspace parallel to the affine hull of $F_{i}(i=1, \ldots, k)$.

Now let polytopes $K_{1}, \ldots, K_{k} \in \mathcal{K}(k \in \mathbb{N})$ be given. For indices $m_{1}, \ldots, m_{k} \in\{1, \ldots, n\}$ satisfying $j:=\sum_{i=1}^{k} m_{i}-(k-1) n \geq 0$ we introduce measures on $\left(\mathbb{R}^{n}\right)^{k}$, the mixed measures $\Phi_{m_{1}, \ldots, m_{k}}^{(j)}\left(K_{1}, \ldots, K_{k} ; \cdot\right)$, by

$$
\begin{aligned}
& \Phi_{m_{1}, \ldots, m_{k}}^{(j)}\left(K_{1}, \ldots, K_{k} ; \cdot\right) \\
&:=\sum_{F_{1} \in \mathcal{F}_{m_{1}}\left(K_{1}\right)} \ldots \sum_{F_{k} \in \mathcal{F}_{m_{k}}\left(K_{k}\right)} \gamma\left(F_{1}, \ldots, F_{k} ; K_{1}, \ldots, K_{k}\right)\left[F_{1}, \ldots, F_{k}\right] \\
& \times \lambda_{F_{1}} \otimes \cdots \otimes \lambda_{F_{k}} .
\end{aligned}
$$


Note that for $k=1$ we have $j=m_{1}$ and hence $\Phi_{m}^{(j)}(K ; \cdot)=\Phi_{m}(K ; \cdot)$.

We can use essentially the same integration technique as sketched for the proof of Theorem 2.3, or that theorem combined with induction, to obtain the following iterated translative formulae for polytopes $K_{1}, \ldots, K_{k} \in \mathcal{K}(k \geq 2)$ and Borel sets $A_{1}, \ldots, A_{k} \in \mathcal{B}\left(\mathbb{R}^{n}\right)$ :

$$
\begin{array}{r}
\int_{\mathbb{R}^{n}} \ldots \int_{\mathbb{R}^{n}} \Phi_{j}\left(K_{1} \cap x_{2} K_{2} \cap \cdots \cap x_{k} K_{k}, A_{1} \cap x_{2} A_{2} \cap \cdots \cap x_{k} A_{k}\right) \\
\times \lambda_{n}\left(\mathrm{~d} x_{2}\right) \cdots \lambda_{n}\left(\mathrm{~d} x_{k}\right) \\
=\sum_{\substack{m_{1}, \ldots, m_{k}=j \\
m_{1}+\cdots+m_{k}=(k-1) n+j}}^{n} \Phi_{m_{1}, \ldots, m_{k}}^{(j)}\left(K_{1}, \ldots, K_{k} ; A_{1} \times \cdots \times A_{k}\right) .
\end{array}
$$

This formula is equivalent to the validity of the equation

$$
\begin{array}{r}
\int_{\left(\mathbb{R}^{n}\right)^{k-1}} \int_{\mathbb{R}^{n}} g\left(y, y-x_{2}, \ldots, y-x_{k}\right) \Phi_{j}\left(K_{1} \cap x_{2} K_{2} \cap \cdots \cap x_{k} K_{k}, \mathrm{~d} y\right) \\
\times \lambda_{n}^{k-1}\left(\mathrm{~d}\left(x_{2}, \ldots, x_{k}\right)\right) \\
=\sum_{\substack{m_{1}, \ldots, m_{k}=j \\
m_{1}+\cdots+m_{k}=(k-1) n+j}}^{n} \int_{\left(\mathbb{R}^{n}\right)^{k}} g\left(x_{1}, x_{2}, \ldots, x_{k}\right) \\
\Phi_{m_{1}, \ldots, m_{k}}^{(j)}\left(K_{1}, \ldots, K_{k} ; \mathrm{d}\left(x_{1}, \ldots, x_{k}\right)\right)
\end{array}
$$

for all nonnegative measurable functions $g$ on $\left(\mathbb{R}^{n}\right)^{k}$. In fact, if the first formula holds, then the second holds for $g=\mathbf{1}_{A_{1} \times \cdots \times A_{k}}$ and thus for elementary functions, hence, by the standard extension, it holds for nonnegative measurable functions. Conversely, if the second formula holds, then the first is true for compact sets $A_{1}, \ldots, A_{k}$, since $\mathbf{1}_{A_{1} \times \cdots \times A_{k}}$ is then the limit of a decreasing sequence of continuous functions. Since both sides of the equation are measures in $A_{1}, \ldots, A_{k}$, the equation holds for Borel sets.

Formula (48) can be extended to general convex bodies, that is, for $K_{1}, \ldots, K_{k} \in \mathcal{K}$ and numbers $k, m_{1}, \ldots, m_{k}, j$ as above, there exist finite measures $\Phi_{m_{1}, \ldots, m_{k}}^{(j)}\left(K_{1}, \ldots, K_{k} ; \cdot\right)$, on $\left(\mathbb{R}^{n}\right)^{k}$, the mixed measures, so that (48) holds. The proof uses approximation of general convex bodies by polytopes, formula (48) for polytopes, and the weak continuity of the curvature measures.

Theorem 3.1 (Iterated translative formula). Let $K_{1}, \ldots, K_{k} \in \mathcal{K}$, where $k \geq 2$, be convex bodies. For $m_{1}, \ldots, m_{k} \in\{1, \ldots, n\}$ with $j:=\sum_{i=1}^{k} m_{i}-$ $(k-1) n \geq 0$, there are finite measures $\Phi_{m_{1}, \ldots, m_{k}}^{(j)}\left(K_{1}, \ldots, K_{k} ; \cdot\right)$ on $\left(\mathbb{R}^{n}\right)^{k}$ such that 


$$
\begin{array}{r}
\int_{\mathbb{R}^{n}} \ldots \int_{\mathbb{R}^{n}} \Phi_{j}\left(K_{1} \cap x_{2} K_{2} \cap \cdots \cap x_{k} K_{k}, A_{1} \cap x_{2} A_{2} \cap \cdots \cap x_{k} A_{k}\right) \\
\times \lambda_{n}\left(\mathrm{~d} x_{2}\right) \cdots \lambda_{n}\left(\mathrm{~d} x_{k}\right) \\
=\sum_{\substack{m_{1}, \ldots, m_{k}=j \\
m_{1}+\cdots+m_{k}=(k-1) n+j}}^{n} \Phi_{m_{1}, \ldots, m_{k}}^{(j)}\left(K_{1}, \ldots, K_{k} ; A_{1} \times \cdots \times A_{k}\right)
\end{array}
$$

for Borel sets $A_{1}, \ldots, A_{k} \in \mathcal{B}\left(\mathbb{R}^{n}\right)$.

We collect some properties of the mixed measures. Most of them can be deduced without difficulty from the corresponding properties of the mixed measures of polytopes, which are obvious.

- Symmetry:

$$
\begin{aligned}
& \Phi_{m_{1}, \ldots, m_{k}}^{(j)}\left(K_{1}, \ldots, K_{k} ; A_{1} \times \cdots \times A_{k}\right) \\
& \quad=\Phi_{m_{\pi(1)}, \ldots, m_{\pi(k)}}^{(j)}\left(K_{\pi(1)}, \ldots, K_{\pi(k)} ; A_{\pi(1)} \times \cdots \times A_{\pi(k)}\right)
\end{aligned}
$$

for each permutation $\pi$ of $\{1, \ldots, k\}$.

- Support property: the support of $\Phi_{m_{1}, \ldots, m_{k}}^{(j)}\left(K_{1}, \ldots, K_{k} ; \cdot\right)$ is contained in $S_{1} \times \cdots \times S_{k}$, where $S_{i}=K_{i}$ if $m_{i}=n$ and $S_{i}=\partial K_{i}$ if $m_{i}<n$.

- Translation covariance: for $t_{1}, \ldots, t_{k} \in \mathbb{R}^{n}$,

$$
\begin{gathered}
\Phi_{m_{1}, \ldots, m_{k}}^{(j)}\left(t_{1} K_{1}, \ldots, t_{k} K_{k} ; t_{1} A_{1} \times \cdots \times t_{k} A_{k}\right) \\
=\Phi_{m_{1}, \ldots, m_{k}}^{(j)}\left(K_{1}, \ldots, K_{k} ; A_{1} \times \cdots \times A_{k}\right) .
\end{gathered}
$$

- Homogeneity: for $\alpha_{1}, \ldots, \alpha_{k} \geq 0$,

$$
\begin{aligned}
& \Phi_{m_{1}, \ldots, m_{k}}^{(j)}\left(\alpha_{1} K_{1}, \ldots, \alpha_{k} K_{k} ; \alpha_{1} A_{1} \times \cdots \times \alpha_{k} A_{k}\right) \\
& \quad=\alpha_{1}^{m_{1}} \cdots \alpha_{k}^{m_{k}} \Phi_{m_{1}, \ldots, m_{k}}^{(j)}\left(K_{1}, \ldots, K_{k} ; A_{1} \times \cdots \times A_{k}\right) .
\end{aligned}
$$

- Weak continuity: the map $\left(K_{1}, \ldots, K_{k}\right) \mapsto \Phi_{m_{1}, \ldots, m_{k}}^{(j)}\left(K_{1}, \ldots, K_{k} ; \cdot\right)$ from $\mathcal{K}^{k}$ into the space of finite measures on $\left(\mathbb{R}^{n}\right)^{k}$ with the weak topology is continuous.

- Additivity: $\Phi_{m_{1}, \ldots, m_{k}}^{(j)}\left(K_{1}, \ldots, K_{k} ; A_{1} \times \cdots \times A_{k}\right)$ is additive in each of its first $k$ arguments.

- Decomposition property:

$$
\begin{aligned}
& \Phi_{m_{1}, \ldots, m_{k-1}, n}^{(j)}\left(K_{1}, \ldots, K_{k} ; A_{1} \times \cdots \times A_{k}\right) \\
& \quad=\Phi_{m_{1}, \ldots, m_{k-1}}^{(j)}\left(K_{1}, \ldots, K_{k-1} ; A_{1} \times \cdots \times A_{k-1}\right) \lambda_{n}\left(K_{k} \cap A_{k}\right)
\end{aligned}
$$

(and similarly for the other arguments, by symmetry). 


\section{- Reduction property:}

$$
\begin{array}{r}
\Phi_{m_{1}, \ldots, m_{k}}^{(j)}\left(K_{1}, \ldots, K_{k} ; A_{1} \times \cdots \times A_{k}\right) \\
=\left(\frac{2}{\kappa_{n-1}}\right)^{j} \frac{1}{j ! \kappa_{j}} \Phi_{m_{1}, \ldots, m_{k}, n-1, \ldots, n-1}^{(0)}(K_{1}, \ldots, K_{k}, \underbrace{B^{n}, \ldots, B^{n}}_{j} ; \\
\left.A_{1} \times \cdots \times A_{k} \times\left(\mathbb{R}^{n}\right)^{j}\right) .
\end{array}
$$

- Local determination: If $K_{1}^{\prime}, \ldots, K_{k}^{\prime} \in \mathcal{K}$ and $A_{i} \subset \operatorname{int} K_{i}^{\prime}$ for $i=$ $1, \ldots, k$, then

$$
\begin{aligned}
& \Phi_{m_{1}, \ldots, m_{k}}^{(j)}\left(K_{1} \cap K_{1}^{\prime}, \ldots, K_{k} \cap K_{k}^{\prime} ; A_{1} \times \cdots \times A_{k}\right) \\
& \quad=\Phi_{m_{1}, \ldots, m_{k}}^{(j)}\left(K_{1}, \ldots, K_{k} ; A_{1} \times \cdots \times A_{k}\right) .
\end{aligned}
$$

The decomposition property has a useful consequence. The condition $m_{1}+$ $\cdots+m_{k}=(k-1) n+j$ implies that at most $n-j$ of the indices $m_{1}, \ldots, m_{k}$ can be smaller than $n$. Hence, all mixed measures with upper index $(j)$ can be expressed in terms of Lebesgue measure and the finitely many mixed measures $\Phi_{m_{1}, \ldots, m_{r}}^{(j)}$, where $r \in\{1, \ldots, n-j\}$. By the reduction property, all mixed measures can further be reduced to the measures $\Phi_{m_{1}, \ldots, m_{k}}^{(0)}$ with $k \in\{1, \ldots, n\}$ and $m_{1}, \ldots, m_{k} \in\{1, \ldots, n-1\}$ satisfying $m_{1}+\cdots+m_{k}=(k-1) n$. The mixed measures with upper index (0) will therefore be considered as basic.

The last of the listed properties, local determination, can be used to extend the definition of the mixed measures, in an obvious way, to closed convex sets that are not necessarily bounded. The iterated translative formula (50) remains valid if the Borel sets $A_{i}$ corresponding to unbounded convex sets $K_{i}$ are bounded.

The total measures

$$
\Phi_{m_{1}, \ldots, m_{k}}^{(j)}\left(K_{1}, \ldots, K_{k} ;\left(\mathbb{R}^{n}\right)^{k}\right)=: V_{m_{1}, \ldots, m_{k}}^{(j)}\left(K_{1}, \ldots, K_{k}\right),
$$

are called the mixed functionals, and those with upper index (0) the basic mixed functionals. In the case of polytopes $K_{1}, \ldots, K_{n}$, the mixed functionals are explicitly given by

$$
\begin{array}{r}
V_{m_{1}, \ldots, m_{k}}^{(j)}\left(K_{1}, \ldots, K_{k}\right) \\
=\sum_{F_{1} \in \mathcal{F}_{m_{1}}\left(K_{1}\right)} \ldots \sum_{F_{k} \in \mathcal{F}_{m_{k}}\left(K_{k}\right)} \gamma\left(F_{1}, \ldots, F_{k} ; K_{1}, \ldots, K_{k}\right)\left[F_{1}, \ldots, F_{k}\right] \\
\times V_{m_{1}}\left(F_{1}\right) \cdots V_{m_{k}}\left(F_{k}\right) .
\end{array}
$$

The mixed measures, and therefore also the mixed functionals, satisfy various integral geometric relations, among them translative formulae, rotation formulae, and Crofton type formulae. 
Hints to the literature. The technical details omitted in this subsection can be found in [50] and [57]. A thorough investigation of the mixed measures, including integral geometric relations and special representations, appears in [59]. In [8], representations of mixed measures in terms of support measures are applied to Boolean models.

Using methods of geometric measure theory, Rataj and Zähle [33] have obtained a general translative formula for support measures of sets with positive reach. An iterated version is proved in [28]. Various extensions and supplements appear in [29], [17], [62], [34], [35]. Translative Crofton formulae for support measures are treated in [30].

\subsection{Basic mixed functionals and support functions}

In this subsection, we will study some special cases of the mixed measures in greater detail and under particular aspects. First, we consider the basic mixed functionals $V_{m_{1}, \ldots, m_{k}}^{(0)}$. They are uniquely determined as the coefficients in the polynomial expansion

$$
\begin{gathered}
\int_{\mathbb{R}^{n}} \ldots \int_{\mathbb{R}^{n}} \chi\left(\alpha_{1} K_{1} \cap x_{2} \alpha_{2} K_{2} \cap \cdots \cap x_{k} \alpha_{k} K_{k}\right) \lambda_{n}\left(\mathrm{~d} x_{2}\right) \cdots \lambda_{n}\left(\mathrm{~d} x_{k}\right) \\
=\sum_{\substack{m_{1}, \ldots, m_{k}=0 \\
m_{1}+\cdots+m_{k}=(k-1) n}}^{n} \alpha_{1}^{m_{1}} \cdots \alpha_{k}^{m_{k}} V_{m_{1}, \ldots, m_{k}}^{(0)}\left(K_{1}, \ldots, K_{k}\right)
\end{gathered}
$$

for $K_{1}, \ldots, K_{k} \in \mathcal{K}, \alpha_{1} \ldots, \alpha_{k} \geq 0, k \geq 2$ (a special case of (50)). Our first aim is to show that the notion of basic mixed functionals can, in a certain sense, be viewed as dual to the notion of mixed volumes, which constitute an important set of functionals in the classical theory of convex bodies. It suffices to consider polytopes (the extension to general convex bodies is achieved by approximation). For these, we will obtain a class of representations of the basic mixed measures of greater generality than their original definition, which is the representation

$$
\begin{aligned}
V_{m_{1}, \ldots, m_{k}}^{(0)}\left(K_{1}, \ldots, K_{k}\right) & \\
=\sum_{F_{1} \in \mathcal{F}_{m_{1}}\left(K_{1}\right)} \ldots \sum_{F_{k} \in \mathcal{F}_{m_{k}}\left(K_{k}\right)} \gamma\left(F_{1}, \ldots,\right. & \left.F_{k} ; K_{1}, \ldots, K_{k}\right)\left[F_{1}, \ldots, F_{k}\right] \\
& \times V_{m_{1}}\left(F_{1}\right) \cdots V_{m_{k}}\left(F_{k}\right) .
\end{aligned}
$$

We describe a general construction leading to different types of mixed functionals. For $k \geq 2$, we consider the Euclidean vector space $X:=\left(\mathbb{R}^{n}\right)^{k}$ (with the scalar product induced from that of $\mathbb{R}^{n}$ ), together with a surjective linear map $f: X \rightarrow \mathbb{R}^{q}$ onto a Euclidean space $\mathbb{R}^{q}$, where $q<k n$. For $L:=(\operatorname{ker} f)^{\perp}$, the orthogonal complement of the kernel of $f$, let $\pi_{L}: X \rightarrow L$ 
denote the orthogonal projection. If $\tilde{f}:=f \mid L$, then $f=\tilde{f} \circ \pi_{L}$, and $\tilde{f}: L \rightarrow \mathbb{R}^{q}$ is an isomorphism.

For a given polytope $P \subset X$, let $\mathcal{G}_{v}(P)$ be a set of $q$-dimensional faces of $P$ with the property that, for the induced Lebesgue measure $\Lambda_{L}$ in $L$,

$$
\Lambda_{L}\left(\pi_{L} P\right)=\sum_{F \in \mathcal{G}_{v}(P)} \Lambda_{L}\left(\pi_{L} F\right)
$$

and hence

$$
V_{q}(f P)=\sum_{F \in \mathcal{G}_{v}(P)} V_{q}(f F) .
$$

Such sets $\mathcal{G}_{v}(P)$, depending on a parameter $v$, can be obtained as follows. We choose a vector $v \in L^{\perp} \backslash\{0\}$ satisfying

$$
\operatorname{dim} F(P, u) \leq q \quad \text { for all } u \in L_{v}^{+}:=L+\{\alpha v: \alpha>0\}
$$

$(F(P, u)$ denotes the face of $P$ with exterior normal vector $u)$. The condition (55) excludes only vectors from finitely many proper linear subspaces of $L^{\perp}$. With this choice, the set

$$
\mathcal{G}_{v}(P):=\left\{F \in \mathcal{F}_{q}(P): N(P, F) \cap L_{v}^{+} \neq \emptyset\right\}
$$

will satisfy (53). This is seen if one decomposes the projection $\pi_{L}$ into the orthogonal projection $\pi_{L, v}$ on to $\operatorname{lin}(L \cup\{v\})$, followed by the orthogonal projection from this space to L. In fact, let $\mathcal{S}_{q}$ be the set of $q$-faces of $\pi_{L, v} P$ having a normal vector in $L_{v}^{+}$. The images of these $q$-faces under projection to $L$ cover $\pi P$ without overlappings. On the other hand, under $\pi_{L, v}$, the set $\mathcal{G}_{v}(P)$ is in one-to-one correspondence with the set $\mathcal{S}_{q}$. This proves (53).

Now let $P_{1}, \ldots, P_{k} \subset \mathbb{R}^{n}$ be polytopes, and let $P:=P_{1} \times \cdots \times P_{k}$. Every $q$-face $F$ of $P$ is of the form $F=F_{1} \times \cdots \times F_{k}$ with faces $F_{i} \in \mathcal{F}_{m_{i}}\left(P_{i}\right)$ $(i=1, \ldots, k)$ for suitable $m_{1}, \ldots, m_{k} \in\{0, \ldots, n\}$ satisfying $m_{1}+\cdots+m_{k}=q$. For $\alpha_{1}, \ldots, \alpha_{k} \geq 0$, we clearly have

$$
\begin{aligned}
& V_{q}\left(f\left(\alpha_{1} F_{1} \times \cdots \times \alpha_{k} F_{k}\right)\right) \\
& \quad=\alpha_{1}^{m_{1}} \cdots \alpha_{k}^{m_{k}} c\left(f, F_{1}, \ldots, F_{k}\right) V_{m_{1}}\left(F_{1}\right) \cdots V_{m_{k}}\left(F_{k}\right),
\end{aligned}
$$

where $c\left(f, F_{1}, \ldots, F_{k}\right)$ is the factor by which the linear map $f \mid \operatorname{aff}\left(F_{1} \times \cdots \times F_{k}\right)$ changes the $q$-dimensional volume. Together with (54), this gives

$$
\begin{aligned}
V_{q} & \left(f\left(\alpha_{1} P_{1} \times \cdots \times \alpha_{k} P_{k}\right)\right) \\
\quad= & \sum_{\substack{m_{1}, \ldots, m_{k}=0 \\
m_{1}+\cdots, m_{k}=q}}^{n} \alpha_{1}^{m_{1}} \cdots \alpha_{k}^{m_{k}} V_{m_{1}, \ldots, m_{k}}^{f}\left(P_{1}, \ldots, P_{k}\right)
\end{aligned}
$$

with 


$$
\begin{aligned}
& V_{m_{1}, \ldots, m_{k}}^{f}\left(P_{1}, \ldots, P_{k}\right) \\
& =\sum_{\substack{F_{i} \in \mathcal{F}_{m_{i}}\left(P_{i}\right), i=1, \ldots, k \\
F_{1} \times \cdots \times F_{k} \in \mathcal{G}_{v}\left(P_{1} \times \cdots \times P_{k}\right)}} c\left(f, F_{1}, \ldots, F_{k}\right) V_{m_{1}}\left(F_{1}\right) \cdots V_{m_{k}}\left(F_{k}\right) . \\
&
\end{aligned}
$$

Here $v$ is chosen according to (55), for $P=P_{1} \times \cdots \times P_{k}$. The expansion (56) determines the coefficients $V_{m_{1}, \ldots, m_{k}}^{f}\left(P_{1}, \ldots, P_{k}\right)$ uniquely; hence they do not depend on the choice of the set $\mathcal{G}_{v}\left(P_{1} \times \cdots \times P_{k}\right)$. These coefficients represent a general type of mixed functionals, depending on the choice of the number $k$ and the linear map $f$.

For a concrete example, let $f:\left(\mathbb{R}^{n}\right)^{k} \rightarrow \mathbb{R}^{n}$ be defined by $f\left(x_{1}, \ldots, x_{n}\right):=$ $x_{1}+\cdots+x_{n}$. Then

$$
f\left(\alpha_{1} P_{1} \times \cdots \times \alpha_{k} P_{k}\right)=\alpha_{1} P_{1}+\cdots+\alpha_{k} P_{k},
$$

and (56) gives

$$
\begin{aligned}
& V_{n}\left(\alpha_{1} P_{1}+\cdots+\alpha_{k} P_{k}\right) \\
& \quad=\sum_{\substack{m_{1}, \ldots, m_{k}=0 \\
m_{1}+\cdots+m_{k}=n}}^{n} \alpha_{1}^{m_{1}} \cdots \alpha_{k}^{m_{k}} V_{m_{1}, \ldots, m_{k}}^{f}\left(P_{1}, \ldots, P_{k}\right) .
\end{aligned}
$$

This shows that

$$
V_{m_{1}, \ldots, m_{k}}^{f}\left(P_{1}, \ldots, P_{k}\right)=\left(\begin{array}{c}
n \\
m_{1}, \ldots, m_{k}
\end{array}\right) V(\underbrace{P_{1}, \ldots, P_{1}}_{m_{1}}, \ldots, \underbrace{P_{k}, \ldots, P_{k}}_{m_{k}}),
$$

where $V: \mathcal{K}^{n} \rightarrow \mathbb{R}$ denotes the mixed volume, known from the theory of convex bodies. Equation (57) provides a class of special representations for the mixed volumes of polytopes.

In the second example, let $g:\left(\mathbb{R}^{n}\right)^{k} \rightarrow\left(\mathbb{R}^{n}\right)^{k-1}$ be defined by $g\left(y_{1}, \ldots, y_{k}\right)$ $:=\left(y_{1}-y_{2}, \ldots, y_{1}-y_{k}\right)$. Then

$$
g\left(P_{1} \times \cdots \times P_{k}\right)=\left\{\left(x_{2}, \ldots, x_{k}\right) \in\left(\mathbb{R}^{n}\right)^{k-1}: P_{1} \cap x_{2} P_{2} \cap \cdots \cap x_{k} P_{k} \neq \emptyset\right\},
$$

hence

$$
\begin{aligned}
& V_{(k-1) n}\left(g\left(\alpha_{1} P_{1} \times \cdots \times \alpha_{k} P_{k}\right)\right) \\
& \quad=\int_{\mathbb{R}^{n}} \cdots \int_{\mathbb{R}^{n}} \chi\left(\alpha_{1} P_{1} \cap x_{2} \alpha_{2} P_{2} \cap \cdots \cap x_{k} \alpha_{k} P_{k}\right) \lambda_{n}\left(\mathrm{~d} x_{2}\right) \cdots \lambda_{n}\left(\mathrm{~d} x_{k}\right) .
\end{aligned}
$$

Now (56) shows that

$$
V_{m_{1}, \ldots, m_{k}}^{g}\left(P_{1}, \ldots, P_{k}\right)=V_{m_{1}, \ldots, m_{k}}^{(0)}\left(P_{1}, \ldots, P_{k}\right)
$$

thus we obtain the basic mixed functionals. The constructions leading to mixed volumes and to basic mixed functionals can be considered as duals of 
each other, since the kernels of the employed maps $f$ and $g$ are complementary orthogonal subspaces of $\left(\mathbb{R}^{n}\right)^{k}$.

In order to make the representation (57) more explicit in the concrete cases, one has to translate condition (55) to the actual situation and to determine the factors $c\left(f, F_{1}, \ldots, F_{k}\right)$. We give here the result only for the second example. In that case, we say that a vector $w \in \mathbb{R}^{n}$ is admissible for the polytopes $P_{1}, \ldots, P_{k}$ if

$$
w \notin N\left(P_{1}, F_{1}\right)+\cdots+N\left(P_{k}, F_{k}\right)
$$

whenever $F_{1} \in \mathcal{F}_{m_{1}}\left(P_{1}\right), \ldots, F_{k} \in \mathcal{F}_{m_{k}}\left(P_{k}\right)$ with numbers $m_{1}, \ldots, m_{k} \in$ $\{1, \ldots, n\}$ satisfying $m_{1}+\cdots+m_{k}=(k-1) n+1$. Then we get the following result. If $w$ is admissible for $P_{1}, \ldots, P_{k}$, then

$$
\begin{aligned}
V_{m_{1}, \ldots, m_{k}}^{(0)}\left(P_{1}, \ldots, P_{k}\right) & \\
=\sum_{F_{1} \in \mathcal{F}_{m_{1}}\left(P_{1}\right)} \cdots & \sum_{\substack{F_{k} \in \mathcal{F}_{m_{k}}\left(P_{k}\right) \\
\times\left[F_{1}, \ldots, F_{k}\right] V_{m_{1}}\left(F_{1}\right) \cdots V_{m_{k}}\left(F_{k}\right) .}} \mathbf{1}_{N\left(P_{1}, F_{1}\right)+\cdots+N\left(P_{k}, F_{k}\right)}(w)
\end{aligned}
$$

This representation is more general than (52). Equation (58) holds for all vectors $w \in S^{n-1}$, with the exception of those in finitely many great subspheres (depending on $P_{1}, \ldots, P_{k}$ ). Integration over $S^{n-1}$ with respect to spherical Lebesgue measure $\sigma_{n-1}$ yields (52).

Our second aim in this subsection is the presentation of a set-valued analogue of the iterated translative formula (51), of the form

$$
\begin{gathered}
\int_{\mathbb{R}^{n}} \ldots \int_{\mathbb{R}^{n}}\left(\alpha_{1} K_{1} \cap x_{2} \alpha_{2} K_{2} \cap \cdots \cap x_{k} \alpha_{k} K_{k}\right) \lambda_{n}\left(\mathrm{~d} x_{2}\right) \cdots \lambda_{n}\left(\mathrm{~d} x_{k}\right) \\
=\sum_{\substack{m_{1}, \ldots, m_{k}=0 \\
m_{1}+\cdots+m_{k}=(k-1) n+1}}^{n} \alpha_{1}^{m_{1}} \cdots \alpha_{k}^{m_{k}} T_{m_{1}, \ldots, m_{k}}\left(K_{1}, \ldots, K_{k}\right)+t,
\end{gathered}
$$

with convex bodies $T_{m_{1}, \ldots, m_{k}}\left(K_{1}, \ldots, K_{k}\right)$ and a translation vector $t$. The integral of a function with values in $\mathcal{K}$ is defined via support functions, so that this formula is equivalent to a relation for support functions.

It is convenient in the following to use the centred support function $h^{*}$. This is the support function with respect to the Steiner point $s$, thus

$$
h^{*}(K, u)=h(K-s(K), u)=h(K, u)-\langle s(K), u\rangle,
$$

where the Steiner point of the convex body $K$ is defined by

$$
s(K):=\frac{1}{\kappa_{n}} \int_{S^{n-1}} h(K, u) u \sigma_{n-1}(\mathrm{~d} u) .
$$

The centred support function is related to a special case of the mixed measures. We recall from Subsection 3.1 that the mixed measures can be 
extended to unbounded closed convex sets. The special representations for polytopes extend to polyhedral sets. We use this for the sets

$$
u^{+}:=\left\{x \in \mathbb{R}^{n}:\langle x, u\rangle \geq 0\right\}, \quad u^{\perp}:=\left\{x \in \mathbb{R}^{n}:\langle x, u\rangle=0\right\},
$$

where $u \in S^{n-1}$. Choosing a Borel set $A(u) \subset u^{\perp}$ with $\lambda_{n-1}(A(u))=1$, we have, for polytopes $P$,

$$
\begin{aligned}
h^{*}(P, u) & =\sum_{F \in \mathcal{F}_{1}(P)} \gamma\left(F, u^{\perp} ; P, u^{+}\right)\left[F, u^{\perp}\right] V_{1}(F) \\
& =\Phi_{1, n-1}^{(0)}\left(P, u^{+} ; \mathbb{R}^{n} \times A(u)\right) .
\end{aligned}
$$

To prove (60), we first remark that, for a polytope $P$,

$$
\sum_{e \in \mathcal{F}_{0}(P)} \gamma(e, P) e=s(P) \quad \sum_{e \in \mathcal{F}_{0}(P)} \gamma(e, P)=\chi(P)=1,
$$

where, for the ease of notation, we do not distinguish between a vertex $e$ and the corresponding 0 -face $\{e\}$ of $P$. Let

$$
H_{u, t}^{+}:=\left\{x \in \mathbb{R}^{n}:\langle x, u\rangle \geq t\right\}
$$

for $u \in S^{n-1}$ and $t \in \mathbb{R}$, and choose a number $c$ satisfying $P \subset H_{u, c}^{+}$. Then

$$
h(P, u)-c=\int_{c}^{\infty} \chi\left(P \cap H_{u, t}^{+}\right) \mathrm{d} t .
$$

Using (62), we get

$$
\begin{aligned}
\int_{c}^{\infty} & \chi\left(P \cap H_{u, t}^{+}\right) \mathrm{d} t \\
= & \int_{c}^{\infty} \sum_{e \in \mathcal{F}_{0}\left(P \cap H_{u, t}^{+}\right)} \gamma\left(e, P \cap H_{u, t}^{+}\right) \mathrm{d} t \\
= & \int_{c}^{\infty} \sum_{e \in \mathcal{F}_{0}(P)} \gamma(e, P) \mathbf{1}\{\langle e, u\rangle \geq t\} \mathrm{d} t \\
& +\int_{c}^{\infty} \sum_{F \in \mathcal{F}_{1}(P)} \gamma\left(F, u^{\perp} ; P, u^{+}\right) \chi\left(F \cap H_{u, t}\right) \mathrm{d} t \\
= & \sum_{e \in \mathcal{F}_{0}(P)} \gamma(e, P)(\langle e, u\rangle-c)+\sum_{F \in \mathcal{F}_{1}(P)} \gamma\left(F, u^{\perp} ; P, u^{+}\right)\left[F, u^{\perp}\right] V_{1}(F) \\
= & \langle s(P), u\rangle-c+\sum_{F \in \mathcal{F}_{1}(P)} \gamma\left(F, u^{\perp} ; P, u^{+}\right)\left[F, u^{\perp}\right] V_{1}(F),
\end{aligned}
$$

which together with (63) proves (60). 
As mentioned earlier, the mixed measures, which arose from an iterated translative formula, satisfy themselves an iterated formula. We can apply this to the mixed measure (61), or else we can use essentially the same method that led to the iterated formula (48). In either way, the following theorem can be obtained.

Theorem 3.2. If $P_{1}, \ldots, P_{k} \subset \mathbb{R}^{n}$ are polytopes and $\alpha_{1}, \ldots, \alpha_{k} \geq 0(k \geq 2)$, then

$$
\begin{gathered}
\int_{\mathbb{R}^{n}} \ldots \int_{\mathbb{R}^{n}} h^{*}\left(\alpha_{1} P_{1} \cap x_{2} \alpha_{2} P_{2} \cap \cdots \cap x_{k} \alpha_{k} P_{k}, \cdot\right) \lambda_{n}\left(\mathrm{~d} x_{2}\right) \cdots \lambda_{n}\left(\mathrm{~d} x_{k}\right) \\
=\sum_{\substack{m_{1}, \ldots, m_{k}=0 \\
m_{1}+\cdots+m_{k}=(k-1) n+1}}^{n} \alpha_{1}^{m_{1}} \cdots \alpha_{k}^{m_{k}} h_{m_{1}, \ldots, m_{k}}^{*}\left(P_{1}, \ldots, P_{k} ; \cdot\right)
\end{gathered}
$$

with

$$
\begin{array}{r}
h_{m_{1}, \ldots, m_{k}}^{*}\left(P_{1}, \ldots, P_{k} ; u\right) \\
:=\sum_{F_{1} \in \mathcal{F}_{m_{1}}\left(P_{1}\right)} \ldots \sum_{\begin{array}{c}
F_{k} \in \mathcal{F}_{m_{k}}\left(P_{k}\right) \\
\end{array}} \gamma\left(F_{1}, \ldots, F_{k}, u^{\perp} ; P_{1}, \ldots, P_{k}, u^{+}\right) \\
\quad \times\left[F_{1}, \ldots, F_{k}, u^{\perp}\right] V_{m_{1}}\left(F_{1}\right) \cdots V_{m_{k}}\left(F_{k}\right)
\end{array}
$$

for $u \in S^{n-1}$.

The left-hand side of (64) is clearly a support function. It is, at the moment, not clear that each summand on the right-hand side is also a support function. To prove that this holds true, we need a class of more general representations of the functions $h_{m_{1}, \ldots, m_{k}}^{*}\left(P_{1}, \ldots, P_{k} ; \cdot\right)$. These can be obtained by methods similar to those employed previously in this subsection. Let $u \in \mathbb{R}^{n} \backslash\{0\}$. We say that the vector $w \in \mathbb{R}^{n}$ is admissible for $\left(P_{1}, \ldots, P_{k}, u\right)$ if it is admissible for $\left(P_{1}, \ldots, P_{k}\right)$ and if

$$
w \notin N\left(P_{1}, F_{1}\right)+\cdots+N\left(P_{k}, F_{k}\right)+\operatorname{pos}\{-u\}
$$

(where pos denotes the positive hull) whenever $F_{1} \in \mathcal{F}_{m_{1}}\left(P_{1}\right), \ldots, F_{k} \in$ $\mathcal{F}_{m_{k}}\left(P_{k}\right)$ for numbers $m_{1}, \ldots, m_{k} \in\{1, \ldots, n\}$ satisfying $m_{1}+\cdots+m_{k}=$ $(k-1) n+2$. If $w \in \mathbb{R}^{n}$ is admissible for $\left(P_{1}, \ldots, P_{k}, u\right)$, it can be deduced from (60) and (58) that

$$
\begin{aligned}
& h_{m_{1}, \ldots, m_{k}}^{*}\left(P_{1}, \ldots, P_{k} ; u\right) \\
& =\left\langle v_{m_{1}, \ldots, m_{k}}\left(P_{1}, \ldots, P_{k}, w\right), u\right\rangle \\
& \quad+\sum_{F_{1} \in \mathcal{F}_{m_{1}}\left(P_{1}\right)} \ldots \sum_{F_{k} \in \mathcal{F}_{m_{k}}\left(P_{k}\right)} \mathbf{1}_{N\left(P_{1}, F_{1}\right)+\cdots+N\left(P_{k}, F_{k}\right)+\operatorname{pos}\{-u\}}(w) \\
& \quad \times\|u\|\left[F_{1}, \ldots, F_{k}, u^{\perp}\right] V_{m_{1}}\left(F_{1}\right) \cdots V_{m_{k}}\left(F_{k}\right)
\end{aligned}
$$


for $u \in \mathbb{R}^{n}$. By $v_{m_{1}, \ldots, m_{k}}\left(P_{1}, \ldots, P_{k}, w\right)$ we have denoted a vector which could be given explicitly. The point now is that every $u_{0} \in \mathbb{R}^{n} \backslash\{0\}$ has a convex neighbourhood $U$ with the following properties: there is a vector $w$ that is admissible for $\left(P_{1}, \ldots, P_{k}, u\right)$, for all $u \in U$, and the function

$$
u \mapsto \mathbf{1}_{N\left(P_{1}, F_{1}\right)+\cdots+N\left(P_{k}, F_{k}\right)+\operatorname{pos}\{-u\}}(w)
$$

is constant for $u \in U$. This implies that the right-hand side of (67) defines a convex function of $u \in U$. Hence, the function $h_{m_{1}, \ldots, m_{k}}^{*}\left(P_{1}, \ldots, P_{k} ; \cdot\right)$ is locally a support function and therefore also globally. The convex body determined by this support function is denoted by $T_{m_{1}, \ldots, m_{k}}\left(P_{1}, \ldots, P_{k}\right)$. With this definition, (59) holds for polytopes, and an extension to general convex bodies can be achieved by approximation.

Hints to the literature. The general type of mixed functionals, of which the mixed volumes and the basic mixed functionals are special cases, was introduced in [43]; the representation (58) is found there. Theorem 3.2 was first obtained in [58]. Also formula (60) appears there. The simpler proof of the latter, as given here, and the general representation (67), are in [47]. The convexity of the functions $h_{m_{1}, \ldots, m_{k}}^{*}\left(P_{1}, \ldots, P_{k} ; \cdot\right)$, and thus formula (59) with convex bodies $T_{m_{1}, \ldots, m_{k}}\left(K_{1}, \ldots, K_{k}\right)$, was first proved in [9] (for $k=2$ ); the simpler proof sketched here is carried out in [47]. McMullen [26] has developed a general theory of 'mixed fibre polytopes', of which the mixed polytopes $T_{m_{1}, \ldots, m_{k}}\left(P_{1}, \ldots, P_{k}\right)$ are a special case.

\subsection{Further topics of translative integral geometry}

We briefly mention further results of translative integral geometry. First, the elementary formula (47),

$$
\int_{\mathbb{R}^{n}} \chi(K \cap t M) \lambda_{n}(\mathrm{~d} t)=\sum_{i=0}^{n}\left(\begin{array}{c}
n \\
i
\end{array}\right) V_{i}\left(K, M^{*}\right),
$$

has counterparts where one or both of the bodies are replaced by their boundaries. This fact is highly non-elementary. One reason is that the boundary of a general convex body need not even be a set with positive reach, another is the observation that the intersection of the boundary of a convex body with another convex body need not have finite Euler characteristic (for which now a topological definition is needed). Moreover, approximation by polytopes (for which the results are easy) cannot be applied. Nevertheless, the following has been proved. If $K, M \in \mathcal{K}$ are $n$-dimensional convex bodies, then

$$
\begin{aligned}
\int_{\mathbb{R}^{n}} \chi(\partial K \cap t M) \mathrm{d} t \\
\quad=\sum_{i=0}^{n-1}\left(\begin{array}{c}
n \\
i
\end{array}\right)\left\{V_{i}\left(K, M^{*}\right)+(-1)^{n-i-1} V_{i}(K, M)\right\}
\end{aligned}
$$


and

$$
\begin{aligned}
& \int_{\mathbb{R}^{n}} \chi(\partial K \cap t \partial M) \mathrm{d} t \\
& \quad=\left(1+(-1)^{n}\right) \sum_{i=0}^{n-1}\left(\begin{array}{c}
n \\
i
\end{array}\right)\left\{V_{i}\left(K, M^{*}\right)+(-1)^{i-1} V_{i}(K, M)\right\} .
\end{aligned}
$$

From these formulae one can deduce, by additional integrations over the rotation group, the kinematic formulae

$$
\begin{aligned}
& \int_{G_{n}} \chi(\partial K \cap g M) \mu(\mathrm{d} g) \\
& \quad=\sum_{k=0}^{n-1}\left(1-(-1)^{n-k}\right) c_{0, n}^{k, n-k} V_{k}(K) V_{n-k}(M)
\end{aligned}
$$

and

$$
\begin{aligned}
& \int_{G_{n}} \chi(\partial K \cap g \partial M) \mu(\mathrm{d} g) \\
& \quad=\left(1+(-1)^{n}\right) \sum_{k=0}^{n-1}\left(1-(-1)^{k}\right) c_{0, n}^{k, n-k} V_{k}(K) V_{n-k}(M) .
\end{aligned}
$$

Our second topic in this subsection is motivated by Hadwiger's general integral geometric theorem (Theorem 1.4). It provides an abstract version of the principal kinematic formula, holding for arbitrary continuous valuations (additive functions). One may ask whether a similarly general result holds in translative integral geometry. However, this can hardly be expected. What can be achieved, is an analogous result for continuous valuations that are simple. A valuation on $\mathcal{K}$ is simple if it is zero on convex bodies of dimension less than $n$.

The following theorem involves, besides the support function $h(K, \cdot)$ of a convex body $K$, also its area measure $S_{n-1}(K, \cdot)=2 \Xi_{n-1}\left(K, \mathbb{R}^{n} \times \cdot\right)$. The geometric meaning of this measure is as follows. For a Borel subset $A$ of the unit sphere $S^{n-1}$, the value $S_{n-1}(K, A)$ is the area (the $(n-1)$-dimensional Hausdorff measure) of the set of boundary points of $K$ at which there exists an outer unit normal vector in $A$.

Theorem 3.3. Let $\varphi: \mathcal{K} \rightarrow \mathbb{R}$ be a continuous simple valuation. Then

$$
\int_{\mathbb{R}^{n}} \varphi(K \cap x M) \lambda_{n}(\mathrm{~d} x)=\varphi(K) V_{n}(M)+\int_{S^{n-1}} f_{K, \varphi}(u) S_{n-1}(M, \mathrm{~d} u)
$$

for $K, M \in \mathcal{K}$, where $f_{K, \varphi}: S^{n-1} \rightarrow \mathbb{R}$ is the odd function given by 


$$
f_{K, \varphi}(u)=-\varphi(K) h(K, u)+\int_{-h(K,-u)}^{h(K, u)} \varphi\left(K \cap H^{-}(u, t)\right) \mathrm{d} t .
$$

Hints to the literature. The kinematic formulae (70) und (71) were conjectured by Firey (see Problem 18 in the collection [11]). A proof of the more general formulae (68) und (69), and thus of (70) und (71), was given in [21]. In [20], these integral geometric results were extended to lower-dimensional sets, and iterated formulae were established; these were applied to stochastic geometry. Theorem 3.3 was proved in [48].

\section{Intersection Formulae without Invariance}

If $M \in \mathcal{K}$ is a $k$-dimensional convex body, $k \in\{1, \ldots, n-1\}$, the case $j=0$ of the Crofton formula (15) reduces to

$$
\int_{\mathcal{E}_{n-k}^{n}} \operatorname{card}(M \cap E) \mu_{n-k}(\mathrm{~d} E)=\frac{\kappa_{k} \kappa_{n-k}}{\left(\begin{array}{l}
n \\
k
\end{array}\right) \kappa_{n}} \lambda_{k}(M),
$$

since $V_{0}(M \cap E)=\operatorname{card}(M \cap E)$ for $\mu_{n-k}$-almost all $(n-k)$-planes $E$ (here card denotes the number of elements, possibly $\infty$ ), and $V_{k}(M)=\lambda_{k}(M)$. Formula (72) remains true if $M$ is a $k$-dimensional compact $C^{1}$ submanifold of $\mathbb{R}^{n}$ (or, more generally, a $\left(\mathcal{H}^{k}, k\right)$-rectifiable Borel set) and $\lambda_{k}$ denotes the $k$-dimensional Euclidean surface area. Relations of this type are also known as Crofton formulae. They provide beautiful integral geometric interpretations of $k$-dimensional areas.

In this section, we study such Crofton formulae from a more general and 'reverse' point of view: given a notion of $k$-dimensional area that replaces $\lambda_{k}$, does there exist a measure on the space $\mathcal{E}_{n-k}^{n}$ of $(n-k)$-planes so that a counterpart to (72) is valid for a sufficiently large class of $k$-dimensional surfaces $M$ ? The measures on $\mathcal{E}_{n-k}^{n}$ we are seeking are always locally finite Borel measures. We shall admit signed measures as well, but we distinguish clearly between the cases of positive and of signed measures. Invariance properties of these measures are only postulated to the extent that the considered areas are themselves invariant. In the first subsection, areas and measures will be translation invariant, but no invariance property is assumed in the second subsection.

The third subsection is devoted to a special topic from stochastic geometry, the Poisson hyperplane processes. The connection with the Crofton formulae treated before will not be that of an application, but will rather consist in a common structural background. A basic feature in both studies is a relation between certain measures on the space of hyperplanes and special convex bodies, the (generalized) zonoids. Another common feature is the generation of lower dimensional flats as intersections of hyperplanes, and a corresponding generation of measures. 


\subsection{Minkowski Spaces}

Our first topic are Crofton formulae in Minkowski geometry. A Minkowski space is (here) a finite dimensional normed space, say $\left(\mathbb{R}^{n},\|\cdot\|\right)$. The unit ball of this space,

$$
B:=\left\{x \in \mathbb{R}^{n}:\|x\| \leq 1\right\},
$$

is a convex body with 0 as interior point and centre of symmetry. The space of such convex bodies will be denoted by $\mathcal{C}^{n}$. The norm $\|\cdot\|$ induces a metric $d$ by $d(x, y):=\|x-y\|$ for $x, y \in \mathbb{R}^{n}$.

For the subsequent computations, it is convenient to retain also the Euclidean structure on $\mathbb{R}^{n}$ given by the scalar product $\langle\cdot, \cdot\rangle$, although we work in a Minkowski space with norm $\|\cdot\|$. This 'impure' procedure simplifies calculations and presentation.

The metric $d$ induces, in a well-known way, a notion of curve length for rectifiable curves. This curve length is invariant under translations. We denote the Minkowskian length by $\operatorname{vol}_{1}$; thus, in particular, $\operatorname{vol}_{1}(S)=\|a-b\|$ if $S$ is the segment with endpoints $a, b$.

In contrast to the case $k=1$, where the metric induces a natural notion of curve length, for $k>1$ there is no canonical notion of a $k$-dimensional area in Minkowski spaces, but rather a variety of options. The principal ambiguity can be made clear in the case $k=n$, the case of a notion of volume. A reasonable notion of volume in the Minkowski space $\left(\mathbb{R}^{n},\|\cdot\|\right)$ should be a Borel measure, assigning a positive finite value to every nonempty bounded open set, and determined solely by the Minkowskian metric. For the latter reason, it should be invariant under Minkowskian isometries and thus, in particular, under translations. The theory of Haar measures tells us that such a measure is unique up to a positive constant factor, and thus it is a constant multiple of the Lebesgue measure $\lambda_{n}$ induced by the chosen Euclidean structure. Thus, a Minkowskian notion of volume in $\left(\mathbb{R}^{n},\|\cdot\|\right)$ is fixed if we assign a value of this volume to the unit ball $B$. Since the notion of volume should be the same in isometric Minkowski spaces, the value assigned to $B$ should depend only on the equivalence class of $B$ under linear transformations. Thus, choosing a notion of Minkowskian volume for $n$-dimensional Minkowski spaces is equivalent to choosing a positive real function $\alpha_{n}$ on the space $\mathcal{C}^{n}$ which is invariant under linear transformations of $\mathbb{R}^{n}$. If $\alpha_{n}$ is chosen, then in the Minkowski space with unit ball $B$ the induced volume $\alpha_{n, B}$ is given by

$$
\alpha_{n, B}(M)=\frac{\alpha_{n}(B)}{\lambda_{n}(B)} \lambda_{n}(M)
$$

for $M \in \mathcal{B}\left(\mathbb{R}^{n}\right)$ (recall that $\mathcal{B}(X)$ is the $\sigma$-algebra of Borel sets of the topological space $X)$. This convenient representation does not depend on the choice of the Euclidean structure.

The choice just of a normalizing factor may seem rather unimportant, but it is not. Different choices make essential differences if we now employ this 
procedure for the definition of lower dimensional areas. Let $k \in\{1, \ldots, n\}$. For a $k$-dimensional convex body $M$, we denote by $L_{M}$ the linear subspace of $\mathbb{R}^{n}$ parallel to the affine hull of $M$. Since a Minkowskian $k$-area is assumed to be translation invariant, its value at $M$ should depend only on the Minkowski metric in the subspace $L_{M}$. The unit ball of this Minkowski space is $B \cap L_{M}$. This leads us to the following axioms for a Minkowskian $k$-area. Let $\mathcal{C}^{k}$ denote the set of $k$-dimensional convex bodies in $\mathbb{R}^{n}$ which are centrally symmetric with respect to 0 . A $k$-normalizing function is a function $\alpha_{k}: \mathcal{C}^{k} \rightarrow \mathbb{R}^{+}$ which is (i) continuous, (ii) invariant under linear transformations, and (iii) satisfies $\alpha_{k}\left(E^{k}\right)=\kappa_{k}$ if $E^{k}$ is a $k$-dimensional ellipsoid. Such a function induces, in a Minkowski space $\left(\mathbb{R}^{n},\|\cdot\|\right)$ with unit ball $B$, a Minkowskian $k$-area $\alpha_{k, B}$ by

$$
\alpha_{k, B}(M):=\frac{\alpha_{k}\left(B \cap L_{M}\right)}{\lambda_{k}\left(B \cap L_{M}\right)} \lambda_{k}(M)
$$

for every $k$-dimensional convex body $M$. Again, this is independent of the Euclidean structure. The axiom (i) for a $k$-normalizing function seems reasonable, (ii) ensures the invariance of the $k$-area under Minkowskian isometries, and (iii) is assumed in order to obtain the standard $k$-area if the space is Euclidean. The Minkowskian $k$-area can be extended to more general sets, for example to $k$-dimensional $C^{1}$-submanifolds $M$, by

$$
\alpha_{k, B}(M)=\int_{M} \frac{\alpha_{k}\left(B \cap T_{x} M\right)}{\lambda_{k}\left(B \cap T_{x} M\right)} \lambda_{k}(\mathrm{~d} x),
$$

where $T_{x} M$ denotes the tangent space of $M$ at $x$, considered as a subspace of $\mathbb{R}^{n}$.

For the quotient appearing in the integrand, we use the notation

$$
\frac{\alpha_{k}(B \cap L)}{\lambda_{k}(B \cap L)}=: \sigma_{k, B}(L) \quad \text { for } L \in \mathcal{L}_{k}^{n}
$$

(which depends on the Euclidean structure) and call $\sigma_{k, B}$ the scaling function; then

$$
\alpha_{k, B}(M)=\sigma_{k, B}\left(L_{M}\right) \lambda_{k}(M) .
$$

A 1-normalizing function is uniquely determined, hence $\sigma_{1, B}=1$, thus for $k=1$ we get the Minkowskian curve length vol $_{1}$ again.

Now we can study the existence of Crofton formulae. We assume that a $k$ normalizing function $\alpha_{k}$ and a Minkowski space $\left(\mathbb{R}^{n},\|\cdot\|\right)$ with unit ball $B$ are given.

Definition. A Crofton measure for $\alpha_{k, B}$ is a translation invariant signed measure $\eta_{n-k}$ on $\mathcal{E}_{n-k}^{n}$ for which

$$
\int_{\mathcal{E}_{n-k}^{n}} \operatorname{card}(M \cap E) \eta_{n-k}(\mathrm{~d} E)=c \alpha_{k, B}(M)
$$


holds for every $k$-dimensional convex body $M$, with a positive constant $c$ (which will be set equal to 1 in the following).

It is no essential restriction of the generality to consider here only convex $k$-dimensional sets $M$. If (73) holds for these, the formula can be extended to more general $k$-dimensional surfaces.

Let us suppose that a Crofton measure $\eta_{n-k}$ for $\alpha_{k, B}$ exists. Since it is translation invariant and locally finite, there is a finite signed measure $\varphi$ on $\mathcal{L}_{n-k}^{n}$ such that

$$
\int_{\mathcal{E}_{n-k}^{n}} f \mathrm{~d} \eta_{n-k}=\int_{\mathcal{L}_{n-k}^{n}} \int_{L^{\perp}} f(L+x) \lambda_{k}(\mathrm{~d} x) \varphi(\mathrm{d} L)
$$

holds for every nonnegative measurable function $f$ on $\mathcal{E}_{n-k}^{n}$ (see, e.g., [52, 4.1.1]). Let $M$ be a $k$-dimensional convex body. For a subspace $L$, we denote by $\mid L$ the orthogonal projection to $L$. With the function $[\cdot, \cdot]$ introduced in Subsection 2.1, we have $\lambda_{k}\left(M \mid L^{\perp}\right)=\lambda_{k}(M)\left[L_{M}, L\right]$. Now we obtain

$$
\begin{aligned}
\alpha_{k, B}(M) & =\int_{\mathcal{E}_{n-k}^{n}} \operatorname{card}(M \cap E) \eta_{n-k}(\mathrm{~d} E) \\
& =\int_{\mathcal{E}_{n-k}^{n}} \mathbf{1}\{M \cap E \neq \emptyset\} \eta_{n-k}(\mathrm{~d} E) \\
& =\int_{\mathcal{L}_{n-k}^{n}} \int_{L^{\perp}} \mathbf{1}\{M \cap(L+x) \neq \emptyset\} \lambda_{k}(\mathrm{~d} x) \varphi(\mathrm{d} L) \\
& =\int_{\mathcal{L}_{n-k}^{n}} \lambda_{k}\left(M \mid L^{\perp}\right) \varphi(\mathrm{d} L) \\
& =\lambda_{k}(M) \int_{\mathcal{L}_{n-k}^{n}}\left[L_{M}, L\right] \varphi(\mathrm{d} L) .
\end{aligned}
$$

This yields

$$
\sigma_{k, B}(E)=\int_{\mathcal{L}_{n-k}^{n}}[E, L] \varphi(\mathrm{d} L) \quad \text { for } E \in \mathcal{L}_{k}^{n} .
$$

Conversely, if (75) is satisfied with a finite signed measure $\varphi$, then we can use (74) to define a signed measure $\eta_{n-k}$ on $\mathcal{E}_{n-k}^{n}$, and this is a Crofton measure for $\alpha_{k, B}$.

The crucial integral equation (75) is now first considered for $k=1$. Choosing $v \in S^{n-1}$ and for $M$ the segment with endpoints $v$ and $-v$, for which $\alpha_{1, B}(M)=\operatorname{vol}_{1}(M)=2\|v\|$, and representing $(n-1)$-dimensional linear subspaces by their Euclidean unit normal vectors, we see that (75) is equivalent to

$$
\|v\|=\int_{S^{n-1}}|\langle u, v\rangle| \rho(\mathrm{d} u)
$$

with an even finite signed measure $\rho$ on $S^{n-1}$. 
Introducing the polar unit ball (the dual body of $B$, where $\mathbb{R}^{n}$ and its dual space have been identified via the scalar product),

$$
B^{o}:=\left\{x \in \mathbb{R}^{n}:\langle x, y\rangle \leq 1 \text { for all } y \in B\right\},
$$

we have $\|v\|=h\left(B^{o}, v\right)$ (e.g., [39, p. 44]); hence (75), for $k=1$, is equivalent to

$$
h\left(B^{o}, v\right)=\int_{S^{n-1}}|\langle u, v\rangle| \rho(\mathrm{d} u) \quad \text { for } v \in \mathbb{R}^{n} .
$$

A convex body $B^{o}$ whose support function has a representation (76) with a finite signed measure $\rho$ is called a generalized zonoid, and it is a zonoid (as defined in Subsection 1.3, i.e., a convex body which can be approximated by finite vector sums of line segments) if there is such a representation with a positive measure $\rho$. Every body in $\mathcal{C}^{n}$ with sufficiently smooth support function is a generalized zonoid. Therefore, the generalized zonoids are dense in the space $\mathcal{C}^{n}$, whereas the zonoids are nowhere dense in $\mathcal{C}^{n}$. The crosspolytope is an example of a centrally symmetric convex body which is not a generalized zonoid. Hence, in the Minkowski space $\ell_{\infty}^{n}$, no Crofton measure for vol ${ }_{1}$ exists.

Now we suppose that a positive Crofton measure for vol $_{1}$ exists, and we draw a second conclusion. For this, we choose $m$ points $p_{1}, \ldots, p_{m} \in \mathbb{R}^{n}$ and integers $N_{1}, \ldots, N_{m}$ with

$$
\sum_{i=1}^{m} N_{i}=1
$$

Let $H$ be a hyperplane not incident with one of the points $p_{1}, \ldots, p_{m}$, and let $H^{+}, H^{-}$be the two closed halfspaces bounded by $H$. Then, denoting the segment with endpoints $p_{i}, p_{j}$ by $\overline{p_{i} p_{j}}$, we have

$$
\begin{aligned}
\sum_{i<j} 1\left\{H \cap \overline{p_{i} p_{j}} \neq \emptyset\right\} N_{i} N_{j} & =\left(\sum_{p_{i} \in H^{+}} N_{i}\right)\left(\sum_{p_{j} \in H^{-}} N_{j}\right) \\
& =\left(\sum_{p_{i} \in H^{+}} N_{i}\right)\left(1-\sum_{p_{i} \in H^{+}} N_{i}\right) \\
& \leq 0,
\end{aligned}
$$

where we have used $(77)$ and the fact that $z(1-z) \leq 0$ for every integer $z$. Integrating the obtained inequality over all $H \in \mathcal{E}_{n-1}^{n}$ with respect to the positive measure $\eta_{n-1}$ (and observing that the set of hyperplanes through one of the points $p_{1}, \ldots, p_{m}$ has measure zero), we obtain

$$
\sum_{i<j} d\left(p_{i}, p_{j}\right) N_{i} N_{j} \leq 0 .
$$

Generally, a metric $d$ satisfying (78) for all $m$-tuples $\left(N_{1}, \ldots, N_{m}\right)$ of integers with (77) and all $m \in \mathbb{N}$ is called a hypermetric. We say that our Minkowski 
space $\left(\mathbb{R}^{n},\|\cdot\|\right)$ is hypermetric if its induced metric is a hypermetric. Now we can formulate a theorem.

Theorem 4.1. In the Minkowski space $\left(\mathbb{R}^{n},\|\cdot\|\right)$ with unit ball B, a Crofton measure for $\mathrm{vol}_{1}$ exists if and only if the polar unit ball $B^{\circ}$ is a generalized zonoid. The following conditions are equivalent:

(a) There exists a positive Crofton measure for vol $_{1}$.

(b) The polar unit ball $B^{\circ}$ is a zonoid.

(c) The Minkowski space $\left(\mathbb{R}^{n},\|\cdot\|\right)$ is hypermetric.

For the implication $(\mathrm{c}) \Rightarrow(\mathrm{b})$, which we do not prove here, we refer to [1] and the references given there.

We turn to $k$-areas for $k>1$ and first introduce two special cases of such areas, which play a prominent role. The Busemann $k$-area, denoted by $\beta_{k}$, is defined by the constant $k$-normalizing function, $\alpha_{k}(C)=\kappa_{k}$ for $C \in \mathcal{C}^{k}$. The Holmes-Thompson $k$-area is defined by the $k$-normalizing function $\alpha_{k}(C):=\kappa_{k}^{-1} \operatorname{vp}(C)$ for $C \in \mathcal{C}^{k}$, where

$$
\operatorname{vp}(C):=\lambda_{k}(C) \lambda_{k}\left(C^{o}\right)
$$

is the volume product; here the polar body $C^{o}$ is taken with respect to the $k$-dimensional linear subspace containing $C$. The volume product is invariant under linear transformations and therefore independent of the Euclidean structure. The scaling function of the Busemann $k$-area is given by

$$
\sigma_{k, B}^{\mathrm{Bus}}(L)=\frac{\kappa_{k}}{\lambda_{k}(B \cap L)} \quad \text { for } L \in \mathcal{L}_{k}^{n},
$$

and hence the Busemann $k$-area of a $k$-dimensional $C^{1}$ submanifold $M$ by

$$
\beta_{k}(M)=\int_{M} \frac{\kappa_{k}}{\lambda_{k}\left(B \cap T_{x} M\right)} \lambda_{k}(\mathrm{~d} x) .
$$

For the scaling function of the Holmes-Thompson $k$-area we obtain $\sigma_{k, B}^{\mathrm{HT}}(L)=$ $\kappa^{-1} \lambda_{k}\left((B \cap L)^{o}\right)$. Convex geometry tells us that $(B \cap L)^{o}=B^{o} \mid L$, hence

$$
\sigma_{k, B}^{\mathrm{HT}}(L)=\frac{\lambda_{k}\left(B^{o} \mid L\right)}{\kappa_{k}} \quad \text { for } L \in \mathcal{L}_{k}^{n} .
$$

We denote the Holmes-Thompson $k$-area by $\operatorname{vol}_{k}$; then

$$
\operatorname{vol}_{k}(M)=\int_{M} \frac{\lambda_{k}\left(B^{o} \mid T_{x} M\right)}{\kappa_{k}} \lambda_{k}(\mathrm{~d} x)
$$

for a $k$-dimensional $C^{1}$ submanifold $M$. 
Let $\mathcal{H}_{B}^{k}$ denote the $k$-dimensional Hausdorff measure that is induced by the metric $d$ of our Minkowski space. It can be shown that the Busemann $k$-area $\beta_{k}(M)$ is nothing but $\mathcal{H}_{B}^{k}(M)$ (if the Hausdorff measure is suitably normalized). Using this Hausdorff measure instead of the Euclidean area measure $\lambda_{k}$, we can write the Holmes-Thompson area in the form

$$
\operatorname{vol}_{k}(M)=\frac{1}{\kappa_{k}^{2}} \int_{M} \operatorname{vp}\left(B \cap T_{x} M\right) \mathcal{H}_{B}^{k}(\mathrm{~d} x),
$$

which does not use the auxiliary Euclidean structure any more.

Now we study the existence of Crofton measures for a general Minkowskian $(n-1)$-area $\alpha_{n-1, B}$. Writing

$$
\sigma(u):=\langle u, u\rangle^{1 / 2} \sigma_{n-1, B}\left(u^{\perp}\right) \quad \text { for } u \in \mathbb{R}^{n} \backslash\{0\},
$$

we see that (75) for $k=n-1$ is equivalent to

$$
\sigma(u)=\int_{S^{n-1}}|\langle u, v\rangle| \rho(\mathrm{d} v), \quad u \in S^{n-1},
$$

with an even finite signed measure $\rho$ on $S^{n-1}$.

Suppose that a positive Crofton measure exists for $\alpha_{n-1, B}$. Then $\rho$ in (83) is a positive measure, hence $\sigma$ is the support function of a zonoid. This zonoid is denoted by $\mathbf{I}_{\alpha, B}$ and called the isoperimetrix. The name comes from the isoperimetric problem: it can be shown that a convex body in $\mathbb{R}^{n}$ with given positive volume has smallest $(n-1)$-area $\alpha_{n-1, B}$ of its boundary if and only if it is homothetic to the isoperimetrix.

Conversely, if (83) holds with a positive measure $\rho$, then there exists a positive Crofton measure for the $(n-1)$-area $\alpha_{n-1, B}$. For the Holmes-Thompson $(n-1)$-area this is always the case, in any Minkowski space. In fact, from a well-known formula for projection volumes of convex bodies, we get for the Holmes-Thompson area

$$
\sigma(u)=\frac{1}{\kappa_{n-1}} \lambda_{n-1}\left(B^{o} \mid u^{\perp}\right)=\frac{1}{2 \kappa_{n-1}} \int_{S^{n-1}}|\langle u, v\rangle| S_{n-1}\left(B^{o}, \mathrm{~d} v\right)
$$

for $u \in S^{n-1}$, and here the area measure $S_{n-1}\left(B^{o}, \cdot\right)$ is positive.

For the Busemann $(n-1)$-area, it can be shown that the function $\sigma$ is again a support function, but not necessarily of a zonoid or a generalized zonoid. As a consequence, there need not exist a Crofton measure for the Busemann $(n-1)$-area. More precise information is contained in the following theorem, whose proof is based on properties of zonoids and generalized zonoids. Here, $C^{n}$ denotes a cube in $\mathbb{R}^{n}$ with centre 0 .

Theorem 4.2. (a) There exist Minkowski spaces, with unit ball arbitrarily close to $B^{n}$, in which there is no Crofton measure for the Busemann $(n-1)$ area. There also exist Minkowski spaces with unit ball arbitrarily close to $B^{n}$, 
but different from an ellipsoid, in which there is a positive Crofton measure for the Busemann $(n-1)$-area.

(b) In every Minkowski space of sufficiently large dimension $n$ and with unit ball sufficiently close to $C^{n}$, there is no positive Crofton measure for the Busemann $(n-1)$-area.

(c) There exist Minkowski spaces, for example $\ell_{\infty}^{n}$ and $\ell_{1}^{n}$, in which there is no positive Crofton measure for any general Minkowskian (n-1)-area, except for the multiples of the Holmes-Thompson $(n-1)$-area.

(d) In every Minkowski space, there is a positive Crofton measure for the Holmes-Thompson $(n-1)$-area.

The preceding theorem is sufficient reason for us to concentrate, from now on, on the Holmes-Thompson area. This is even more justified in view of the following theorem.

Theorem 4.3. Let $\left(\mathbb{R}^{n},\|\cdot\|\right)$ be a Minkowski space. If in this space there exists a Crofton measure (a positive Crofton measure) for vol $_{1}$, then there also exists a Crofton measure (a positive Crofton measure) for $\operatorname{vol}_{k}, k \in\{2, \ldots, n-2\}$.

Proof. Suppose that in the Minkowski space with unit ball $B$ there is a Crofton measure for $\operatorname{vol}_{1}$. Then, by (76), there is an even finite signed measure $\rho$ on $S^{n-1}$ such that

$$
h\left(B^{o}, u\right)=\int_{S^{n-1}}|\langle u, v\rangle| \rho(\mathrm{d} v) \quad \text { for } u \in \mathbb{R}^{n} .
$$

We employ a result from the theory of generalized zonoids. For vectors $u_{1}, \ldots, u_{k} \in S^{n-1}$, we denote by $L\left(u_{1}, \ldots, u_{k}\right)$ the linear subspace spanned by these vectors and by $\left[u_{1}, \ldots, u_{k}\right]$ the $k$-dimensional Euclidean volume of the parallelepiped spanned by them. Then for $E \in \mathcal{L}_{k}^{n}$ we have

$$
\begin{aligned}
& \lambda_{k}\left(B^{o} \mid E\right) \\
& =\frac{2^{k}}{k !} \int_{S^{n-1}} \cdots \int_{S^{n-1}}\left[E, L\left(u_{1}, \ldots, u_{k}\right)^{\perp}\right]\left[u_{1}, \ldots, u_{k}\right] \rho\left(\mathrm{d} u_{1}\right) \cdots \rho\left(\mathrm{d} u_{k}\right)
\end{aligned}
$$

(see [56], and observe that the proof is valid if $\rho$ is a signed measure). We define a signed measure $\rho^{(k)}$ on $\mathcal{L}_{k}^{n}$ by

$$
\rho^{(k)}(A):=c_{k} \int_{S^{n-1}} \ldots \int_{S^{n-1}} \mathbf{1}_{A}\left(L\left(u_{1}, \ldots, u_{k}\right)\right)\left[u_{1}, \ldots, u_{k}\right] \rho\left(\mathrm{d} u_{1}\right) \cdots \rho\left(\mathrm{d} u_{k}\right)
$$

for Borel sets $A \subset \mathcal{L}_{k}^{n}$, where

$$
c_{k}=\frac{2^{k}}{k ! \kappa_{k}}
$$


Then we can write $(80)$ in the form

$$
\sigma_{k, B}^{H T}(E)=\int_{\mathcal{L}_{k}^{n}}\left[E, L^{\perp}\right] \rho^{(k)}(\mathrm{d} L)=\int_{\mathcal{L}_{n-k}^{n}}[E, L] \rho_{(k)}(\mathrm{d} L)
$$

for $E \in \mathcal{L}_{k}^{n}$; here $\rho_{(k)}$ is the image measure of $\rho^{(k)}$ under the map $L \mapsto L^{\perp}$ from $\mathcal{L}_{k}^{n}$ to $\mathcal{L}_{n-k}^{n}$. We see that the integral equation (75) has a solution for $\sigma_{k, B}^{H T}$, hence there is a Crofton measure for $\operatorname{vol}_{k}$.

If there is a positive Crofton measure for $\operatorname{vol}_{1}$, then $\rho$ is a positive measure, which implies that $\rho_{(k)}$ is a positive measure, hence there is a positive Crofton measure for $\operatorname{vol}_{k}$.

In the proof of Theorem 4.3 we started with a Crofton measure for vol ${ }_{1}$, say $\eta$, and we constructed a Crofton measure for $\operatorname{vol}_{k}$, say $\eta_{n-k}$. This construction has a nice geometric interpretation. Given is a measure $\eta$ on the space of hyperplanes, and we need a measure on the space of $(n-k)$-planes. It turns out that $\eta_{n-k}$, as constructed, is the image measure of $c_{k} \eta^{\otimes k}\left\llcorner\mathcal{H}_{k}^{*}\right.$ under the intersection map

$$
\left(H_{1}, \ldots, H_{k}\right) \mapsto H_{1} \cap \cdots \cap H_{k}
$$

from $\mathcal{H}_{k}^{*}$ to $\mathcal{E}_{n-k}^{n}$, where $\mathcal{H}_{k}^{*}$ denotes the set of all $k$-tuples $\left(H_{1}, \ldots, H_{k}\right)$ of hyperplanes with $\operatorname{dim}\left(H_{1} \cap \cdots \cap H_{k}\right)=n-k$ and $L$ denotes restriction. More explicitly, for $\mathcal{A} \in \mathcal{B}\left(\mathcal{E}_{n-k}^{n}\right)$ we have

$$
\eta_{n-k}(\mathcal{A})=c_{k} \underbrace{\eta \otimes \cdots \otimes \eta}_{k}\left(\left\{\left(H_{1}, \ldots, H_{k}\right) \in \mathcal{H}_{k}^{*}: H_{1} \cap \cdots \cap H_{k} \in \mathcal{A}\right\}\right) .
$$

There are two main cases where the assumption of Theorem 4.3 is satisfied. If the norm $\|\cdot\|$, which is equal to $h\left(B^{o}, \cdot\right)$, is sufficiently smooth, then $B^{o}$ is a generalized zonoid, hence a Crofton measure exists for vol ${ }_{1}$. If the Minkowski space $\left(\mathbb{R}^{n},\|\cdot\|\right)$ is hypermetric, then Theorem 4.1 says that a positive Crofton measure for vol $_{1}$ exists. Hence, in either of these two cases, the Holmes-Thompson area of any dimension satisfies an intersection formula of Crofton type.

Hints to the literature. Motivated by earlier work of Busemann, a study of integral geometric formulae for areas in affine spaces, and particularly in Minkowski spaces, was made in [53]. Much of the material exposed here, including the proof of Theorem 4.3, is found there, together with additional information. Parts (a) and (b) of Theorem 4.2 are proved in [44], and part (c) in [41].

\subsection{Projective Finsler Spaces}

The main result of the previous subsection, the existence of Crofton measures for Holmes-Thompson areas in smooth or hypermetric Minkowski spaces, can 
be extended to certain spaces where one has no longer any nontrivial invariance under a transformation group. We sketch here, without proofs, the main ideas of such an extension. It takes place in a natural generalization of Minkowski spaces, the projective Finsler spaces. Generally speaking, a Finsler space is a differentiable manifold together with a norm in each tangent space, satisfying certain smoothness assumptions. Here we consider only $\mathbb{R}^{n}$ as the underlying manifold (where we canonically identify each tangent space of $\mathbb{R}^{n}$ with $\mathbb{R}^{n}$ itself), and we consider Finsler metrics on $\mathbb{R}^{n}$ which are compatible with the affine structure of $\mathbb{R}^{n}$, in the sense we shall now make precise.

By a Finsler metric on $\mathbb{R}^{n}$ we understand here a continuous function $F: \mathbb{R}^{n} \times \mathbb{R}^{n} \rightarrow[0, \infty)$ such that $F(x, \cdot)=:\|\cdot\|_{x}$ is a norm on $\mathbb{R}^{n}$ for each $x \in \mathbb{R}^{n}$. If this holds, the pair $\left(\mathbb{R}^{n}, F\right)$ is called a Finsler space. In such a Finsler space, the length of a parameterized piecewise $C^{1}$ curve $\gamma:[a, b] \rightarrow$ $\mathbb{R}^{n}$ is defined by

$$
L_{F}(\gamma):=\int_{a}^{b} F\left(\gamma(t), \gamma^{\prime}(t)\right) \mathrm{d} t ;
$$

this is independent of the parameterization since $F(\gamma(t), \cdot)$ is a norm. For $p, q \in \mathbb{R}^{n}$, the distance $d_{F}(p, q)$ is defined as the infimum of the lengths of all piecewise $C^{1}$ curves connecting $p$ and $q$. Then $d_{F}$ is a metric, called the metric induced by $F$. The Finsler space $\left(\mathbb{R}^{n}, F\right)$ is called projective if line segments are shortest curves connnecting their endpoints. If this holds, the segment with endpoints $p, q$ has length $d_{F}(p, q)$.

Let $\left(\mathbb{R}^{n}, F\right)$ be a Finsler space. For $x \in \mathbb{R}^{n}$, the unit ball of the Minkowski space $\left(\mathbb{R}^{n},\|\cdot\|_{x}\right)$ is denoted by $B_{x}$ (recall that we have identified the tangent space $T_{x} \mathbb{R}^{n}$ of $\mathbb{R}^{n}$ at $x$ with $\mathbb{R}^{n}$ ). As in the previous subsection, we use a fixed auxiliary scalar product $\langle\cdot, \cdot\rangle$ on $\mathbb{R}^{n}$. With its aid, we define the polar body of $B_{x}$

$$
B_{x}^{o}:=\left\{v \in \mathbb{R}^{n}:\langle u, v\rangle \leq 1 \text { for all } u \in B_{x}\right\} .
$$

(Without our simplifying conventions, $B_{x}$ would be a convex body in the tangent space $T_{x} \mathbb{R}^{n}$, and $B_{x}^{o}$ would be a convex body in the dual tangent space $T_{x}^{*} \mathbb{R}^{n}$.)

Extending the definition given for Minkowski spaces, one can define the Holmes-Thompson $k$-area of a $k$-dimensional $C^{1}$-submanifold $M$ in $\left(\mathbb{R}^{n}, F\right)$ by

$$
\operatorname{vol}_{k}(M)=\frac{1}{\kappa_{k}} \int_{M} \lambda_{k}\left(B_{x}^{o} \mid T_{x} M\right) \lambda_{k}(\mathrm{~d} x) .
$$

This definition uses the Euclidean structure in several ways, but is, in fact, independent of its choice. Formula (82), which does not use the Euclidean structure, can be extended as follows. If $\mathcal{H}_{F}^{k}$ denotes the $k$-dimensional Hausdorff measure induced by the metric $d_{F}$, then

$$
\operatorname{vol}_{k}(M)=\frac{1}{\kappa_{k}^{2}} \int_{M} \operatorname{vp}\left(B_{x} \cap T_{x} M\right) \mathcal{H}_{F}^{k}(\mathrm{~d} x) .
$$


As in the special case of Minkowski spaces, the existence of Crofton measures (which we define in the same way) for Holmes-Thompson areas is closely related to the theory of generalized zonoids. First, we study this connection under a smoothness assumption. We assume that the Finsler space $\left(\mathbb{R}^{n}, F\right)$ is smooth, meaning that the function $F$ is of class $C^{\infty}$ (weaker assumptions would be sufficient, but this is not an issue here).

Let $x \in \mathbb{R}^{n}$ be given. Due to the smoothness assumption, the integral equation

$$
F(x, v)=\int_{S^{n-1}}|\langle u, v\rangle| \gamma_{x}(u) \sigma_{n-1}(\mathrm{~d} u)
$$

has a continuous even solution $\gamma_{x}$ on $S^{n-1}$ (see, e.g., [39, Theorem 3.5.3]). Now the assumption that the Finsler space $\left(\mathbb{R}^{n}, F\right)$ is projective has a strong implication on $\gamma_{x}(u)$, as a function of its two variables $x$ and $u$ : there exists a continuous function $g: S^{n-1} \times \mathbb{R} \rightarrow \mathbb{R}$ such that $g(u, \tau)=g(-u,-\tau)$ and

$$
\gamma_{x}(u)=g(u,\langle x, u\rangle) \quad \text { for }(x, u) \in \mathbb{R}^{n} \times \mathbb{R}^{n} .
$$

This follows from Pogorelov's [27] work on Hilbert's Fourth Problem (see [45] for a brief sketch of the main ideas). Recalling that $F(x, \cdot)=\|\cdot\|_{x}=h\left(B_{x}^{o}, \cdot\right)$, which is the support function of the polar unit ball at $x$, we now have

$$
h\left(B_{x}^{o}, v\right)=\int_{S^{n-1}}|\langle u, v\rangle| g(u,\langle x, u\rangle) \sigma_{n-1}(\mathrm{~d} u)
$$

for $v \in \mathbb{R}^{n}$. This representation is of the form of (76) and can be employed in a similar way. We use it for the construction of signed measures $\eta_{n-k}$ on the space $\mathcal{E}_{n-k}^{n}$ of $(n-k)$-flats, as in the proof of Theorem 4.3 and the subsequent remark, in the following way. The function $x \mapsto g(u,\langle x, u\rangle)$ is constant on the hyperplane $H$ through $x$ with normal vector $u$, let $h(H)$ be its value on $H$. This defines a function $h$ on $\mathcal{E}_{n-1}^{n}$. Let $\eta$ be the signed measure on $\mathcal{E}_{n-1}^{n}$ which has density $h$ with respect to the rigid motion invariant measure on $\mathcal{E}_{n-1}^{n}$. Explicitly, this comes down to the following. We parameterize hyperplanes of $\mathbb{R}^{n}$ by

$$
H_{u, t}=\left\{y \in \mathbb{R}^{n}:\langle y, u\rangle=t\right\}, \quad u \in S^{n-1}, t \in \mathbb{R},
$$

and then define a signed measure $\eta$ on $\mathcal{E}_{n-1}^{n}$ by

$$
\eta(\mathcal{A}):=\int_{S^{n-1}} \int_{\mathbb{R}} \mathbf{1}_{\mathcal{A}}\left(H_{u, t}\right) g(u, t) \mathrm{d} t \sigma_{n-1}(\mathrm{~d} u)
$$

for $\mathcal{A} \in \mathcal{B}\left(\mathcal{E}_{n-1}^{n}\right)$. Next, we define $\eta_{n-k}$ as the image measure of $c_{k} \eta^{\otimes k}$ under the intersection map $\left(H_{1}, \ldots, H_{k}\right) \mapsto H_{1} \cap \cdots \cap H_{k}$ (with $c_{k}$ given by (86)). Explicitly, this means that

$$
\begin{aligned}
\eta_{n-k}(\mathcal{A}):= & c_{k} \int_{S^{n-1}} \cdots \int_{S^{n-1}} \int_{\mathbb{R}} \ldots \int_{\mathbb{R}} \mathbf{1}_{\mathcal{A}}\left(H_{u_{1}, t_{1}} \cap \cdots \cap H_{u_{k}, t_{k}}\right) \\
& g\left(u_{1}, t_{1}\right) \cdots g\left(u_{k}, t_{k}\right) \mathrm{d} t_{1} \cdots \mathrm{d} t_{k} \sigma_{n-1}\left(\mathrm{~d} u_{1}\right) \cdots \sigma_{n-1}\left(\mathrm{~d} u_{k}\right)
\end{aligned}
$$


for $\mathcal{A} \in \mathcal{B}\left(\mathcal{E}_{n-k}^{n}\right)$.

With these measures, we can now state Crofton formulae in a very general version. A set $M \subset \mathbb{R}^{n}$ is called $\left(\mathcal{H}^{k}, k\right)$-rectifiable, for $k \in\{1, \ldots, n\}$, if $\mathcal{H}^{k}(M)<\infty$ and there are Lipschitz maps $f_{i}: \mathbb{R}^{k} \rightarrow \mathbb{R}^{n}(i \in \mathbb{N})$ such that $\mathcal{H}^{k}\left(M \backslash \bigcup_{i \in \mathbb{N}} f_{i}\left(\mathbb{R}^{k}\right)\right)=0$. Here the $k$-dimensional Hausdorff measure $\mathcal{H}^{k}$ and the notion of Lipschitz map refer to a Euclidean structure, but the class of $\left(\mathcal{H}^{k}, k\right)$-rectifiable sets is independent of the choice of this structure. The definition of the Holmes-Thompson $k$-area can be extended to $\left(\mathcal{H}^{k}, k\right)$ rectifiable Borel sets, for example by (87).

Theorem 4.4. Let $\left(\mathbb{R}^{n}, F\right)$ be a smooth projective Finsler space. Then, for $k \in\{1, \ldots, n-1\}$ and every $\left(\mathcal{H}^{k}, k\right)$-rectifiable Borel set $M$ in $\mathbb{R}^{n}$,

$$
\int_{\mathcal{E}_{n-k}^{n}} \operatorname{card}(M \cap E) \eta_{n-k}(\mathrm{~d} E)=\operatorname{vol}_{k}(M) .
$$

An even more general version holds. This refers to the case where $M$ and the intersecting flats are not necessarily of complementary dimensions. For $j \in\{1, \ldots, n-1\}$ and $k \in\{n-j, \ldots, n\}$, and for $\left(\mathcal{H}^{k}, k\right)$-rectifiable Borel sets $M$,

$$
\int_{\mathcal{E}_{j}^{n}} \operatorname{vol}_{k+j-n}(M \cap E) \eta_{j}(\mathrm{~d} E)=\frac{c_{k+j-n} c_{n-j}}{c_{k}} \operatorname{vol}_{k}(M) .
$$

We turn to the existence of positive Crofton measures. A projective Finsler space $\left(\mathbb{R}^{n}, F\right)$ is called hypermetric if its induced metric $d_{F}$ is a hypermetric. We assume now that the Finsler space $\left(\mathbb{R}^{n}, F\right)$ is smooth, projective, and hypermetric. Then every polar local unit ball $B_{x}^{o}$ is not only a generalized zonoid (which it is by (88)), but even a zonoid (as proved in [1]), which means that the function $g(\cdot,\langle x, \cdot\rangle)$ in (88), and hence $g$, is nonnegative. It follows that each $\eta_{n-k}$ is a positive measure.

The existence of positive Crofton measures, as just established, can be extended to general (i.e., not necessarily smooth) hypermetric projective Finsler spaces. Such an extension can be based on the following approximation result, which Pogorelov [27] and Szabó [54] established in their work on Hilbert's Fourth Problem. For every $\epsilon>0$, there is a smooth projective Finsler space $\left(\mathbb{R}^{n}, F_{\epsilon}\right)$ such that $\lim _{\epsilon \rightarrow 0} F_{\epsilon}=F$, uniformly on every compact set. Moreover, each $\left(\mathbb{R}^{n}, F_{\epsilon}\right)$ is hypermetric (see [1]). Therefore, to every $\epsilon$, there exists a positive Crofton measure $\eta_{n-1}$ for the Holmes-Thompson 1-area, as constructed above. This measure depends on $\epsilon$; we denote it by $\eta_{(\epsilon)}$. Making essential use of the positivity of these measures, one can show that the family $\left(\eta_{(\epsilon)}\right)_{\epsilon \in(0,1)}$ of measures is relatively compact in the vague topology. Hence, there is a sequence $\left(\epsilon_{i}\right)_{i \in \mathbb{N}}$ tending to zero such that the sequence $\left(\eta_{\left(\epsilon_{i}\right)}\right)_{i \in \mathbb{N}}$ converges vaguely to a measure $\eta$ on $\mathcal{E}_{n-1}^{n}$. With this measure, we repeat the earlier construction: we define the measure $\eta_{n-k}$ on $\mathcal{E}_{n-k}^{n}$ as the image measure of 
$c_{k} \eta^{\otimes k}\left\llcorner\mathcal{H}_{k}^{*}\right.$ under the map $\left(H_{1}, \ldots, H_{k}\right) \mapsto H_{1} \cap \cdots \cap H_{k}$ from $\mathcal{H}_{k}^{*}$ to $\mathcal{E}_{n-k}^{n}$. Using the vague convergence of $\left(\eta_{\left(\epsilon_{i}\right)}\right)_{i \in \mathbb{N}}$ to $\eta$, it can be proved that

$$
\int_{\mathcal{E}_{n-k}^{n}} \operatorname{card}(M \cap E) \eta_{n-k}(\mathrm{~d} E)=\operatorname{vol}_{k}(M)
$$

for $k \in\{1, \ldots, n-1\}$ and every $k$-dimensional convex body $M$. By definition, $\eta_{n-k}$ is a positive Crofton measure for the Holmes-Thompson $k$-area in $\left(\mathbb{R}^{n}, F\right)$. However, it has not been investigated, in this case of a general Finsler metric, whether $(93)$ can be extended to $\left(\mathcal{H}^{k}, k\right)$-rectifiable Borel sets $M$, nor whether (92) has a counterpart.

We remark that a measure $\eta_{n-k}$ satisfying (93) for all $k$-dimensional convex bodies $M$ is uniquely determined if either $k=1$ or $k=n-1$, but not in the intermediate cases.

Theorem 4.2(d) can be extended from Minkowski spaces to projective Finsler spaces, thus a positive Crofton measure for the Holmes-Thompson $(n-1)$-area in the projective Finsler space $\left(\mathbb{R}^{n}, F\right)$ exists even if the space is not hypermetric. The clue for a proof of this fact is again a formula from the theory of generalized zonoids. First we assume that $\left(\mathbb{R}^{n}, F\right)$ is a smooth projective Finsler space. Let $s_{n-1}\left(B_{x}^{o}, u\right)$ denote the product of the principal radii of curvature of the boundary of $B_{x}^{o}$ at the point with outer normal vector $u \in S^{n-1}$. From the representation (88), one has an explicit integral representation of $s_{n-1}\left(B_{x}^{o}, u\right)$ in terms of the function $g$; see [55, Satz 7]. With its aid, one can show that a function $\delta$ on $\mathcal{E}_{1}^{n}$ can be defined by

$$
\delta(\operatorname{lin}\{u\}+x):=s_{n-1}\left(B_{x}^{o}, u\right) \quad \text { for } u \in S^{n-1}, x \in \mathbb{R}^{n}
$$

and that this function is a density of the signed measure $\eta_{1}$ with respect to the suitably normalized rigid motion invariant measure on the space $\mathcal{E}_{1}^{n}$ of lines. Since this density is always nonnegative, the Crofton measure $\eta_{1}$ is positive. The existence of a positive Crofton measure for the Holmes-Thompson $(n-1)$ area in non-smooth projective Finsler spaces can then again be obtained by approximation.

We collect the stated results in a theorem.

Theorem 4.5. Let $\left(\mathbb{R}^{n}, F\right)$ be a projective Finsler space. In this space, there exists a positive Crofton measure for the Holmes-Thompson (n-1)-area. If the space is hypermetric, there exists a positive Crofton measure for the HolmesThompson $k$-area, for each $k \in\{1, \ldots, n-1\}$.

Hints to the literature. For smooth projective Finsler spaces and smooth submanifolds, a version of the Crofton formula (91) was proved in [2], using the symplectic structure on the space of geodesics of a projective Finsler space. The general formula (92), together with its proof based on the theory of generalized zonoids, appears in [46]. Theorem 4.5 was proved in [45]. 


\subsection{Nonstationary hyperplane processes}

Finally, we treat a special topic from stochastic geometry which is closely related to the preceding subsection. The relation to Crofton measures in projective Finsler spaces is not one of application, but consists in the similarity of the underlying structures. We study stochastic processes of $k$-planes in $\mathbb{R}^{n}$, and in particular of hyperplanes.

First we need some explanations. Let $S$ be an arbitrary locally compact space with a countable base. A subset $F \subset S$ is called locally finite if $F \cap C$ is finite for every compact subset $C$ of $S$. Let $\mathcal{F}_{l f}$ be the system of all locally finite subsets of $S$. One equips $\mathcal{F}_{l f}$ with the smallest $\sigma$-algebra for which all counting functions

$$
F \mapsto \operatorname{card}(F \cap A), \quad A \in \mathcal{B}(S),
$$

are measurable. A simple point process in $S$ is a random variable $X$ on some probability space $(\Omega, \mathrm{A}, \mathbb{P})$ with values in $\mathcal{F}_{l f}$. The expectation

$$
\Theta(A):=\mathbb{E} \operatorname{card}(X \cap A), \quad A \in \mathcal{B}(S),
$$

defines the intensity measure of the point process $X$. The point process $X$ with intensity measure $\Theta$ is called a Poisson process if $\Theta$ is finite on compact sets and if, for every Borel set $A \subset S$ with $\Theta(A)<\infty$ and all $j \in \mathbb{N}_{0}$ one has

$$
\mathbb{P}(\operatorname{card}(X \cap A)=j)=\frac{\Theta(A)^{j}}{j !} e^{-\Theta(A)} .
$$

This is now applied to the space $S=\mathcal{E}_{k}^{n}$, where $k \in\{0, \ldots, n-1\}$. We assume that $X$ is a simple point process in $\mathcal{E}_{k}^{n}$, with an intensity measure $\Theta \not \equiv 0$ which is finite on compact sets. We call $X$ a $k$-flat process. The process $X$ is stationary (isotropic) if its distribution is invariant under translations (rotations). Stationary $k$-flat processes have been thoroughly studied in stochastic geometry. We work here with a weaker assumption.

Definition. The $k$-flat process $X$ is regular if its intensity measure has a continuous density with respect to some translation-invariant, locally finite measure on $\mathcal{E}_{k}^{n}$.

Let $X$ be a regular $k$-flat process, with intensity measure $\Theta$. Thus, there exist a locally finite, translation invariant measure $\mu$ on $\mathcal{E}_{k}^{n}$ and a nonnegative, continuous function $h$ on $\mathcal{E}_{k}^{n}$ such that

$$
\Theta(\mathcal{A})=\int_{\mathcal{A}} h \mathrm{~d} \mu \quad \text { for } \mathcal{A} \in \mathcal{B}\left(\mathcal{E}_{k}^{n}\right) .
$$

By the decomposition result (74), there is a finite measure $\Phi$ on $\mathcal{L}_{k}^{n}$ such that

$$
\int_{\mathcal{E}_{k}^{n}} f \mathrm{~d} \mu=\int_{\mathcal{L}_{k}^{n}} \int_{L^{\perp}} f(L+x) \lambda_{k}(\mathrm{~d} x) \Phi(\mathrm{d} L)
$$


for every nonnegative, measurable function $f$ on $\mathcal{E}_{k}^{n}$. This gives

$$
\Theta(\mathcal{A})=\int_{\mathcal{L}_{k}^{n}} \int_{L^{\perp}} \mathbf{1}_{\mathcal{A}}(L+x) h(L+x) \lambda_{k}(\mathrm{~d} x) \Phi(\mathrm{d} L)
$$

for $\mathcal{A} \in \mathcal{B}\left(\mathcal{E}_{k}^{n}\right)$.

To measure the local 'denseness' of the process $X$, we consider the expectations

$$
\mathbb{E} \sum_{E \in X} \lambda_{k}(E \cap B)
$$

for $B \in \mathcal{B}\left(\mathbb{R}^{n}\right)$ with $\lambda_{n}(B)<\infty$. One obtains

$$
\mathbb{E} \sum_{E \in X} \lambda_{k}(E \cap B)=\int_{B} \int_{\mathcal{L}_{k}^{n}} h(L+x) \Phi(\mathrm{d} L) \lambda_{n}(\mathrm{~d} x) .
$$

Thus, the measure $\mathbb{E} \sum_{E \in X} \lambda_{k}(E \cap \cdot)$ has a continuous density with respect to Lebesgue measure $\lambda_{n}$, given by

$$
\gamma(x):=\int_{\mathcal{L}_{k}^{n}} h(L+x) \Phi(\mathrm{d} L) .
$$

This function $\gamma$ is called the intensity function of the process $X$. If $X$ is stationary, this function is a constant, called the intensity of $X$. The following more intuitive interpretation of the intensity function can be proved:

$$
\gamma(x)=\lim _{r \rightarrow 0} \frac{1}{\kappa_{n-k} r^{n-k}} \mathbb{E} \operatorname{card}\left(\left\{E \in X: E \cap\left(r B^{n}+x\right) \neq \emptyset\right\}\right) .
$$

From now on we assume that $k=n-1$, so that $X$ is a regular hyperplane process. The density $h$ is now defined on the space $\mathcal{E}_{n-1}^{n}$ of hyperplanes, hence we can define a function $g: S^{n-1} \times \mathbb{R} \rightarrow[0, \infty)$ by

$$
g(u, t):=h\left(H_{u, t}\right) .
$$

Then $g$ is continuous and satisfies $g(u, t)=g(-u,-t)$. The measure $\Phi$ on $\mathcal{L}_{n-1}^{n}$ defines an even measure $\tilde{\Phi}$ on the sphere $S^{n-1}$ satisfying

$$
\tilde{\Phi}(A)=\frac{1}{2} \Phi\left(\left\{H_{u, 0}: u \in A\right\}\right)
$$

for Borel sets $A \subset S^{n-1}$ without antipodal points. Now (94) can be written in the form

$$
\Theta(\mathcal{A})=\int_{S^{n-1}} \int_{\mathbb{R}} \mathbf{1}_{\mathcal{A}}\left(H_{u, t}\right) g(u, t) \mathrm{d} t \tilde{\Phi}(\mathrm{d} u)
$$

for $\mathcal{A} \in \mathcal{B}\left(\mathcal{E}_{n-1}^{n}\right)$. Note that this representation is of the type (89), but is more general. In the preceding subsection, the function $g$ was derived from the local unit balls of a given projective Finsler metric. We will now, reversely, use the 
present function $g$ to construct 'local unit balls' and exhibit their relevance for the geometry of the hyperplane process $X$.

For each $x \in \mathbb{R}^{n}$, we define a finite even measure $\rho_{x}$ on $S^{n-1}$ by

$$
\rho_{x}(A):=\int_{A} g(u,\langle u, x\rangle) \tilde{\Phi}(\mathrm{d} u), \quad A \in \mathcal{B}\left(S^{n-1}\right) .
$$

Then we define the local associated zonoid $\Pi_{x}$ of $X$ at $x$ as the convex body with support function

$$
h\left(\Pi_{x}, u\right)=\int_{S^{n-1}}|\langle u, v\rangle| \rho_{x}(\mathrm{~d} v), \quad u \in \mathbb{R}^{n} .
$$

The main results of this subsection are two examples showing how these local associated zonoids enter the discussion of natural geometric questions about the hyperplane process $X$.

First, let $M \subset \mathbb{R}^{n}$ be a closed line segment. We ask for the expected number of intersection points of $M$ with the hyperplanes of $X$. Putting

$$
\mathcal{H}_{M}:=\left\{H \in \mathcal{E}_{n-1}^{n}: H \cap M \neq \emptyset\right\},
$$

this expected number is given by

$$
\mathbb{E} \operatorname{card}\left(X \cap \mathcal{H}_{M}\right)=\Theta\left(\mathcal{H}_{M}\right)=\int_{S^{n-1}} \int_{\mathbb{R}} \mathbf{1}_{\mathcal{H}_{M}}\left(H_{u, t}\right) g(u, t) \mathrm{d} t \tilde{\Phi}(\mathrm{d} u) .
$$

For the computation of the inner integral, we choose a $C^{1}$ parameterization $y:[a, b] \rightarrow \mathbb{R}^{n}$ of $M$ with $y^{\prime} \neq 0$. Let $u \in S^{n-1}$ be given, without loss of generality not orthogonal to $M$, and let $\mathbf{1}_{\mathcal{H}_{M}}\left(H_{u, t}\right)=1$. Then there is a unique $s \in[a, b]$ with $M \cap H_{u, t}=\{y(s)\}$, hence $t=\langle u, y(s)\rangle$. Substituting $t$ by $s \cdot \operatorname{sgn}\left\langle u, y^{\prime}\right\rangle$, we get

$$
\begin{aligned}
\mathbb{E} \operatorname{card}\left(X \cap \mathcal{H}_{M}\right) & =\int_{S^{n-1}} \int_{a}^{b} g(u,\langle u, y(s)\rangle)\left|\left\langle u, y^{\prime}(s)\right\rangle\right| \mathrm{d} s \tilde{\Phi}(\mathrm{d} u) \\
& =\int_{a}^{b} h\left(\Pi_{y(s)}, y^{\prime}(s)\right) \mathrm{d} s .
\end{aligned}
$$

Defining a Finsler metric $F$ by

$$
F(x, u):=h\left(\Pi_{x}, u\right),
$$

we see that

$$
\mathbb{E} \operatorname{card}\left(X \cap \mathcal{H}_{M}\right)=\int_{a}^{b} F\left(y(s), y^{\prime}(s)\right) \mathrm{d} s,
$$

hence we can formulate the following result. 
Theorem 4.6. The expected number of hyperplanes in the regular hyperplane process $X$ hitting the segment $M$ is equal to the Finsler length of $M$, for the Finsler metric defined by the support function of the local associated zonoids.

For our second question we assume now, in addition, that $X$ is a Poisson process. The question concerns the processes of lower dimensional flats that are generated by intersecting hyperplanes of $X$. For $k \in\{2, \ldots, n\}$ we take, in every realization of $X$, all intersections of any $k$ hyperplanes in $X$ which have linearly independent normal vectors. This defines a simple process of $(n-k)$-flats. We denote it by $X_{k}$, and its intensity measure by $\Theta_{k}$. Using the strong independence properties of Poisson processes, it can be shown that

$$
\Theta_{k}(\mathcal{A})=\frac{1}{k !} \int_{\mathcal{E}_{n-1}^{n}} \cdots \int_{\mathcal{E}_{n-1}^{n}} \mathbf{1}_{\mathcal{A}}\left(H_{1} \cap \cdots \cap H_{k}\right) \Theta\left(\mathrm{d} H_{1}\right) \cdots \Theta\left(\mathrm{d} H_{k}\right)
$$

for $\mathcal{A} \in \mathcal{B}\left(\mathcal{E}_{n-k}^{n}\right)$. This formula is similar to (90). From (97) and the regularity of $X$ it can be deduced that $X_{k}$ is also regular. In particular, the intensity function of $X_{k}$ is defined; we denote it by $\gamma_{k}$. A computation gives

$$
\gamma_{k}(x)=\frac{1}{k !} \int_{S^{n-1}} \cdots \int_{S^{n-1}}\left[u_{1}, \ldots, u_{k}\right] \rho_{x}\left(\mathrm{~d} u_{1}\right) \cdots \rho_{x}\left(\mathrm{~d} u_{k}\right) .
$$

The geometric question we want to answer is whether one can find sharp bounds for the intensity function of the intersection process of order $k$ in terms of the intensity function of the process $X$ itself. The answer comes from a beautiful interpretation of the integral (98). We have mentioned in Subsection 4.1 that the integral representation (84) implies the representation (85) for the $k$-dimensional projection volume. Further, we know from (17) that averaging the $k$-dimensional projection volumes over all directions gives the $k$ th intrinsic volume. Applying this to the convex body $\Pi_{x}$, which has the representation (96), we must get a formula for the intrinsic volume $V_{k}\left(\Pi_{x}\right)$. The result is

$$
V_{k}\left(\Pi_{x}\right)=\frac{2^{k}}{k !} \int_{S^{n-1}} \cdots \int_{S^{n-1}}\left[u_{1}, \ldots, u_{k}\right] \rho_{x}\left(\mathrm{~d} u_{1}\right) \cdots \rho_{x}\left(\mathrm{~d} u_{k}\right) .
$$

Comparison with (98) now shows that

$$
\gamma_{k}(x)=V_{k}\left(2^{-1} \Pi_{x}\right) .
$$

The intrinsic volumes of convex bodies satisfy well-known inequalities. As a consequence, and with some additional arguments concerning the equality case, the following sharp estimate can be obtained.

Theorem 4.7. Let $X$ be a regular Poisson hyperplane process in $\mathbb{R}^{n}$ with intensity function $\gamma$. Let $k \in\{2, \ldots, n\}$, and let $\gamma_{k}$ be the intensity function of the intersection process $X_{k}$ of order $k$. Then 


$$
\gamma_{k}(x) \leq \frac{\left(\begin{array}{l}
n \\
k
\end{array}\right) \kappa_{n-1}^{k}}{n^{k} \kappa_{n-k} \kappa_{n}^{k-1}} \gamma(x)^{k}
$$

for $x \in \mathbb{R}^{n}$. Equality for all $x \in \mathbb{R}^{n}$ holds if and only if the process $X$ is stationary and isotropic.

Hints to the literature. Processes of $k$-flats are treated, for example, in [52]. The contents of this subsection are taken from [49], where full proofs can be found.

\section{References}

1. Alexander, R.: Zonoid theory and Hilbert's fourth problem. Geom. Dedicata $\mathbf{2 8}$ (1988), 199-211.

2. Álvarez Paiva, J.C., Fernandes, E.: Crofton formulas in projective Finsler spaces. Electronic Res. Announcements AMS 4 (1998), 91-100.

3. Federer, H.: Curvature measures. Trans. Amer. Math. Soc. 93 (1959), 418-491.

4. Giger, H., Hadwiger, H.: Über Treffzahlwahrscheinlichkeiten im Eikörperfeld. $Z$. Wahrscheinlichkeitsth. verw. Geb. 10 (1968), 329-334.

5. Glasauer, S.: Integralgeometrie konvexer Körper im sphärischen Raum. Doctoral dissertation, Universität Freiburg i. Br., 1995.

6. Glasauer, S.: A generalization of intersection formulae of integral geometry. Geom. Dedicata 68 (1997), 101-121.

7. Glasauer, S.: Kinematic formulae for support measures of convex bodies. Beitr. Algebra Geom. 40 (1999), 113-124.

8. Goodey, P., Weil, W.: Representations of mixed measures with applications to Boolean models. Rend. Circ. Mat. Palermo, Ser. II, Suppl. 70 (2002), vol. I, $325-346$.

9. Goodey, P., Weil, W.: Translative and kinematic integral formulae for support functions II. Geom. Dedicata 99 (2003), 103-125.

10. Groemer, H.: On the extension of additive functionals on classes of convex sets. Pacific J. Math. 75 (1978), 397-410.

11. Gruber, P., Schneider, R.: Problems in geometric convexity. In J. Tölke and J.M. Wills (Eds.), Contributions to Geometry, pp. 255-278, Birkhäuser, Basel, 1979.

12. Hadwiger, H: Einige Anwendungen eines Funktionalsatzes für konvexe Körper in der räumlichen Integralgeometrie. Monatsh. Math. 54 (1950), 345-353.

13. Hadwiger, H: Beweis eines Funktionalsatzes für konvexe Körper. Abh. Math. Sem. Univ. Hamburg 17 (1951), 69-76.

14. Hadwiger, H: Additive Funktionale k-dimensionaler Eikörper. I. Arch. Math. 3 (1952), 470-478.

15. Hadwiger, H: Integralsätze im Konvexring. Abh. Math. Sem. Univ. Hamburg 20 (1956), 136-154.

16. Hadwiger, H: Vorlesungen über Inhalt, Oberfläche und Isoperimetrie. Springer, Berlin, 1957.

17. Hug, D.: Measures, curvatures and currents in convex geometry. Habilitationsschrift, Universität Freiburg i. Br., 1999 
18. Hug, D., Last, G.: On support measures in Minkowski spaces and contact distributions in stochastic geometry. Ann. Prob. 28 (2000), 796-850.

19. Hug, D., Last, G., Weil, W.: A local Steiner-type formula for general closed sets and applications. Math. Z. 246 (2004), 237-272.

20. Hug, D., Mani-Levitska, P., Schätzle, R.: Almost transversal intersections of convex surfaces and translative integral formulae. Math. Nachr. 246-247 (2002), 121-155.

21. Hug, D., Schätzle, R.: Intersections and translative integral formulas for boundaries of convex bodies. Math. Nachr. 226 (2001), 99-128.

22. Hug, D., Schneider, R.: Kinematic and Crofton formulae of integral geometry: recent variants and extensions. In Homenatge al professor Lluís Santaló $i$ Sors (C. Barceló i Vidal, ed.), Universitat de Girona, 2002, pp. 51-80.

23. Kiderlen, M.: Schnittmittelungen und äquivariante Endomorphismen konvexer Körper. Doctoral dissertation, Universität Karlsruhe, 1999.

24. Klain, D.A.: A short proof of Hadwiger's characterization theorem. Mathematika 42 (1995), 329-339.

25. Klain, D.A., Rota, G.-C.: Introduction to Geometric Probability. Cambridge University Press, Cambridge, 1997.

26. McMullen, P.: Mixed fibre polytopes. Preprint (2003).

27. Pogorelov, A.V.: Hilbert's Fourth Problem. Scripta Series in Mathematics. V.H. Winston \& Sons, Washington, D.C.; A Halstead Press Book, John Wiley \& Sons, New York 1979 (Russian Original: Izdat. "Nauka", Moscow, 1974).

28. Rataj, J.: The iterated version of a translative integral formula for sets of positive reach. Rend. Circ. Mat. Palermo, Ser. II, Suppl., 46 (1997), 129-138.

29. Rataj, J.: Remarks on a translative formula for sets of positive reach. Geom. Dedicata 65 (1997), 59-62.

30. Rataj, J.: Translative and kinematic formulae for curvature measures of flat sections. Math. Nachr. 197 (1999), 89-101.

31. Rataj, J.: Absolute curvature measures for unions of sets with positive reach. Preprint 00/22, Universität Karlsruhe (2000)

32. Rataj, J.: A translative integral formula for absolute curvature measures. Geom. Dedicata 84 (2001), 245-252.

33. Rataj, J., Zähle, M.: Mixed curvature measures for sets of positive reach and a translative integral formula. Geom. Dedicata 57 (1995), 259-283.

34. Rataj, J., Zähle, M.: Curvatures and currents for unions of sets with positive reach, II. Ann. Global Anal. Geom. 20 (2001), 1-21.

35. Rataj, J., Zähle, M.: A remark on mixed curvature measures of sets with positive reach. Beitr. Algebra Geom. 43 (2002), 171-179.

36. Rother, W., Zähle, M.: A short proof of a principal kinematic formula and extensions. Trans. Amer. Math. Soc. 321 (1990), 547-558.

37. Santaló, L.A.: Integral Geometry and Geometric Probability. Encyclopedia of Mathematics and Its Applications, vol. 1. Addison-Wesley, Reading, MA, 1976.

38. Schmidt, V., Spodarev, E.: Joint estimators for the specific intrinsic volumes of stationary random sets. Preprint (2003).

39. Schneider, R.: Convex Bodies: the Brunn-Minkowski Theory. Encyclopedia of Mathematics and Its Applications 44, Cambridge University Press, Cambridge, 1993.

40. Schneider, R.: An extension of the principal kinematic formula of integral geometry. Rend. Circ. Mat. Palermo, Ser. II, Suppl., 35 (1994), 275-290. 
41. Schneider, R: On areas and integral geometry in Minkowski spaces. Beitr. Algebra Geom. 38 (1997), 73-86.

42. Schneider, R.: Convex bodies in exceptional relative positions. J. London Math. Soc. 60 (1999), 617-629.

43. Schneider, R.: Mixed functionals of convex bodies. Discrete Comput. Geom. 24 (2000), 527-538.

44. Schneider, R.: On the Busemann area in Minkowski spaces. Beitr. Algebra Geom. 42 (2001), 263-273.

45. Schneider, R.: Crofton formulas in hypermetric projective Finsler spaces. Arch. Math. 77 (2001), 85-97.

46. Schneider, R.: On integral geometry in projective Finsler spaces. Izvestija NAN Armenii. Matematika 37 (2002), 34-51.

47. Schneider, R.: Mixed polytopes. Discrete Comput. Geom. 29 (2003), 575-593.

48. Schneider, R.: An integral geometric theorem for simple valuations. Beitr. Algebra Geom. 44 (2003), 487-492.

49. Schneider, R.: Nonstationary Poisson hyperplanes and their induced tessellations. Adv. Appl. Probab. (SGSA) 35 (2003), 139-158.

50. Schneider, R., Weil, W.: Translative and kinematic integral formulas for curvature measures. Math. Nachr. 129 (1986), 67-80.

51. Schneider, R., Weil, W.: Integralgeometrie. Teubner, Stuttgart, 1992.

52. Schneider, R., Weil, W.: Stochastische Geometrie. Teubner, Stuttgart, 2000.

53. Schneider, R., Wieacker, J.A.: Integral geometry in Minkowski spaces. Adv. Math. 129 (1997), 222-260.

54. Szabó, Z.I.: Hilbert's Fourth Problem, I. Adv. Math. 59 (1986), 185-301.

55. Weil, W.: Kontinuierliche Linearkombination von Strecken. Math. Z. 148 (1976), 71-84.

56. Weil, W.: Centrally symmetric convex bodies and distributions, II. Israel J. Math. 32 (1979), 173-182.

57. Weil, W.: Iterations of translative integral formulae and non-isotropic Poisson processes of particles. Math. Z. 205 (1990), 531-549.

58. Weil, W.: Translative and kinematic integral formulae for support functions. Geom. Dedicata 57 (1995), 91-103.

59. Weil, W.: Mixed measures and functionals of translative integral geometry. Math. Nachr. 223 (2001), 161-184.

60. Zähle, M.: Integral and current representations of Federer's curvature measures. Arch. Math. 46 (1986), 557-567.

61. Zähle, M.: Curvatures and currents of sets with positive reach. Geom. Dedicata 23 (1987), 155-171.

62. Zähle, M.: Nonosculating sets of positive reach. Geom. Dedicata 76 (1999), $183-187$. 